\title{
Primary prevention of coronary heart disease: integration of new data, evolving views, revised goals, and role of rosuvastatin in management. A comprehensive survey
}

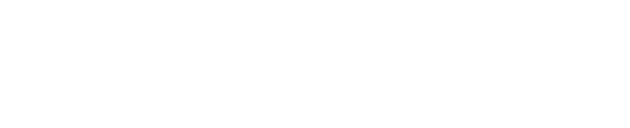

\section{Richard Kones}

The Cardiometabolic Research Institute, Houston, TX, USA
Correspondence: Richard Kones Cardiometabolic Research Institute, 8I8I Fannin Street, U3|4, Houston, TX 77054, USA

$\mathrm{Tel}+\mathrm{I} 7137909100$

Fax +17137909292

Email drrkones@comcast.net

\begin{abstract}
A recent explosion in the amount of cardiovascular risk and incipient, undetected subclinical cardiovascular pathology has swept across the globe. Nearly $70 \%$ of adult Americans are overweight or obese; the prevalence of visceral obesity stands at $53 \%$ and continues to rise. At any one time, $55 \%$ of the population is on a weight-loss diet, and almost all fail. Fewer than $15 \%$ of adults or children exercise sufficiently, and over $60 \%$ engage in no vigorous activity. Among adults, $11 \%-13 \%$ have diabetes, $34 \%$ have hypertension, $36 \%$ have prehypertension, $36 \%$ have prediabetes, $12 \%$ have both prediabetes and prehypertension, and $15 \%$ of the population with either diabetes, hypertension, or dyslipidemia are undiagnosed. About one-third of the adult population, and $80 \%$ of the obese, have fatty livers. With $34 \%$ of children overweight or obese, prevalence having doubled in just a few years, type 2 diabetes, hypertension, dyslipidemia, and fatty livers in children are at their highest levels ever. Half of adults have at least one cardiovascular risk factor. Not even $1 \%$ of the population attains ideal cardiovascular health. Despite falling coronary death rates for decades, coronary heart disease (CHD) death rates in US women 35 to 54 years of age may now be increasing because of the obesity epidemic. Up to $65 \%$ of patients do not have their conventional risk biomarkers under control. Only $30 \%$ of high risk patients with CHD achieve aggressive low density lipoprotein (LDL) targets. Of those patients with multiple risk factors, fewer than $10 \%$ have all of them adequately controlled. Even when patients are titrated to evidence-based targets, about $70 \%$ of cardiac events remain unaddressed. Undertreatment is also common. About two-thirds of high risk primary care patients are not taking needed medications for dyslipidemia. Poor patient adherence, typically below $50 \%$, adds further difficulty. Hence, after all such fractional reductions are multiplied, only a modest portion of total cardiovascular risk burden is actually being eliminated, and the full potential of risk reduction remains unrealized. Worldwide the situation is similar, with the prevalence of metabolic syndrome approaching 50\%. Primordial prevention, resulting from healthful lifestyle habits that do not permit the appearance of risk factors, is the preferred method to lower cardiovascular risk. Lowering the prevalence of obesity is the most urgent matter, and is pleiotropic since it affects blood pressure, lipid profiles, glucose metabolism, inflammation, and atherothrombotic disease progression. Physical activity also improves several risk factors, with the additional potential to lower heart rate. Given the current obstacles, success of primordial prevention remains uncertain. At the same time, the consequences of delay and inaction will inevitably be disastrous, and the sense of urgency mounts. Since most CHD events arise in a large subpopulation of low- to moderate-risk individuals, identifying a high proportion of those who will go on to develop events with accuracy remains unlikely. Without a refinement in risk prediction, the current model of targeting high-risk individuals for aggressive therapy may not
\end{abstract}


succeed alone, especially given the rising burden of risk. Estimating cardiovascular risk over a period of 10 years, using scoring systems such as Framingham or SCORE, continues to enjoy widespread use and is recommended for all adults. Limitations in the former have been of concern, including the under- or over-estimation of risk in specific populations, a relatively short 10-year risk horizon, focus on myocardial infarction and CHD death, and exclusion of family history. Classification errors may occur in up to $37 \%$ of individuals, particularly women and the young. Several different scoring systems are discussed in this review. The use of lifetime risk is an important conceptual advance, since $\geq 90 \%$ of young adults with a low 10 -year risk have a lifetime risk of $\geq 39 \%$; over half of all American adults have a low 10-year risk but a high lifetime risk. At age 50 the absence of traditional risk factors is associated with extremely low lifetime risk and significantly greater longevity. Pathological and epidemiological data confirm that atherosclerosis begins in early childhood, and advances seamlessly and inexorably throughout life. Risk factors in childhood are similar to those in adults, and track between stages of life. When indicated, aggressive treatment should begin at the earliest indication, and be continued for years. For those patients at intermediate risk according to global risk scores, C-reactive protein (CRP), coronary artery calcium (CAC), and carotid intima-media thickness (CIMT) are available for further stratification. Using statins for primary prevention is recommended by guidelines, is prevalent, but remains underprescribed. Statin drugs are unrivaled, evidence-based, major weapons to lower cardiovascular risk. Even when low density lipoprotein cholesterol (LDL-C) targets are attained, over half of patients continue to have disease progression and clinical events. This residual risk is of great concern, and multiple sources of remaining risk exist. Though clinical evidence is incomplete, altering or raising the blood high density lipoprotein cholesterol (HDL-C) level continues to be pursued. Of all agents available, rosuvastatin produces the greatest reduction in LDL-C, LDL-P, and improvement in apoA-I/apoB, together with a favorable safety profile. Several recent proposals and methods to lower cardiovascular risk are reviewed. A combination of approaches, such as the addition of lifetime risk, refinement of risk prediction, guideline compliance, novel treatments, improvement in adherence, and primordial prevention, including environmental and social intervention, will be necessary to lower the present high risk burden.

Keywords: primary prevention, cardiovascular risk, coronary heart disease, primordial prevention, rosuvastatin, JUPITER study, statin drugs, C-reactive protein, inflammation, low-density lipoprotein, high-density lipoprotein, diabetes, metabolic syndrome, Framingham risk score, Reynolds risk score, SCORE, coronary artery calcification, carotid intima-media thickness, hypertension, obesity, non-HDLcholesterol, LDL-P, dysfunctional HDL, lifetime risk, advanced lipid testing, Bogalusa Heart Study

\section{Introduction: the enormity of the challenge}

Cardiovascular disease (CVD) claims 2,300 lives each day in the United States, averaging one death every 39 seconds. In 2006, 81 million people in America suffered with $\geq$ one type of cardiovascular disease. Presently one in three Americans has CVD, and 35\% of all American deaths are due to the scourge. ${ }^{1}$ Coronary heart disease (CHD) is the leading cause of death in the US and the UK: one coronary event occurs every 25 seconds in America, with $34 \%$ dying within the same year, amounting to one death every minute. On an annual basis, about 785,000 Americans have new coronary attacks, and 470,000 have a recurrence, with an estimated 195,000 first myocardial infarctions that occur silently. ${ }^{1}$ There were about 406,351 deaths in the United States from CHD in 2007, approximately one in six of all deaths. ${ }^{2}$ Thirty-five years ago, the number of coronary deaths was significantly higher, and the prognosis was limited by the relative dearth of technical, invasive, and pharmacological options. ${ }^{3}$ Currently, there are about 6.2 million hospitalizations for cardiovascular disease each year in the USA, 730,000 hospitalizations for stroke, and 7.2 million cardiac and vascular procedures are performed. ${ }^{4}$ A similar situation now exists world-wide. ${ }^{5,6}$
CVD now consumes $17 \%$ of the national health budget in America, where health expenditures, matching the body mass indices (BMIs), are the highest in the world. The cost of CVD in the USA has recently grown at an average annual rate of $6 \%$, and accounts for about $15 \%$ of the rise in total medical expenditures. During the next 20 years, the prevalence of CVD will rise by about $10 \%$, and the costs attributable to CVD will triple, simply because of demographic changes in the population. By 2030, without any change in prevention or treatment practices, it is projected that the number of people with one of more forms of heart disease will increase from $36.9 \%$ to $40.5 \%$, to total 116 million American adults. By then, the incidence of heart failure and stroke will have each risen by $\approx 25 \%$. ${ }^{7}$

Although the death rate from CHD has fallen, the prevalence of risk factors, especially obesity and diabetes, continues at an alarming level, actually making the above estimates conservative. The present crop of adolescents who are overweight will increase the number of obese adults an additional $5 \%-15 \%$ by 2035 , generating another 100,000 prevalent cases of CHD. There is increasing evidence that obesity is offsetting improvements in CHD mortality, ${ }^{8,9}$ and a recent comprehensive review by the National Research Council found that the high prevalence of risk factors, particularly obesity, was a major factor accounting for 
lagging improvement in longevity in America and developed countries. ${ }^{10}$

Despite remarkable advances considered science fiction just a short time ago, treatment success, as cardiologists well know, remains a constant uphill struggle. The sobering figures above have prompted many epidemiologists and preventive cardiologists to suggest that changes in thinking and approach in primary prevention have been needed for some time. In contrast with the dire predictions outlined above, another pathway is possible. A rich data base, ${ }^{11}$ including the INTERHEART study ${ }^{12}$ and recent models, ${ }^{13}$ suggest a refreshing, alternative direction. By lowering the prevalence of risk factors, there will be an anticipated reduction in both CVD events and cardiovascular mortality of striking proportions. ${ }^{7}$

Most risk factors that drive cardiovascular disease have genetic, physiologic, behavioral, and environmental components. Nonmodifiable risk factors include age, genetics, and gender. Modifiable risk factors comprise smoking, dyslipidemia, hypertension, and diabetes, with obesity and metabolic syndrome usually included. Emerging, "novel," or nontraditional risk factors for risk assessment include C-reactive protein (CRP), lipoprotein-associated phospholipase $\mathrm{A}_{2}$ (Lp-PLA 2$),{ }^{14}$ LDL particle number (LDL-P), fibrinogen, lipoprotein (a) [Lp(a)], small, dense LDL, triglycerides (TG) and triglyceride-enriched particles, plasminogen activator inhibitor (PAI-1), interleukin-6 (IL-6), and others. Leading contenders among imaging techniques capable of refining risk prediction or improving management include measurement of carotid intima-media thickness (CIMT), coronary artery calcification (CAC), and MRI or CT coronary angiography (Table 1).

Risk factors influence the pathobiology of atherosclerosis and coronary artery disease in a continuum during a complex interaction of genetics and environmental factors throughout life. Modifying events along this long time span include lifestyle changes, primarily nutrition, exercise, stress, comorbidities, pharmaceuticals, particularly statins, percutaneous interventions, and surgery.

A significant number of pharmacological agents are now prescribed for cardiovascular risk reduction during both primary and secondary prevention, as well as during treatment of coronary heart disease (CHD). A recent paper focused on rosuvastatin, reviewing its properties and some details about use in primary prevention. ${ }^{15}$ This complementary paper examines new data, changes in views about primary prevention, the rationale for alternative approaches, and surveys several recent proposals to improve risk stratification and clinical outcomes.
Table I Common tools used in cardiovascular risk assessment

Clinical evaluation: history and physical examination

Family history

Global Risk Scoring (FRS, SCORE, Reynolds)

Calculation of lifetime risk

Electrocardiogram (ECG)

Echocardiography

Stress (Exercise) electrocardiography

Myocardial perfusion imaging (MPI)

Stress (Exercise) echocardiography

LDL-C (apolipoprotein B, non-HDL-C)

HDL-C

Lipoprotein "little a" $(\operatorname{Lp}(\mathrm{a}))$

Lipoprotein fractionation (VAP, NMR)

hsC-reactive protein (CRP)

Lipoprotein-associated phospholipase A2 (Lp-PLA2)

Coronary angiography

Carotid intima-media thickness using ultrasound (CIMT)

Computed tomography for coronary calcium (CAC)

CT coronary angiography

MRI coronary angiography

Abbreviations: FRS, Framingham Risk Score; LDL-C, low density lipoprotein cholesterol; HDL-C, high density lipoprotein cholesterol; CT, computerized tomography; MRI, magnetic resonance imaging.

\section{Types of prevention}

Prevention, although relatively unfashionable when compared to cutting-edge high-tech procedures, addresses two of the three goals of medicine: preventing disease, relief of suffering, and prolonging life. On the other hand, percutaneous coronary intervention (PCI) and coronary artery bypass grafting (CABG) excel in relieving suffering unresponsive to medical therapy, making the combination of prevention and therapeutic procedures a complementary union of partners in a continuum of management.

Prevention may refer generally to screening and immunizations to detect, forestall, or limit serious disease. Primary prevention denotes delaying or limiting a first event in individuals who have not yet been formally diagnosed with heart disease. Usage includes individuals with risk factors, the latter commonly regarded as if they were in fact diseases themselves, since often they are simultaneously causes, surrogates, and targets for therapy. Secondary prevention seeks to prevent, postpone, or limit recurrence of a clinical event in patients who have been previously diagnosed with cardiovascular disease. The functional distinction between the types of prevention appears to be blurring. ${ }^{16}$ For complex reasons, prevention receives $\leq 3 \%$ of the health care budget, ${ }^{17}$ and prevention measures are underemployed, even in the context of a current $\approx 55 \%$ utilization of all recommended effective therapies. ${ }^{18}$ Cost-ineffectiveness has been disproved for individual primary prevention techniques. ${ }^{19,20}$ The 
National Institute for Health and Clinical Excellence (NICE) in the UK estimated that a $1 \%$ population-wide lowering of cardiac risk factors would net substantial savings, ${ }^{21}$ yet even with widespread agreement, prevention services remain low on US payers' priorities, and are similarly undervalued by the public.

\section{Primordial prevention}

Primordial prevention, a term first used by Strasser, ${ }^{22}$ refers to individual behavioral lifestyle characteristics that achieve a level of health that does not permit risk factors to appear. The American Heart Association (AHA) incorporated the powerful principle of primordial prevention in defining "ideal cardiovascular health" as a goal in reducing cardiac and stroke mortality $20 \%$ by year $2020 .^{2}$ Ideal cardiovascular health consists of the absence of cardiovascular disease, a healthy lifestyle (sufficient exercise, a superior diet score, absence of smoking, and BMI $<25 \mathrm{~kg} / \mathrm{m}^{2}$ ), and ideal health factors (untreated normal values of blood pressure, cholesterol, and fasting glucose). These parameters, termed Life's Simple $7^{\mathrm{TM}}$, are presented in an AHA educational site, mylifecheck.org, which promotes primordial prevention (Table 2). Presentation of material is layered, clear, in language that is easily understood, and user-friendly. The AHA Goals also emphasized the need for a plant-based diet containing legumes, nuts, seeds, and minimum amounts of trans and saturated fats. ${ }^{2}$

Reversal of existing risk factors does not equal the degree of protection afforded by primordial prevention..$^{23,24}$ Since atherosclerosis begins at an early age and progresses

Table 2 Ideal Cardiovascular Health, defined by the American Heart Association, now known as "Life's Simple 7 ${ }^{\text {TM", }}$ is composed of four behaviors and three factors ${ }^{2}$,*

I. Not smoking or quitting over I year ago.

2. A body mass index $<25 \mathrm{~kg} / \mathrm{m}^{2}$.

3. Exercising at a moderate intensity $\geq 150$ minutes (or 75 minutes at vigorous intensity) each week.

4. Eating a "healthy diet": adhering to four to five important dietary components

- sodium intake < I.5 g/day;

- sugar-sweetened beverage intake $<36$ oz weekly;

$-\geq 4.5$ cups of fruits and vegetables/day;

- $\geq$ three I oz servings of fiber-rich whole grains/day;

$-\geq$ two 3.5 oz servings of oily fish/week.

5. Maintaining total cholesterol (TC) $<200 \mathrm{mg} / \mathrm{dL}$.

6. Keeping blood pressure $<120 / 80 \mathrm{mmHg}$.

7. Keeping fasting blood glucose $<100 \mathrm{mg} / \mathrm{dL}$.

Notes: *Other recommendations include $\geq$ four servings of nuts, legumes and seeds/week; $\leq$ less servings of processed meats/week; less than $7 \%$ total energy intake as saturated fat. throughout life, primordial prevention is uniquely successful in combating CHD, but also adds protection against other degenerative diseases to reduce all-cause mortality. Dietary changes alone can produce - and completely reverse - lesions of CHD in primates ${ }^{25}$ and intensive lifestyle programs may cause lesion regression in humans. ${ }^{26}$ Individuals who achieve primordial prevention are uncommon, with fewer than 5\% achieving ideal cardiovascular health as they approach middle life. ${ }^{2}$ When individuals do enter middle age without risk factors, their cardiovascular protection is extraordinary and they enjoy an additional 10 years of life. ${ }^{27,28} \mathrm{~A}$ number of recent impressive prospective studies and models support these findings, suggesting that population-based primordial prevention is capable of reducing CHD deaths to approximately $10 \%$ of the current expected rate. ${ }^{8,11}$

Among the strategies to lower risk are proposals involving reeducation, reorientation, and motivation of individuals so their lifestyles do not permit risk factors to develop, or slow to the extent that their lifetime risk is lowered. Weight loss of $15 \%$ or more of body weight with other dietary and lifestyle interventions, especially exercise, will drastically reduce cardiovascular risk up to $45 \%$ and simultaneously lower CRP levels. ${ }^{29}$ School- and community-based initiatives, improvement in diet and food quality, social support, and other incentives are among the approaches available. Health, nutrition, and exercise illiteracy are prevalent, with misinformation and unrealistic expectations also common. From an intuitive and qualitative view, therefore, corrective education would appear beneficial. Overall, however, such current methods to positively influence individual behavior have been unsuccessful. A recent Cochrane review of 55 trials reported that counseling and educational programs can lower risk factor burdens modestly, but do not lower CHD events, total or CHD mortality in the general population, and are therefore not recommended or evidence-based. ${ }^{30}$ They did find, however, that patient education and counseling may be useful in high-risk diabetic or hypertensive populations, from which the inference was made that patients with pre-existing disease benefited, but not those who were undiagnosed. Removal of education, behavioral intervention and counseling, as suggested, would leave policy decisions, largely environmental, as the remaining technique available for primordial prevention.

This report ${ }^{30}$ uniquely disagrees with a large body of evidence that preceeded it, and is at odds with pathological data and available guidelines concerning cardiovascular risk management. ${ }^{2,6,31,32}$ It suggests that current initiatives, such as the American Heart Association's Life's Simple $7^{\mathrm{TM}}$, will 
have limited effect, and that public health policies to lower cardiovascular risk in most countries may be misguided. Ignoring patient education, as is implied, is also contrary to the Institute of Medicine's goal of a patient-centered health system $^{33}$ and simultaneously undermines a core constituent of models to improve care and patient value by allowing participation in clinical decision-making. ${ }^{34}$ The components of patient attitudes and behavior are complex, and are of such crucial importance that further analyses, such as done by White, ${ }^{35}$ are needed to refine future approaches.

The accompanying editorial ${ }^{36}$ observed that there were no recent large-scale randomized controlled trials on the issue, and reviewed the pitfalls and perils involved in making educational and behavioral modification programs effective. The design, recruitment, administration (especially adherence verification), and successful completion of such a study presents enormous challenges. Funding for such a project is highly unlikely. In the absence of such randomized trials, the editorialist stressed reducing dietary salt intake, legislating for smoke-free public spaces, and exercise parks to facilitate primordial prevention. Unfortunately, these policies too have encountered obstacles. Environmental restructuring to improve health has been challenged legally, and has been considered intrusive in some venues.

Shortly thereafter, the Global Burden of Metabolic Risk Factors of Chronic Diseases Collaborating Group published three reports that described global population-level changes in body-mass index (BMI), systolic blood pressure, and total cholesterol over three decades. ${ }^{37-39}$ Involving 3.0-9.1 million participants over 321-960 country-years, their three studies found that obesity rates doubled between 1980 and 2008, overweight affected one in three adults, and obesity affected one in nine adults in the world. Global BMI increased on average 0.4 to 0.5 kilograms, about 0.9 to 1.1 pounds, per decade for men and women. Changes in blood pressure and cholesterol varied according to income and location, and sometimes were disparate with the rises in BMIs. Even so, the high cardiovascular disease burden in low- and middleincome countries over the next few decades was deemed dismal and an emergency. ${ }^{40}$ The solution proposed to avert tens of millions of preventable deaths was, not surprisingly, population-based risk control.

\section{High-risk individual model vs a population-based model}

The current paradigm in both primary and secondary prevention seeks to identify individuals at high risk for cardiovascular events through screens and target them for additional evaluation, stratification, and intensive treatment. Guidelines concentrate on maximizing use of these steps to produce the greatest reduction in clinical events, quality of life, and survival. Throughout the past four decades, this approach has been rewarding, with extraordinary advances that have been reflected in large decreases in cardiovascular mortality. For example, for every $10 \%$ rise in treatments of LDL-C elevations in the population younger than 80 years, approximately 8,000 deaths could be prevented annually, ${ }^{20}$ and for a $10 \%$ rise in hypertension therapy, about 14,000 deaths per annum would be averted. A fall in risk factors within the population accounts for about half of the lowering of death rates during the past 40 years. ${ }^{41-45}$ Despite this improvement, death from coronary heart disease remains at the top of the list, and will also be the leading cause of death world-wide within the decade. Moreover, the recent lowering of CHD deaths has shown signs of slowing, probably due to the dual epidemics of obesity and diabetes. From a public health perspective, somewhat over $40 \%$ of CHD events occur in the $6 \%$ of the population with manifest disease, much of which is preventable with effective measures. ${ }^{46}$

Geoffrey Rose ${ }^{47-50}$ a British epidemiologist, compared health consequences when individual care is given to highrisk individuals, often those who make poor lifestyle choices, compared to treating the entire population. He also addressed environmental and behavior characteristics of the population in general. Following a population-based strategy to control risk, changes in a large number of people resulted in a smaller individual benefit, and required a relatively larger political and economic change for success. ${ }^{47}$ Cultural and regional differences, ie, "diversity", complicated implementation. Rose said "mass diseases and mass exposures require mass remedies", emphasizing that sick individuals arise from sick populations, and that the number of persons with undesirable levels of risk factors depends upon the average risk in the population. ${ }^{49} \mathrm{He}$ observed that the behaviors of many subjects with low risk have a greater impact upon disease prevalence than fewer individuals with high risk. ${ }^{48}$ Treatment of high-risk individuals tended to be crisis intervention and only palliative, whereas population intervention targeted fundamental causes.

Less than $10 \%$ of the population is at high risk for cardiovascular disease, but the low to intermediate risk group is so large that most cardiovascular events will actually arise from that population. ${ }^{1,47,51}$ As a result, $90 \%$ or more of cardiovascular events will occur in individuals with one or more risk factor, somewhat under half of the population. ${ }^{52}$ Importantly, this subpopulation with cardiac 
events would not qualify for intensive diagnosis or treatment using high-risk filters. Lowering the risk factor burden in the general population through lifestyle, environmental, and social changes would be expected to produce greater reductions in CHD risk.

\section{High burden of cardiovascular risk Past and future trends are unfavorable}

During the last two decades, adherence to a healthy lifestyle has deteriorated significantly. ${ }^{11,53,54}$ From 1988-2006, the prevalence of obesity increased from $28 \%$ to $36 \%$, consumption of $\geq 5$ portions of vegetables or fruits was nearly halved, level of physical activity also fell, while alcohol consumption rose. Patients with diabetes or heart disease also reported poor performance in these categories.

Examining 10-year trends in chronic illnesses, Paez and collaborators ${ }^{55}$ used the prevalence of self-reported conditions during expenditure surveys, and noted that in $2005,44 \%$ of Americans had at least one chronic disease. The number of persons with three chronic conditions rose sharply, however, when compared with the number with one illness. For persons ages 45-64 years, the prevalence of multiple chronic conditions in 1996 was 13\%, and by 2005 rose to $22 \%$. In 2010, an analysis by Health and Human Services reported $27 \%$ of the population suffered with multiple chronic illnesses. ${ }^{55}$

Today very few Americans are at low risk for heart disease. Continuing reports of rising prevalence of overweight and obesity not only reflect acceleration, but absolute levels are higher than ever before. ${ }^{56,57}$ Since the data are self-reported, the actual numbers are likely worse. Even in the face of consistently rising risk, $65 \%$ of Americans rate their health as good, but overweight and obesity alone exceed this number. Over $55 \%$ of young adults have at least one CVD risk factor, and over $37 \%$ reported having $\geq$ two risk factors for CVD (with only $35 \%$ under control), all reflecting a dissociation between perception and reality. Indeed, the US Centers for Disease Control and Prevention (CDC) reports that Americans are now sicker than ever. ${ }^{58}$ In Canada too, $90 \%$ of citizens report they are healthy, when actually $90 \%$ of them have $\geq$ one risk factor, also an apparent denial of the prevalence of risk factors. ${ }^{59}$

According to American Heart Association criteria, perhaps 5\% of Americans were considered to have ideal cardiovascular health when the 2010 Impact Goals were presented, ${ }^{2}$ but subsequent evaluation reveals it is actually less than $1 \%$. If recent increases in the incidence of diabetes continue, the prevalence of diabetes in American adults, including undiagnosed cases, is projected to rise from the present $14 \%$ to $33 \%$ of American adults by $2050 .{ }^{60}$ Even with vigorous intervention, a significant increase in diabetes prevalence will be inevitable. ${ }^{61}$ Clyde Yancy MD, Chief of Cardiology at Northwestern University and past president of the AHA, in an editorial about the challenges of lowering risk in the population, recently termed the cardiovascular health status in America "miserable". ${ }^{62}$ Unfortunately, an end to the dual epidemics of obesity and diabetes is not in sight. ${ }^{63}$ Although excellent information is available, ${ }^{64,65}$ there is no comprehensive, population-wide plan being implemented with any evidenced-based hope of reversing the rise in prevalence for either condition. ${ }^{58,63}$

\section{Prevalence of risk factors is high}

The current cardiovascular risk burden is oppressively high. According to recent NHANES data, about half of American adults have one or more of the three major risk factors: dyslipidemia, hypertension, or diabetes. ${ }^{53}$ Approximately $13 \%$ have two of these risk factors, and about 3\% have all three. The National Center for Health Statistics also estimates that $15 \%$ of adults have one of more of the three risk factors but remain undiagnosed and hence untreated. The 2010 Update on Heart Disease and Stroke Statistics ${ }^{1}$ reported the following prevalence rates from various sources: $34 \%$ hypertension, $33 \%$ obesity, $32 \%$ pediatric obesity or overweight, 29\% prediabetes, $8 \%$ undiagnosed diabetes, $34 \%$ metabolic syndrome, $21.2 \%$ tobacco use, and 59\% abstaining from any vigorous activity. Other reports, a short interval later, indicate a higher incidence of hypertension, $36 \%$ prediabetes, and $27 \%$ undiagnosed diabetes, and $11.2 \%$ of adults with both prehypertension and prediabetes. ${ }^{2}$ In 2009, the diabetes population was predicted to double or triple within 25 years. ${ }^{60}$ During the same period, a review of surveys estimated that among Americans $\geq 30$ years, $13.7 \%$ of men and $11.9 \%$ of women had diagnosed diabetes, and about one-third of them had not yet been diagnosed. The most recent data from the $\mathrm{CDC}^{66}$ amended these figures, reporting a rise in prevalence of prediabetes to $35 \%$ in adults older than 20 years, to $50 \%$ in those over 65 years of age. The strain on the healthcare system resulting from both obesity ${ }^{67}$ and diabetes is remarkably high. ${ }^{60,68}$ Since the surveys incorporate older information, self-reporting, and imperfect criteria in some instances, actual prevalences may be higher for all risk factors. For instance, the quoted $34 \%$ prevalence of metabolic syndrome is based upon NHANES 2003-2006 figures, and is now significantly higher in the USA, reaching 53\% in some subpopulations. Hence in the 2011 Update on Heart 
Disease and Stroke Statistics, ${ }^{69}$ the members of the writing group noted that "prevalence and control of traditional risk factors remains an issue ..." The prevalence of obesity may be rising $\approx 1.4 \%$ every 2 years in America, with the current prevalence of visceral obesity at $\approx 53 \%$. $^{70}$

The magnitude of the burden of risk factors may in fact be underestimated. Recently 1,933 middle-aged subjects in the community-based Heart Strategies Concentrating on Risk Evaluation (Heart SCORE) study were evaluated for the seven characteristics - behaviors and factors - of ideal cardiovascular health as defined by the AHA. ${ }^{71}$ Only one participant $(0.1 \%)$ met all of them, and fewer than $10 \%$ of met $\geq$ five components of ideal cardiovascular health. Thirty-nine subjects $(2.0 \%)$ had all four components of ideal health behaviors, while $27(1.4 \%)$ had all three components of ideal health factors (Table 1). About $81 \%$ of their cohort was overweight or obese, possibly due to a high proportion of blacks (44\%), and low prevalence of cardiovascular health was noted in all subgroups: ethnicity, gender, education, and income. Blacks had $82 \%$ lower odds of having $\geq$ five components of ideal cardiovascular health (odds ratio 0.18 , 95\% confidence interval $[\mathrm{CI}]: 0.10$ to $0.34 ; P<0.001$ ). These numbers offer a telling summary, and suggest that when risk factors are carefully evaluated, a truer, even more gruesome index of the present risk burden emerges. Indeed, in this study one limitation was the possibility that the level of cardiovascular risk in the general population might even be higher, because volunteer participants had a relatively low prevalence of tobacco use and a high educational level.

Epidemiologists identify three phenomena now accelerating cardiovascular risk: obesity, diabetes, and the progressive aging of the population. ${ }^{72-76}$ The first two, obesity and diabetes, including metabolic syndrome, respond to primordial, primary, and secondary prevention. The third, a higher proportion of the elderly within the population, is of course, untreatable. Reversal of traditional risk factors in elders is, however, beneficial and presents therapeutic opportunities.

The level of cardiovascular risk in children and young adults is also of major concern, ${ }^{77,78}$ with one-third of this population overweight or obese. As a result of an epidemic of childhood obesity and the rising appearance of diabetes type 2 in the pediatric age group, the number of risk factors now found in children is alarming. Physical activity is below recommended levels in $17 \%$ of adolescents, and $20 \%$ of this population has at least one abnormal lipid value. ${ }^{79}$ Cardiovascular risk, even in the pediatric age group, forms a continuum that has been carefully tracked over the human lifetime, with dominant and close associations between risk factors, CHD risk, and poor outcomes along the way. ${ }^{80,81}$

\section{Screening and control of cardiovascular risk}

Despite the high burden of cardiovascular risk, screening receives a disproportionately small amount of attention and support. Given the comparative death rates and numbers succumbing to cardiovascular disease (CVD), cancer, and $\mathrm{HIV}$, the screening frequencies and filters for asymptomatic CVD (BP, ambulatory monitoring, lipid profiles, advanced lipid testing, others) are much less favorable than for cancer (with less than $10 \%$ of the funding, based upon breast examinations and colorectal screening data) and HIV than for asymptomatic CVD. ${ }^{82}$ In addition, support and enthusiasm for primary prevention, eg, an initial myocardial infarction (MI), is woefully behind that for secondary prevention (recurrent MIs), although the benefits may be greater over an enlarged population base and longer horizon for the former.

In addition, there is ongoing concern that control of risk factors, although progressively improving, still remains far from evidence-based targets. ${ }^{11}$ A review of NHANES data from 2005 to 2008 recently quoted a prevalence of hypertension in American of $\approx 31 \%$, about $70 \%$ receiving treatment and a control rate of $46 \% .{ }^{83}$ An accompanying report, also from the $\mathrm{CDC}$ for the same period, ${ }^{84}$ estimated $33.5 \%$ of adults had elevations in LDL-C, only $48 \%$ received treatment, and $33.2 \%$ were controlled. Overall, well over half of the population with risk factors does not have them under control, and $\leq 10 \%$ with multiple risk factors have them within target ranges.

Poor adherence is itself a major "risk factor" of substantial complexity and underrated significance. ${ }^{85-88}$ About onequarter of all new prescriptions are not filled, ${ }^{89}$ and fewer than half of patients may eventually be taking the drug prescribed..$^{90-92}$ Therefore, when one discounts the patients who are undiagnosed, undertreated, considers adherence shortfalls, and significant residual risk, discussed further below, it becomes evident that current prevention effectively reduces a far smaller portion of total cardiovascular risk than is customarily believed.

\section{Global risk burden is high}

High burdens of cardiovascular risk exist world-wide, as reported by the Institute of Medicine, ${ }^{5}$ World Heart Federation, and in the EuroAspire studies. ${ }^{93}$ Pediatric obesity has also become a global menace. ${ }^{94}$ Similarly, the worldwide prevalence of diabetes is an imposing problem, 
with a staggering 69\% rise in numbers of affected adults in developing nations anticipated by 2030, over three times greater than in developed countries, ${ }^{95}$ which will tax health care systems everywhere. ${ }^{96}$ All told, while effective prevention of diabetes is well-documented, new data creates uncertainty, implementation is fragmented, and some initiatives remain in conceptual suspension globally. ${ }^{97}$

In the 2011 Heart and Stroke Foundation Report on Canadians' Health, risk burden was considered high. The perceptions of self-reported behaviors were compared to the best estimate of actual prevalence of five general risk factors. They concluded that in four categories Canadians were in denial about their health behaviors. ${ }^{59}$ As noted elsewhere, self-reporting of health habits is often inaccurate because positive behaviors are overreported, and negative habits are underreported. In this instance even as reported, a disparity between perception and actual frequencies were identified (Table 3). A similar table based upon NHANES or EuroAspire data would show even greater discordance in the USA and EU. Of concern are the numbers of patients not asked about their use of tobacco or family history for heart disease, and in whom no weight or blood pressures were recorded by practitioners during visits. In an effort to improve cardiovascular risk factors, a web-based e-tool is available as a smart phone application, My Heart\&Stroke Health App ${ }^{\mathrm{TM}}$, is downloadable from www.heartandstroke. $\mathrm{ca} /$ mobileapps, or the interactive evaluation is also offered at www.heartandstroke.ca/risk. The reader will notice the similarity to the American Heart Association's Life's Simple $7^{\mathrm{TM}}$, indicating an endorsement by the HSF of the power and necessity of primordial prevention.

\section{Risk burden in developing nations}

All of the above generally applies to poorer developing nations, which have been included in the epidemic of chronic disease that sweeps across the world. Traditionally noncommunicable disease (NCD) deaths have been overshadowed by those from communicable diseases (CD), and therefore NCDs have not been included in development goals in such countries. ${ }^{98}$ There is no nation in which the prevalence of cardiovascular disease will not rise in the next decade; only in sub-Saharan Africa does the prevalence of CD exceed or equal that of NCD. NCD accounts for about $60 \%$ of all deaths globally, and cardiovascular disease is a major cause. The relative rise in world-wide incidence of CVD is basically due to lower fetal and neonatal mortality, greater use of tobacco, and increased urbanization, with attendant rises in fat and energy consumption, lower physical activity levels, and, according to INTERHEART data, greater psychological stress. ${ }^{99}$ Currently, emphasizing CD prevention rather than NCD only widens disparities in cardiovascular care to the poor, who have a high risk burden that leaves them vulnerable. ${ }^{100}$ Thus far, conventional ideas concerning risk factors and subsequent development of cardiovascular disease appear to apply to developing nations, but it is unclear whether techniques and goals are relevant. Especially with considerable doubt about how best to control risk factors in a familiar and stable environment, whether we are in fact exporting inapplicable intensive "solutions" for different cultures is currently being debated. In other countries, such as China, it is anticipated that the principles successfully employed in Western nations will also be productive.

Franco et $\mathrm{al}^{101}$ also call attention to the global emergency faced by countries of low- and middle-income, given the high burden of cardiovascular risk, the dire need for primordial prevention, and the multiple potential sources of resistance that impede implementation. The NCD Alliance, with The World Heart Federation as a component of The NCD Alliance, is aggressively urging implementation of the WHO 2008-2013 Action Plan for the Global Strategy for the Prevention and Control of NCDs, as well as the Global Strategy on Diet, Physical Activity and Health, which set forth strategies for primordial prevention.

Table 3 Cardiovascular risk factors as reported in the 201 I Heart and Stroke Foundation Report ${ }^{38}$

\begin{tabular}{lllll}
\hline Risk factor & $\begin{array}{l}\text { Self-reported } \\
\text { perception } \\
\text { (\% participation) }\end{array}$ & $\begin{array}{l}\text { Actual } \\
\text { prevalence } \\
\text { (\%, estimated) }\end{array}$ & $\begin{array}{l}\text { Not assessed by } \\
\text { health care provider (\%) }\end{array}$ & $\begin{array}{l}\text { Impact upon } \\
\text { life expectancy } \\
\text { (years) }\end{array}$ \\
\hline Smoking & 23 & 20 & 37 & -13.9 \\
$\begin{array}{l}\text { Obesity } \\
\text { (age 18 or over) }\end{array}$ & 18 & 24 & Weight: $40 \%$ & -4.0 \\
Physically inactive in leisure time & 31 & 48 & 44 & -3.6 \\
High blood pressure & 17 & 19 & 18 & -2.4 \\
Vegetable and fruit intake <5+/day & 39 & 54 & 52 & -1.3 \\
\hline
\end{tabular}

Data from the Heart and Stroke Foundation (HSF) poll of 2000 Canadians performed in December 2010, self-reported from the 2009 Canadian Community Health Survey, or from the 2007-2009 Canadian Health Measures Study. 


\section{Coronary heart disease begins in childhood and progresses throughout life}

Primordial, primary, and secondary prevention are underpinned by convincing evidence that atherosclerosis begins in infancy, ${ }^{102}$ has an incubation period of decades, and progresses throughout an individual's lifetime. At the same time, evidence is also accumulating that risk factors, such as obesity, have prenatal beginnings. ${ }^{69}$

The pathology of early stages of atherosclerosis in children and adolescents is a function of the same traditional risk factors that affect adults. Worsening of risk factors accelerates the disease, but their improvement slows or is even capable of reversing the process. The longer the exposure to, and the greater the intensity of, the risk factors involved, the higher the atherosclerotic burden will become. ${ }^{103,104}$

Epidemiological, pathological, ${ }^{105,106}$ and risk factor data $^{79,107,108}$ confirm that the atherogenic process already incubates during childhood, and can even be detected in utero. ${ }^{109}$ Fatty streaks, a common early manifestation of atherosclerosis and well-developed in many young adults, are present in $37 \%$ of asymptomatic, otherwise healthy organ donors from ages $20-29 .{ }^{110}$ These findings are consistent with reports of significant coronary atherosclerosis in the majority of young soldiers who died on the battlefield. ${ }^{111,112}$

The Bogalusa Heart Study (BHS), directed by Dr Gerald S Berenson for the past 39 years, has clearly established the significance risk factors have in youth, now well described in about 1000 publications and four books. ${ }^{78,80,113-117}$ Observations from the BHS show that the major etiologies of adult heart disease and atherosclerosis: hypertension, dyslipidemia, and obesity, begin in childhood, with anatomic changes evident by $5-8$ years of age. Autopsy studies demonstrate lesions that correlate with risk factors ${ }^{116}$ One of the most striking of the findings in the Bogalusa study, in addition to the early presence or progression of childhood risk factors, was their tracking into adulthood. ${ }^{118} \mathrm{~A}$ recent contribution is a retrospective cohort study of adults 19-39 years of age who were followed for an average of 17 years since childhood. ${ }^{115}$ Adverse levels of glucose homeostasis variables in childhood persisted into adulthood but also predicted adult pre-diabetes and diabetes, and correlated with cardiometabolic risk factors. In this regard, a significant relationship between excess visceral fat, dyslipidemia, hypertension, and elevations in CRP levels has also been established in children and adolescents. ${ }^{119-121}$

Magnussen et a ${ }^{122}$ studied changes in adiposity (BMI, waist circumference, skinfold thickness), fitness (bicycle testing), plasma lipids (TC, LDL-C, HDL-C, TG), smoking and socioeconomic status (parental education level) in 539 young Australians in the Childhood Determinants of Adult Health Study. Baseline measurements were made in 1985 when participants were 9, 12, and 15 years old, and again between 2004 and 2006. Among those with hypertriglyceridemia in youth, $79 \%$ of males and $97 \%$ of females had normal values $\sim 20$ years later. The majority of those with elevated levels of HDL-C at follow-up had normal levels at baseline. Both TC and LDL-C tended to be more constant, and most youngsters with elevations at baseline had them at follow-up, later in life. When participants had adverse lipid profiles at baseline, if they also gained weight, or continued to smoke during the interval, at follow-up they were more likely to have dyslipidemia as well. Similarly, those without adverse lipid profiles at baseline were significantly more likely to have dyslipidemia later if they gained weight or continued to smoke in the interim. Last, those who had normal lipid profiles at baseline, but who developed higher risk at follow-up had greater gains in weight, reduced fitness, and failed to rise socioeconomically. Also of note was the association of long-term aerobic exercise training and upward social mobility from youth to adulthood, with higher HDL-C levels. The data suggested that, whether dyslipidemia was present or not in youth, risk factor modification significantly impacted risk when those individuals became adults some 20 years later.

The Pathobiological Determinants of Atherosclerosis in Youth (PDAY) studied 2,876 persons 15-34 years of age who died of external causes, and found a strong concordance between coronary and aortic atherosclerosis and risk factors. ${ }^{123-126}$ The early PDAY score of modifiable risk factors and its variation predict risk in youth and may be useful in identifying high risk individuals.

Recent imaging studies reflect the same pathophysiology. In the Coronary Artery Risk Development in Young Adults (CARDIA) study ${ }^{81}$ of 3,258 individuals ages $18-30$, the $87 \%$ with nonoptimal lipids were about 5.5 -fold more likely to have coronary artery calcium deposits 20 years later, compared with participants with the lowest lipid values. Coronary calcium prevalence was $8 \%$ in young adults with optimal LDL-C levels ( $<70 \mathrm{mg} / \mathrm{dL}$, or $<1.81 \mathrm{mmol} / \mathrm{L}$ ), but $44 \%$ in those with LDL-C cholesterol levels of $\geq 4.14 \mathrm{mmol} / \mathrm{L}$ ( $\geq 160 \mathrm{mg} / \mathrm{dL}$ ). HDL cholesterol was also predictive. Hence, the CARDIA trial demonstrated that nonoptimal levels of LDL-C and HDL-C during young adulthood are independently associated with CHD 2 decades later.

The Cardiovascular Risk in Young Finns study ${ }^{127}$ sought to determine whether childhood risk factors were 
associated with a 6-year change in carotid intima-media thickness (CIMT) in young adulthood independent of the current risk factors. In 1,809 subjects who were followed for 27 years from baseline (in 1980, age 3-18 years), CIMT was measured both in 2001 and 2007. Childhood risk factors assessed included LDL-C, HDL-C, BP, obesity, diabetes, smoking, physical activity, and frequency of fruit consumption. In participants with zero, one, two, and $\geq$ three risk factors, CIMT increased during 6 years by $35,46,49$, and $61 \mu \mathrm{m}(P=0.0001)$. This relationship remained significant after adjustment for adulthood risk. Of the individual childhood variables, physical inactivity and infrequent fruit consumption were associated with accelerated CIMT progression after adjusting for the adult risk factors. The associations of childhood lipid values and BMI with CIMT progression became nonsignificant when adjusted for current (adulthood) risk factor levels. A composite childhood risk factor score was also associated with CIMT progression and this score remained significant in adulthood after adjustment. In those risk factors with greater relative importance of adult values - HDL/LDL ratio and obesity - correction of adverse childhood factors in adulthood appeared to attenuate the ill effects of childhood burdens. The data therefore suggest that interventions to improve lipid and weight abnormalities between youth and adulthood would be productive.

Data from The Cardiovascular Risk in Young Finns Study, the Childhood Determinants of Adult Health Study, the Bogalusa Heart Study, and the Muscatine Study for the International Childhood Cardiovascular Cohort (I3C) Consortium were combined in order to investigate the age at which risk factors influenced CIMT later in adulthood. ${ }^{128}$ All together, 4,380 participants had TC, BP, BMI, TG measured from age 3-18 years, and CIMT was performed in adulthood, ages 20-45 years, mean follow-up 22.4 years. The number of childhood risk factors was predictive of higher CIMT when measured at ages 9, 12, 15, and 18 years, whereas associations with risk factors measured at 3 and 6 years were not significant. The greater the number of risk factors, the higher was the probability of a raised CIMT. A 9-year-old obese child with two additional risk factors would have double the risk of a CIMT in the top decile of the adult distribution, as compared with a child without cardiovascular risk factors. ${ }^{129}$ The accompanying editorial noted that i) these data underscore the need to begin population-wide risk reduction in early youth, which is applicable to whites and blacks world-wide; ii) screening is meaningful from age 9 years onward; iii) current data indicate the absence of risk factors in children is associated with a low likelihood of atherosclerosis; iv) screening for dyslipidemia, BP, and BMI in children is supported; but v) how to select those children for aggressive risk reduction remains unknown. Details about treatment of pediatric dyslipidemia are discussed in recent reviews. ${ }^{130-137}$

These studies are consistent with a model in which atherosclerotic lesions, initially in the form of fatty streaks, advance seamlessly over decades to more mature, fibrous plaques found later in life. The disease progresses, at different locations with variable rates, through adulthood ${ }^{110-114,123,138,139}$ Intracoronary ultrasound studies in donor hearts demonstrates coronary artery plaques $\geq 0.5 \mathrm{~mm}$ in $17 \%$ of hearts in 13-19 year olds, which rises to $60 \%$ in $30-39$ year olds. ${ }^{110}$ The speed of progression depends not only upon the time of appearance of the risk factor, but also strongly depends upon the number of risk factors present. By the time lesions are identified, or symptoms appear, the disease is diffuse, elevations in systemic biomarkers may be present, and pathology has been in progress for years. Although a significant portion of the total duration of the disease precedes the diagnosis, the accelerated rise in risk - and events - may be skewed toward later years. This long asymptomatic period, traditionally ignored, needs greater attention and simultaneously provides untapped opportunity.

Since lifestyles and behaviors affecting cardiovascular risk are learned early in life, health education should be emphasized at that time. ${ }^{78}$ Epidemiologists, preventive and pediatric cardiologists, and pathologists all agree that up to $90 \%$ of heart disease can effectively be prevented, and promotion of healthy lifestyles, nutrition and adequate exercise should begin in childhood. Moreover, while risk factors are already significant in young adults (59\%) and LDL-C elevations are not uncommon, screening remains inadequate. ${ }^{140}$ Among 20,266 fifth-grade children from the Coronary Artery Risk Detection in Appalachian Communities (CARDIAC) Project (796), 71.4\% of children met the National Cholesterol Education Program (NCEP) guidelines for cholesterol screening on the basis of positive family history. Of those, $8.3 \%$ had an LDL-C $\geq 130 \mathrm{mg} / \mathrm{dL} ; 1.2 \%$ of them were eligible for treatment with an LDL-C $\geq 160 \mathrm{mg} / \mathrm{dL}$. Of the $28.6 \%$ who did not have a positive family history, ie, did not meet NCEP guidelines, $9.5 \%$ had an LDL-C $\geq 130 \mathrm{mg} / \mathrm{dL}$, and $1.7 \%$ with LDL-C $\geq 160 \mathrm{mg} / \mathrm{dL}$ warranted treatment. Therefore, many children with dyslipidemia are being overlooked, since family history is not an effective indicator for screening. Weight was not a variable in the study. 
While the composition of the "ideal diet" for cardiovascular protection continues to be debated, ${ }^{63}$ it is not the uncertainty of dietary efficacy upon lipid levels to lower risk, nor lack of knowledge about other risk factors upon cardiovascular outcomes, but rather ineffective implementation that impedes meaningful progress. Unfortunately, individual-, school-, and community-based programs thus far have had mixed results for a number of reasons. ${ }^{125,141}$ The rise in pediatric obesity is of concern to pediatricians. ${ }^{142-144}$ and endocrinologists. ${ }^{145}$ Despite the evidence linking early presence of risk factors and adult risk, confirmatory data from other studies, and recent emphasis on primordial prevention, relative inaction on the part of public health authorities continues. ${ }^{117}$ Dr Berenson, with a half-century of experience, aptly summarizes the present status of preventive cardiology as a "hard sell", despite overwhelming acceptance and vocal support.

\section{Global cardiovascular risk scoring for stratification}

For primary prevention patients, guidelines recommend, and many physicians use, the classic Framingham Risk Score (FRS) to objectively estimate the absolute risk of a coronary event (CHD) during a period of 10 years $^{146-149}$ Inputs of age, gender, total cholesterol, tobacco use, HDL-C, $\mathrm{TC}$, systolic blood pressure, and treatment status are entered into multivariable equations. The combination of risk factors generates a risk estimate for fatal and non-fatal CHD events, and subjects are classified into high-risk ( $\geq 20 \%$ risk), intermediate-risk (10\%-20\%), and low-risk $(<10 \%)$ categories. A number of global risk score alternatives to FRS exist based upon different cohorts - SCORE, ${ }^{150}$ QRISK ${ }^{151}$ ASSIGN, ${ }^{152}$ the 2001 ATP-III Risk Estimator (FRS-based) ${ }^{153,154}$ and PROCAM ${ }^{155}$ - as well as the Reynolds Risk Score incorporating CRP for women ${ }^{156}$ and for men ${ }^{157}$ (Table 4). Risk scoring is a considerable improvement over personal physician judgment, and has been used for stratification and to guide evidence-based treatment. ${ }^{158-161}$ Each scoring system is most accurate when applied in the population used for its development. Calibration - the extent of agreement between what is observed and predicted - depends upon prevalence of risk factors and coronary event rates in given populations. For American Indians and Americans of Asian-, Chinese- and Hispanic-descent, the FRS overestimates risk, which may be corrected with recalibration. FRS scores are clinically validated, ${ }^{154}$ globally accepted, and have withstood the test of time. Dent ${ }^{162}$ suggested that the prevalence of CHD in Framingham has been reduced by improvement in risk factors, particularly smoking, and this change in population characteristics accounts for inaccuracies when using FRS tools. He also believes that since the original cohort was Caucasian and socioeconomically advantaged, the FRS may be limited when applied to ethnically diverse groups. ${ }^{162}$ In a variety of populations in North America, average FRS 10-year predictability of CHD is approximately $65 \%-70 \%{ }^{154}$ The FRS does not include a family history of premature cardiac events, an independent and powerful risk factor for CHD. ${ }^{163-168}$ To enhance features and utility, another version of the FRS was developed to predict 10-year global cardiovascular risk and specific events of CVD: coronary, cerebrovascular, and peripheral arterial disease and heart failure. ${ }^{169}$ Unfortunately, there is suboptimal use of scoring systems providing estimates of absolute 10-year risk, with only $17 \%-47 \%$ of practitioners performing such assessments. ${ }^{170-173}$ An informal survey suggests actual use among cardiologists and internists may even be lower. ${ }^{174}$ A recent preliminary report from the PARADIGM registry indicated that as many as $65.7 \%$ of high-risk patients in Canada were being misclassified, with about $34 \%$ of primarycare physicians using the FRS, $28.5 \%$ relying upon clinical judgment, while others are misapplying CRP recommendations based upon JUPITER findings. ${ }^{175}$

While the FRS represents a remarkable advance in primary prevention, is well-known, well-studied, and is in widespread use, some shortcomings have been identified, and there are no randomized controlled trials showing that its use improves outcomes. ${ }^{162,176,177}$ In some European regions and in low-risk populations the FRS may overestimate risk by a factor of 2.87 , but underestimate it by a factor of 0.43 in high-risk populations. ${ }^{178} \mathrm{In}$ women who sustained their first myocardial infarction, 95\% had FRS scores in the low risk category, with the remaining $5 \%$ in the intermediate category. ${ }^{179}$ Using the NCEP-ATP III/Framingham risk estimator, ${ }^{153} 17 \%$ of women in this cohort were ineligible for statins. According to the Framingham Heart Study, 39\% of asymptomatic women over age 50 will eventually suffer a cardiovascular event, but the Third National Health and Nutrition Examination Survey ${ }^{180}$ indicates that just $0.9 \%$ of asymptomatic women will be in the high-risk FRS category. ${ }^{27}$ Using the ATP III tool, for most combinations of risk factors, the majority of nonsmoking men $<45$ years of age and nearly all women $<65$ years of age will have 10 -year risks $<10 \%$, leaving many young individuals with sizeable risk burdens that remain untreated. ${ }^{181}$ FRS may predict satisfactorily in $33 \%$ of women, and in $85 \%$ of men, for a combined prediction rate of $63 \% .{ }^{182}$ The lowest quoted is about $11 \% .{ }^{183}$ The c-statistic, a number which reflects how a model discriminates 


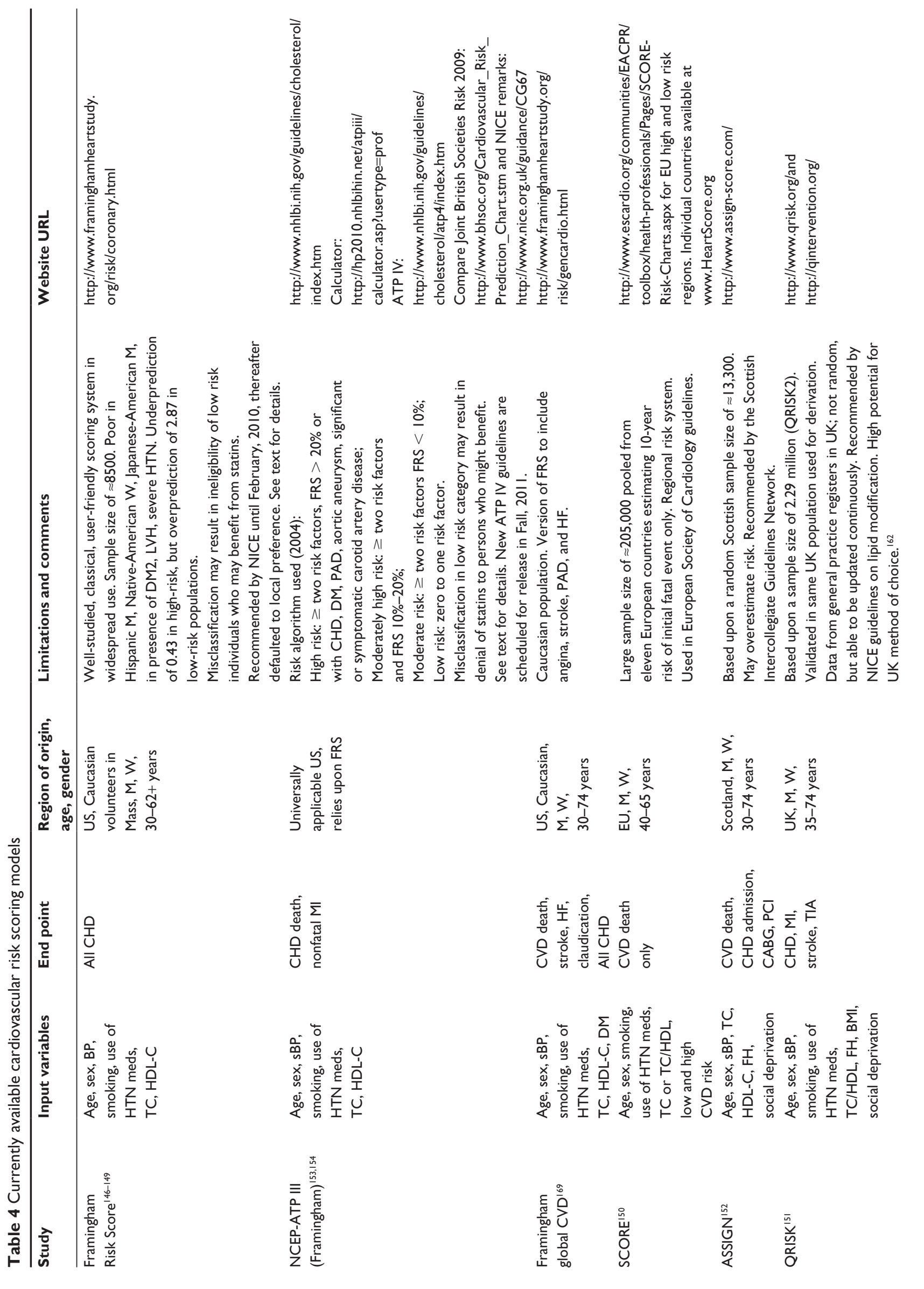



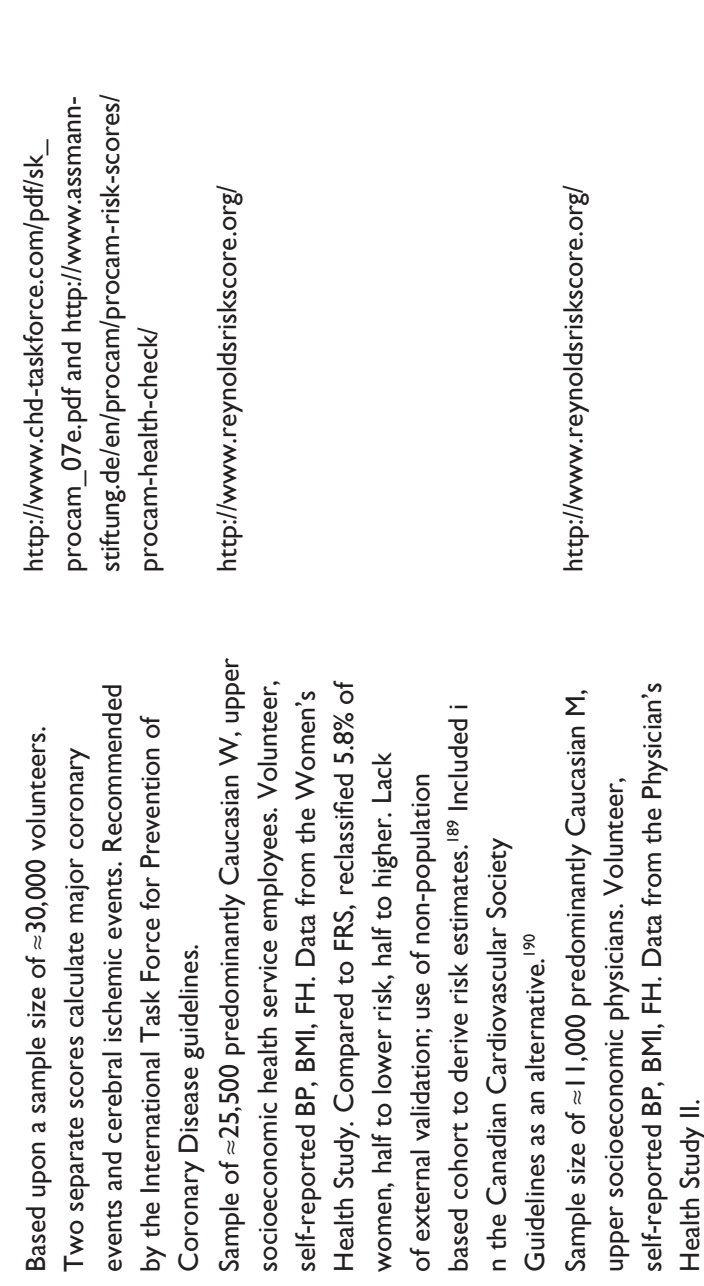

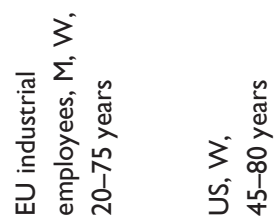

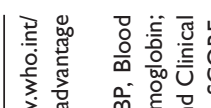

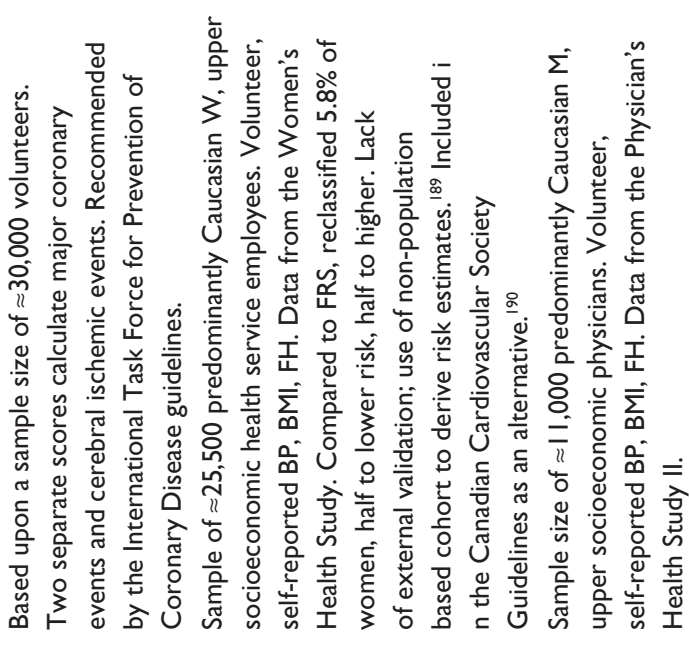

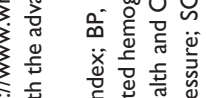

势

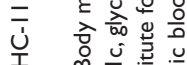

它语

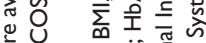

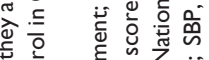

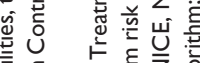

可

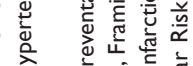

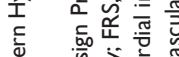

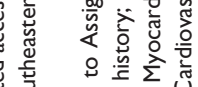

仓 Z U

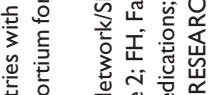

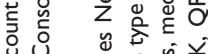

它品

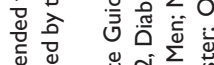

总范

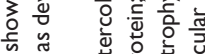

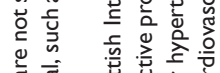

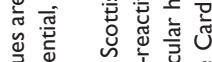

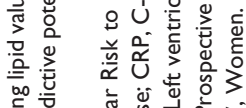

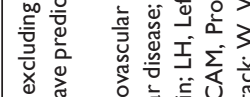

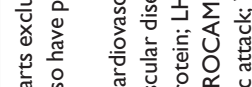

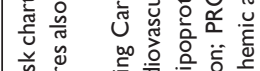

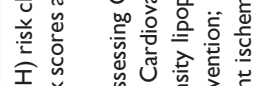

¿ ठ

ปร

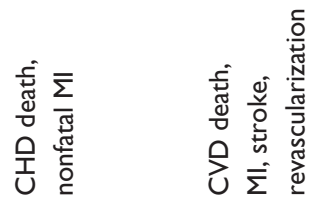

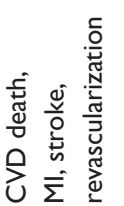

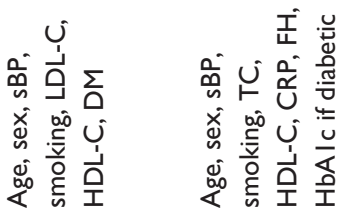

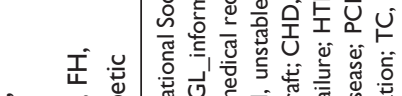

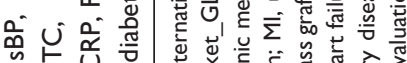

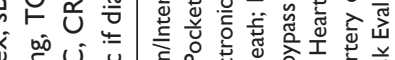

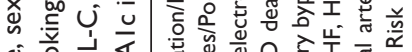

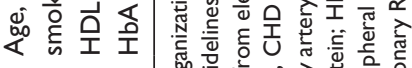
Oั

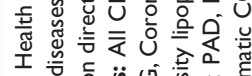

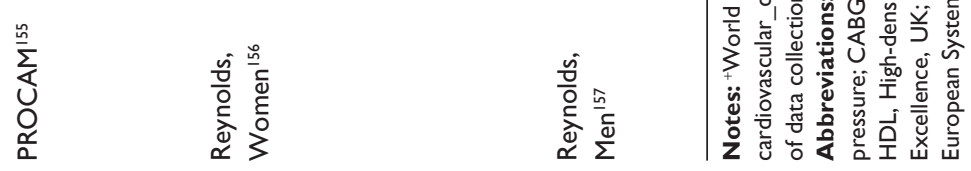


risk for future events, is $0.7-0.8$ for the FRS, ${ }^{184}$ moderately good - a random coin flip is 0.5 . Since CHD develops over decades, a 10-year horizon is insufficient to identify a significant group of people with relatively low risk at the time of scoring, ${ }^{27,185}$ particularly young men. ${ }^{181,186}$ In one study, about $45 \%$ of enrollees between 32 and 47 years of age had a low 10-year predicted risk, but a predicted lifetime risk of $\geq 39 \% .{ }^{187}$ Guidelines for primary prevention in women. ${ }^{188}$ and the NECP Adult Treatment Panel III ${ }^{153}$ have endorsed the use of lifetime risk in younger adults.

According to data using coronary $\mathrm{CT}$ angiography, about two-thirds of women and one-third of men with substantial coronary disease may be missed when the FRS misclassifies them as low risk, ${ }^{192-194}$ because the initial physician is dissuaded from further screening. ${ }^{194}$ Over $75 \%$ of CHD events in men and over $90 \%$ of CHD events may be misclassified in women. In addition, more than $25 \%$ of patients who are being treated with statins may have no detectable plaque on such imaging.

The 2010 ACCF/AHA Guidelines for Assessment of Cardiovascular Risk in Asymptomatic Adults ${ }^{32}$ assigned a Class I, LOE B recommendation for global risk scoring, advising that all asymptomatic adults without a clinical history of CHD should have their risk assessed using this method.

Recent discussions about screening for asymptomatic CHD and merits of scoring systems have raised unresolved issues beyond the scope of this review. For instance, how valid are claims of improved prediction beyond the FRS? One analysis showed that many such studies had limitations in design, analysis, or reporting that made improved predictive ability doubtful. ${ }^{195}$ It should be noted that the Reynolds Risk Score, ${ }^{156}$ which uses CRP levels and family history, and for which required metrics of adequate discrimination, calibration, internal validation, and reclassification have been satisfied, was not one of these. Another question is whether use of a risk-estimation system helps in risk factor reduction, as compared with graded treatment of each risk factor to guideline thresholds. ${ }^{196-197}$ Should asymptomatic patients be screened for atherosclerosis at all, since there is little evidence clinical outcomes improve by doing so, but instead simply administer a polypill to adults of a certain age or dispense free statins in fast-food restaurants? One recent commentary concluded it is worthwhile to screen. ${ }^{198}$ The accompanying editorial ${ }^{199}$ observed that cardiovascular screening tests should be proven to prevent clinical disease, and that ability to predict or reclassify risk is secondary. One valid technique, they continued, is using the screen to identify subjects thought to be at high risk, and randomize them to treatment or control, ${ }^{200}$ as was done in the JUPITER trial. ${ }^{199}$ Papers that appeared before JUPITER was published, and the subsequent analyses that followed, mentioned insufficient improvement in reclassification when CRP was added to scoring systems. ${ }^{201,202}$ Results of large randomized clinical trials, such as JUPITER, provide strong, direct evidence of efficacy. Several recent discussions concerning merits of nontraditional risk factors are based upon statistical parameters. ${ }^{203}$ In the clinic, practical reasons to use biomarkers are a) to refine a clinical decision that leads to an evidence-based change in therapy leading to improved quality of life, morbidity, or mortility, and/or b) to motivate a patient to understand, partner, and make lifestyle changes or adhere to drug or device therapies.

Use of multiple biomarkers to refine prediction of cardiovascular events has met with variable success. ${ }^{204-209}$ Outcomes depend upon the markers selected, and is also a function of the population studied, particularly age, and the stage of the disease process. Recently multivariable regression was used in two middle-aged European populations totalling 10,466 participants, adding a 30-biomarker score which included $\mathrm{N}$-terminal pro-brain natriuretic peptide, C-reactive protein, and sensitive troponin I to a conventional risk model. ${ }^{208} \mathrm{~A}$ rise in 10-year risk estimation for cardiovascular events and significant improvement in reclassification was reported.

\section{0-year risk vs lifetime risk}

For the reasons discussed above, when risk scores are used to guide treatment, a considerable number of asymptomatic, low-risk individuals who may benefit from statin drug therapy over a period of time may not qualify. The current evaluation algorithm consists of dividing individuals without clinical heart disease into low-, intermediate-, and high-risk categories. Those with 10 -year risks over $20 \%$ are begun on statin therapy after evaluation; intermediate risk individuals $-10 \%$ to $20 \%$ - are given drugs for risk reduction and/or further noninvasive testing continues for additional stratification; low-risk persons are generally advised about diet and lifestyle modification. Within this paradigm of targeting higher-risk individuals, approaches to improve the yield have included a) lowering the target LDL-C level; b) lowering thresholds for treatment in each risk group; c) greater use of imaging to identify those individuals misclassified by absolute risk scoring; and d) replacing the 10-year risk horizon with lifetime risk.

The strengths of the relationships between individual risk factors with death vary not only with the risk factor, 
but with the time frame and gender. ${ }^{210}$ Lloyd-Jones and collaborators have studied the use of lifetime risk for CHD extensively. ${ }^{27,52,165,168,185,210,211}$ These investigators noted that individuals younger than age 40-50 in a low-risk category with an initial FRS $<10 \%$ may have a surprisingly large risk burden and a high lifetime risk for CHD which may not be appreciated using current stratification techniques. ${ }^{27,211}$ For example, the Coronary Artery Risk Development in Young Adults Study found that $>90 \%$ of individuals 32-47 years of age had a predicted FRS risk of $<10 \%$, yet had a lifetime risk of $\geq 39 \%$. ${ }^{187}$ According to NHANES data from 2003-2006, about $56 \%$ of American adults have a low 10-year risk but a high lifetime risk, only $18 \%$ have high short-term predicted risk, and $26 \%$ have both low short-term and low lifetime predicted risk. ${ }^{213}$ Moreover, just $8 \%$ of men and $14 \%$ of women enjoy optimal risk factors associated with a low lifetime risk for heart disease. ${ }^{213}$ Some of the errors in risk estimation by the FRS arise from unpredictable changes that occur in risk factors over time, such as blood pressure and insulin resistance, which may increase relatively quickly with age. ${ }^{214}$ Others arise from changes in the competing risk of death from causes other than CHD, such as tobacco use. Lifetime risks for CVD are comparable to those from extremely lethal diseases, and are generally higher than risks related to many cancers. At 50 years of age, however, the absence of traditional risk factors is associated with extremely low lifetime risk and significantly greater longevity. ${ }^{27,168}$ People with favorable risk factors enjoyed much lower lifetime risks for CVD death than those with unfavorable profiles (zero vs $\geq$ three risk factors, $20.5 \%$ vs $35.2 \%$ for men; $6.7 \%$ vs $31.9 \%$ for women), along with longer survival (men, $>35$ vs 26 years; women, $>35$ vs 28 years). ${ }^{168}$

A striking example of the limitations of current risk assessment in relation to actual lifetime risk was provided by the work of Berry et al. ${ }^{187}$ They stratified 2,988 persons $\leq 50$ years of age from the Coronary Artery Risk Development in Young Adults (CARDIA) study ${ }^{181,215-217}$ and 1,076 similar individuals from the Multi-Ethnic Study of Atherosclerosis $(\mathrm{MESA})^{217-219}$ into three groups: low 10-year $(<10 \%)$ risk/ low lifetime $(<39 \%)$ risk; low 10-year/high lifetime risk ( $\geq 39 \%$ ); and high 10 -year $(\geq 10 \%)$ risk or diagnosed diabetes mellitus (DM2). Among those with a low FRS, there were just as many participants with a high lifetime risk as a low lifetime risk, which implies that as many as half of individuals in this large low-risk group may fail to receive beneficial therapy.

In this investigation, coronary artery calcium (CAC) was also used to assess the degree of subclinical disease at baseline and after the study periods. In CARDIA, a CAC was performed at year 15 and year 20; in MESA, a CAC was performed at baseline, at about 22 months in $50 \%$ of participants, and at about 40 months in the remaining half. In addition, CIMT was performed in year 20 in the CARDIA study, and at baseline in MESA. In participants with a low 10 -year risk, the vast majority in the study, individuals with a low lifetime risk compared with a high lifetime risk had significantly smaller dimensions of common and internal carotid intima-media thickness, as well as a lower prevalence and progression of coronary artery calcium. ${ }^{187}$ The differences in CIMT dimensions in younger adults were believed to ultimately correspond to considerable increases in cardiovascular risk over the years. Hence, exposure of individuals to higher risk factor intensities over a substantial period of time leads to accumulation of atheromatous changes, eventually reaching the threshold that produces clinical events at older ages. ${ }^{187,216}$ However, those individuals without risk factors at age 50 have a lifetime risk of CVD of approximately 5\% over a generously extended lifespan. ${ }^{27}$

Consideration of lifetime risk is clearly important, not simply philosophically, but in treating patients in the real world. In view of these data, reports suggesting that statins are being overprescribed on the basis of 10-year risk estimations, ${ }^{220}$ or even a single CAC study, ${ }^{221}$ are of concern, and at the least, suggest further trials are needed. Not only in America, but globally, the facts indicate otherwise - that statins are underprescribed, and strikingly so, given the level of prevailing risk. ${ }^{222}$ Moreover, if low CAC scores only provide a "warranty" for a period of 4-5 years, ${ }^{223}$ retesting at such intervals to assess need for statins in patients who are likely to undergo additional diagnostic imaging could significantly raise the burden of radiation exposure, particularly if CAC screening widens. ${ }^{224-226}$

Nambi and Ballantyne, ${ }^{227}$ editorializing on the broader significance of these findings in risk stratification, also observed that within classically-measured low-risk cohorts of the "healthy" population, there was a significant difference in observed risk over 14 years of follow-up in their own work, ${ }^{228}$ and concluded that the evaluation of "low-risk" patients needs to be reassessed.

A new multi-level risk stratification proposal has been made using the FRS as the first tier, lifetime risk as the second filter, and CRP levels, with CAC and CMIT in a third tier to guide LDL-C-lowering therapy. ${ }^{227}$ These are the major tools that are considered important for risk assessment Recent data reflecting improved long-term safety and cost-effectiveness 
of statins, even at FRS risks $\approx 10 \%$, are additional reasons favoring statin therapy when predicted risk is in the lower end of the spectrum. ${ }^{76}$

The data mentioned above are consistent with increasing awareness that the prevalence of ideal cardiovascular health in the population is low, and the greater number of cardiovascular events will ultimately rise among individuals who are not in a high-risk category. ${ }^{2}$ The information presented also adds to the growing body of evidence underscoring the importance of not only preventing development of risk factors at the earliest opportunity, but also screening sooner, stratifying more efficiently, and using intensive therapies to reduce risk.

\section{Early, aggressive treatment is the new imperative}

Early detection of atherosclerotic disease and refinement of risk assessment has been accompanied by a recent sea change in the thinking about the timing and aggressiveness of treatment of risk factors, in both ostensibly healthy individuals who are not investigated further than "routine" LDL-C levels, and those with established CHD. 2,27,28,52,185,229-232 The regression line for major statin studies shows that LDL-C reduction produces benefits in a linear fashion approaching "physiological" levels (see section below). The Cholesterol Treatment Trialists study suggested outcome improvement in statin-treated patients with baseline LDL-C $\approx 80 \mathrm{mg} / \mathrm{dL} .{ }^{233}$ The evidence for aggressive LDL-C lowering for high risk patients has been recently discussed elsewhere. ${ }^{234,235}$ Notably, Steinberg and coworkers ${ }^{138}$ called attention to the urgent need for earlier intervention in reducing risk factors, especially LDL-C, adding to the consensus.

Due to the evolution of disease-reactive medical care, escalation of clinical assessment and treatment usually follows the finding of either abnormal biomarkers or symptoms. Waiting until this occurs in CHD, with its long incubation period, is a prescription for certain failure of "primary" care. By the time elevations in LDL-C or CRP occur, especially at levels mandating therapy using current guidelines, the disease is widespread, relatively advanced but still often inaccessible to customary techniques used for detection. Consequently, the improbability of intervening early enough has generated skepticism about whether prevention is ever "primary", ${ }^{236}$ For instance, studies using intravascular ultrasound (IVUS) $)^{237-242}$ and intracoronary optical coherence tomography ${ }^{243,244}$ amply demonstrate plaque in various stages of evolution invisible to routine coronary angiography due to limitations in resolution and view.
Proprotein convertase subtilisin-like/kexin type 9 serine protease $(P C S K 9)$ is a gene encoding PCSK9, a protease expressed in the liver and intestine in adults. The liver releases PCSK9 into the circulation, concentrations of which correlate with BMI, triglyceride, TC, and LDL-C levels, as well as blood pressure, fasting blood glucose, and gamma-glutamyl transferase levels. The protease is a molecular chaperone, bindling to the epidermal growth factor-like domain A of the LDL receptor, redistributing the LDL receptor from the plasma membrane to an endosomal/lysosome pathway for degradation. ${ }^{245}$ PCSK9 thereby regulates LDL receptor levels on the cell surface, particularly in hepatocytes. ${ }^{246}$ Individuals with PCSK9 loss-of-function mutations have LDL-C levels about 28\% lower than nonvariants. ${ }^{247,248}$ About $3 \%$ of the population may have these variations. Such changes correspond to an $88 \%$ fall in risk for CHD events, nearly three times the reduction of a 5-year course of statins that reduces LDL-C comparably. On the other hand, gain-of-function mutations in PCSK9 results in diminished net expression of LDL receptors, producing a clinical picture resembling familial hypercholesterolemia. ${ }^{249,250}$ This experiment of nature suggests that favorable levels of LDL-C begun early, and maintained throughout life, are needed for optimum reduction in CVD events. Using the data from PCSK9 studies, Steinberg ${ }^{251}$ calculated that beginning statin therapy much earlier might double the yield and prevent 240,000 coronary events annually, based upon 2009 CHD mortality data. ${ }^{1}$ By any account, benefits associated with the use of statins and other preventive therapies over a 3.7-5.0-year span of a study would only be a fraction of the potential if applied over the total incubation period of CHD, 40-60 years.

In view of the above, aggressive intervention, when indicated, should be offered at the soonest opportunity to prevent disease progression and future coronary events. ${ }^{107,138,252,253}$ The longer lipid-lowering therapy continues, the greater the reduction in relative risk. ${ }^{233}$ Risk factors in youth are growing concerns among pediatricians. The ACCF/AHA 2009 Performance Measures for Primary Prevention of Cardiovascular Disease in Adults ${ }^{254}$ suggested that lifestyle measures begin at age 18 .

Forrester $^{255}$ emphasizes the difference between proof and inference, the need to begin treatment according to the pathogenesis of atherosclerosis rather than initial clinical manifestation, choose a lower physiologic LDL-C target, and adopt a new practical, but more effective, mindset. In view of the above, using CRP as a guide to earlier treatment from randomized clinical trial data, rather than by estimates of added increments in risk factor prediction using scoring 
systems, is pertinent and timely. ${ }^{52,256}$ Rosuvastatin and atorvastatin in higher doses are the preferred agents for both LDL-C- and CRP-lowering ability. ${ }^{257,258}$

\section{Statins in primary prevention}

Measures used in primary prevention customarily include smoking cessation, diet modification, physical activity, weight management, correction of dyslipidemia (lowering LDL-C globally, targeting subfractions of LDL including Lp(a), lowering LDL-P or increasing the size of LDL particles, raising HDL-C globally, targeting subfractions of HDL, reduction of plasma triglycerides (TG) and other TG-rich atherogenic particles), reducing blood pressure, controlling diabetes and insulin resistance in related syndromes, use of aspirin, and treatment of comorbidities that commonly raise CHD risk, such as chronic kidney disease. The WHO MONICA project found that over half of non-fatal MIs in young people are attributable to smoking, ${ }^{259}$ and risk returns to baseline about 5 years after cessation. ${ }^{260}$ For each cigarette smoked daily, the risk of MI rises by $5.6 \% .{ }^{12}$ For every $38.7 \mathrm{mg} / \mathrm{dL}(1 \mathrm{mmol} / \mathrm{L})$ fall in plasma LDL-C, there is a corresponding $21 \%$ reduction in cardiovascular events. ${ }^{261} \mathrm{~A}$ drop in systolic blood pressure of $10 \mathrm{mmHg}$ may result in a decrease of cardiovascular mortality of $20 \%-40 \%,{ }^{262}$ which may be enhanced by using ambulatory blood pressure monitoring. ${ }^{263} \mathrm{~A}$ reduction in risk factors has contributed to the fall in the incidence of MI since 2000, ${ }^{264}$ corresponding to a decrease in the prevalence of smoking from $23.1 \%$ to $19.7 \%$, and a drop in the prevalence of hypercholesterolemia from $17.0 \%$ to $16.3 \%$. A $1 \%$ change in absolute risk may affect $\approx 2.2$ million adults. ${ }^{265}$

\section{Effect of statins on plaque volume}

The use of intravascular ultrasound (IVUS), with a greater sensitivity than coronary angiography, provides a direct and revealing method of assessing the effect of statin drugs upon coronary atheroma. ${ }^{237-241,266,267}$ In recent trials, there was a strong linear relationship between LDL-C values and atheroma progression rate, with a critical reversal level of LDL-C at $70 \mathrm{mg} / \mathrm{dL}$. While the effects of statins upon lesions are evident, the extent of atheroma regression varies (Table 5). In the ASTEROID study, ${ }^{230,242,267}$ rosuvastatin $40 \mathrm{mg}$ lowered LDL-C by $53 \%$ and raised HDL-C by about $15 \%$, accompanied by a significant reduction in atheroma volume in both coronary arteries that were angiographically normal as well as those with visible obstructions. Extension of these data with the use of quantitative coronary angiography (QCA) suggested slowing of progression as well as regression of lesions in different segments of the coronary tree. ${ }^{240}$ Improvement in luminal dimensions, as measured with QCA, correlated with risk of coronary events and mortality. ${ }^{268,269}$ Changes in atheroma volume, stratified above or below a median change of $-21.4 \%$ in CRP levels and $-37.1 \%$ in LDL-C, was greatest in those with reductions in both LDL-C and CRP $\left(-2 \mathrm{~mm}^{3}\right)$, less when CRP fell but not LDL-C $\left(-1 \mathrm{~mm}^{3}\right)$, progressed when LDL-C fell but CRP was high $\left(+2 \mathrm{~mm}^{3}\right)$, and progressed the most when both LDL-C and CRP were high $\left(+8 \mathrm{~mm}^{3}\right) .{ }^{230}$

Serial computed tomography angiography (CTA) was performed in 32 older patients, 24 of whom took fluvastatin after the baseline CTA, with eight subjects who declined statin therapy serving as controls. After a median period of 12 months, plaque volumes were calculated in a $10 \mathrm{~mm}$ segment selected for comparison. Plaque volumes were significantly reduced in the statin-treated group. ${ }^{276}$

These data support a model in which atherosclerosis advances unpredictably in multiple sites at different rates. When LDL-C is lowered with statins, particularly rosuvastatin, the progression of the lesions may wane, some

Table 5 Recent statin regression trials using coronary IVUS

\begin{tabular}{|c|c|c|c|c|c|}
\hline Investigators & Type & Statin used & Exam interval & Primary endpoint & Results (mean \pm SD) \\
\hline \multirow[t]{2}{*}{ Petronio et $\mathrm{al}^{270}$} & $\mathrm{RCT}$ & Simvastatin & 12 months & Plaque volume & $-2.5 \pm 3.0 \mathrm{~mm}^{3} / \mathrm{mm}$ \\
\hline & & Control & & & $1.0 \pm 3.0 \mathrm{~mm} / 3 / \mathrm{mm}$ \\
\hline \multirow[t]{2}{*}{ Tani et $\mathrm{al}^{271}$} & $\mathrm{RCT}$ & Pravastatin & 6 months & $\%$ change in plaque volume & $-14.4 \pm 23 \%$ \\
\hline & & Control & & & $\mathrm{I} . \mathrm{I} \pm 4.6 \%$ \\
\hline ASTEROID 230,242 & Not RCT & Rosuvastatin & 24 months & Change in PAV & $-0.98 \pm 3.15 \%$ \\
\hline \multirow[t]{2}{*}{ Takashima et $\mathrm{al}^{272}$} & Not RCT & Pitavastatin & 6 months & $\%$ change in plaque volume & $-10.6 \pm 9.4 \%$ \\
\hline & & Control & & & $8.1 \pm 14.0 \%$ \\
\hline $\operatorname{cosMOS}^{273}$ & Not RCT & Rosuvastatin & 18 months & Change in PAV & $-5.1 \pm 14.1 \%$ \\
\hline \multirow[t]{2}{*}{ JAPAN-ACS ${ }^{274}$} & $\mathrm{RCT}$ & Atorvastatin & $8-12$ months & $\%$ change in plaque volume & $-18.1 \pm 14.2 \%$ \\
\hline & & Pitavastatin & & & $-16.9 \pm 13.9 \%$ \\
\hline \multirow[t]{2}{*}{ Hirayama et $\mathrm{al}^{275}$} & Not RCT & Atorvastatin & 28 weeks & $\%$ change in plaque volume & $-9.4 \pm 10.3 \%$ \\
\hline & & & 80 weeks & & $-18.9 \pm 14.1 \%$ \\
\hline
\end{tabular}

Abbreviations: RCT, randomized controlled trial; PAV, percent atheroma volume. 
with complete regression. Whether these events are evident is also a function of the sensitivity of the technique used for assessment. ${ }^{277}$ Even when LDL-C is lowered drastically, approaching "physiological” levels, however, some lesions may still progress in many patients. ${ }^{278}$ This would include primary prevention patients, who may be so classified as a result of relatively insensitive techniques, but in whom the process is already under way. While unproven, preliminary evidence suggests that when CRP levels are elevated, especially in patients with metabolic syndrome, residual risk is likely to be high, a correlation may exist with CIMT, and the response to rosuvastatin may be greater. ${ }^{279,280}$

\section{Statins recommended in guidelines}

Several primary prevention guidelines from the USA, UK, and EU are available to assist clinicians, and the use of statins is typically recommended. ${ }^{12,153,158-160,188,281-285}$ According to the last NCEP-ATP III recommendations for primary prevention, statins should be begun when LDL-C $\geq 190 \mathrm{mg} / \mathrm{dL}$ in individuals with low risk, is discretionary when LDL-C is $160-189 \mathrm{mg} / \mathrm{dL}$, and is not advised for ostensibly healthy persons with LDL-C $<160$ unless there are $\geq$ two risk factors. ${ }^{286}$ All are fairly high numbers, considering that $50 \mathrm{mg} / \mathrm{dL}$ might be "physiologic", and soon NCEP-ATP IV will announce modified targets. The ACCF/AHA/ACP 2009 Competence and Training Statement on primary prevention ${ }^{287}$ succinctly reviews the essential role of lipid management, and advises that intensity of therapy match the individual risk for CAD in each patient. The overriding fact of life is that since the vast majority of people will not follow required lifestyle changes, patients with elevated cardiovascular risk will be treated with statins. Statin drugs have revolutionized the practice of cardiology over the last two decades, and there are no equals.

\section{Analysis of statin use in primary prevention}

There are seven systematic reviews examining the use of statins in primary prevention. ${ }^{288-294}$ Mills et $\mathrm{al}^{290}$ pooled results from 19 randomized trials to evaluate the effectiveness of statins in primary prevention. For 19 trials examining all-cause mortality, the relative risk (RR) was 0.93 , and for 17 trials, using major cardiovascular events, the RR was 0.85 . Overall, they concluded statins have a clear role in primary prevention of major events and mortality. Brugts and coworkers $^{291}$ included ten trials involving 70,388 people, $34 \%$ women and $23 \%$ with diagnosed diabetes, all with risk factors but without established cardiovascular disease. Statin therapy lowered all-cause mortality (odds ratio [OR]: 0.88), major coronary events (OR: 0.70), and major cerebrovascular events (OR: 0.81). Mean follow-up time was 4.1 years. Since many trials did not consider death after a morbid cardiovascular event, the $12 \%$ risk reduction in mortality may have been an underestimate. Even though no clinical heart disease was identified, the pooled risk in the study population was high, and overall annual mortality was about $1.4 \%$. Yet the relative risk reduction from statin use for primary prevention was comparable to that for secondary prevention.

A recent meta-analysis of the use of statins and all-cause mortality in high-risk primary prevention was performed by Ray et al. ${ }^{292}$ Eleven randomized controlled trials involving 65,229 participants treated with statins for an average of 3.7 years were found to have no statistically significant improvement in all-cause mortality. In those treated for primary prevention, average LDL-C levels fell from 139 to $98 \mathrm{mg} / \mathrm{dL}$ (multiply by 0.0259 to convert to $\mathrm{mmol} / \mathrm{L}$ ). Compared to a mean placebo death rate of 11.4 per 1000 patient-years, there were only seven fewer deaths for every 10,000 patient-years of statin treatment.

The investigators emphasized the need to tease out patients with preexisting heart disease, because those patients would be the ones known to benefit from statin therapy. Although they were critical of other studies because of the short periods of study, the 3.7 year period of their meta-analysis was also relatively small. While the authors acknowledged statin efficacy in patients with diagnosed CHD, ie, for secondary prevention, they challenged statin use in low-risk patients when applying current prevention guidelines. ${ }^{31,293,294}$ Because this study was a meta-analysis, there was considerable heterogeneity in patient risk and statin use, and some of the data used were old. In addition, the limitations of meta-analyses are well-known, but apply equally to the analyses that found statins do improve mortality in primary prevention..$^{290,291}$

The reaction to this study was muted, with the consensus believing that in a primary prevention cohort, the period of statin treatment involved was insufficient to show more, and that a reduction in CHD mortality, combined with the long incubation period of atherosclerosis evidenced by results of longitudinal studies, justified treatment with statins. Waiting years for treatment in primary prevention would be an imprudent delay, according to most observers.

Bukkapatnam et $\mathrm{al}^{295}$ focused their meta-analysis on women and included 6 studies, including JUPITER, totaling 21,963 moderately hyperlipidemic women given statins for primary prevention. These investigators found that 
the summary risk ratio for any CHD event was 0.78 (95\% CI: $0.64-0.96 ; P=0.02$ ), but there was no demonstrable reduction in all-cause mortality or CHD deaths. They attributed the better results reported in JUPITER, in part, to the stronger predictive power of CRP in women than in men. However, Bukkapatnam, ${ }^{295}$ like Brugts and coworkers, ${ }^{291}$ included data from the Primary Prevention of Cardiovascular Disease With Pravastatin in Japan (MEGA) study, conducted at 924 sites from 1994-2004. ${ }^{296,297}$ This trial randomized 7,832 healthy men and women to pravastatin $10-20 \mathrm{mg}$ with dietary modifications, or dietary modifications alone with a median follow-up of 5.3 years. MEGA found that statin therapy lowered CHD events by $33 \%$, and relative risk for CVD events by $30 \%$. Since the mean fall in LDL-C levels was just $11 \%$, the statin produced an effect greater than would be expected from the reduction in LDL-C. While agreeing with the JUPITER trial discussed below, in that a statin was effective in lowering cardiovascular events in low-risk individuals without heart disease, some caveats are in order. At the time of the study, the prevalence of CHD in Japan was much less than in the countries in which JUPITER was conducted, and pravastatin in the doses used was considerably less potent than rosuvastatin $20 \mathrm{mg}$.

Taylor and coworkers ${ }^{298}$ analyzed randomized controlled trials of statins in primary prevention with a minimum duration of 1 year, a follow-up of at least 6 months in adults, and also excluded $\leq 10 \%$ of participants with a history of CVD. Of the 14 trials included, 11 recruited patients with hyperlipidemia, diabetes, hypertension and microalbuminuria. Statins were associated with a $17 \%$ reduction in all-cause mortality (RR: $0.83,95 \% \mathrm{CI}: 0.73$ to 0.95 ), lowering of combined fatal and non-fatal CVD endpoints (RR: 0.70, 95\% CI: 0.61 to 0.79 ), with variation that was attributed to different populations, statins employed, and reporting (Table 6). The investigators concluded that no significant harm or change in patient quality of life was caused by statins, and they reported sizeable improvement in all-cause mortality, $\mathrm{CHD}$, and stroke events, as well as number of revascularizations. According to their review, there was selective reporting of outcomes, failure to report adverse events, and inclusion of participants with diagnosed CVD. Despite the evident

Table 6 Outcome improvements with statin therapy in primary prevention found in the Cochrane review ${ }^{298}$

\begin{tabular}{ll}
\hline Outcome & Risk ratio $(95 \% \mathrm{Cl})$ \\
\hline Total mortality & $0.83(0.73-0.95)$ \\
Fatal and nonfatal CHD events & $0.72(0.65-0.79)$ \\
Fatal and nonfatal stroke & $0.78(0.65-0.94)$ \\
\hline
\end{tabular}

positive benefit/adverse event ratio, they cautioned against use of statins in primary prevention patients thus: "below a $1 \%$ annual all-cause mortality risk or an annual CVD event rate of below $2 \%$ observed in the control groups in the trials considered here - [statin therapy] is not supported by the existing evidence". They further commented that there was insufficient demonstration of improvement in quality of life and cost-effectiveness associated with statin therapy.

Responses to these findings were immediate, strong, and largely opposed the authors' conclusions. The accompanying editorial ${ }^{36}$ cited a number of concerns. A major one revolved around the limitations inherent in uniting the diverse published data. Baigent, ${ }^{233}$ whose more complete work in the Cholesterol Treatment Trialists' Collaboration was not considered, vigorously challenged the conclusions, citing the significant benefits of statin therapy in relation to safety that the authors readily discussed, yet ignored. Other prominent researchers termed the study biased, inappropriately using data limitations that were irrelevant to the central issue. In addition, the conclusions were diametrically opposed to the growing evidence that statins are in fact underprescribed, rather than overprescribed, ${ }^{222}$ and that less stringent criteria for the earlier use of statins would reduce total risk burden and subsequent events. Not only does the philosophy in the Cochrane review ${ }^{298}$ disagree with current evidence-based guidelines, but also argues against the rationale behind many current programs and public health initiatives, including those advocated by WHO and disadvantaged countries, now exploring implementing the distribution of polypills. Lack of inclusion of all pertinent studies, the weights given to those considered, and subsequent interpretations were all criticized. Finally, it was said that adverse reactions, such as cognitive defects and depression, were given credence without adequate and critical examination of the evidence.

\section{Sources of controversy regarding statin use in primary prevention}

The American College of Cardiology, American Heart Association, National Cholesterol Education Program, NHLBI, and European Society of Cardiology incorporate statins in recommendations for both primary and secondary prevention. About $75 \%$ of patients who take statins do so for primary prevention. Presently the studies forming the basis for this prescribing are short, from 5-7 years' duration. The advice relies upon both scientific data and considered logical inference predicting that over a long period, cholesterol reduction will be reflected in improved mortality outcomes. Several researchers believe the use of 
statins over an extended period of time has underestimated potential. ${ }^{138,251,255,295,299,300}$

As mentioned, patients with CHD already have widespread and advanced disease, but in general, the atherogenic process is fairly intense by the time a diagnosis is made. It is likely that the beneficial effects from statin therapy, particularly mortality, take a longer period of time to demonstrate during the earlier stages of the disease, ie, during primary rather than secondary prevention. The chief benefit of statins in primary prevention is reflected in lower rates of nonfatal MI. Because mortality is low in such patients, the sensible interpretation is that the therapy was not begun sufficiently early, was not potent enough, or not continued long enough for mortality benefits to become significant. Similarly, one would not ordinarily expect, for instance, a striking reduction in mortality after 3.7 years when treating hypertension or smoking for primary prevention. The implication of the Ray and Taylor studies is that, for a mortality reduction, one should consider denying statins to patients until risk is high ( $\geq 20 \%$ 10-year FRS score), or the initial coronary event or diagnosis is made. This view is consistent with the NICE approach, ${ }^{283-285,301}$ but contrary to impressive data suggesting early and prolonged intervention is necessary. For the aforementioned reasons, the results of these meta-analyses will probably not be practice-changing. In summary, the absolute benefits of statins may be relatively small in a primary prevention population at low risk, and benefits are difficult to demonstrate short-term.

Given the economic constraints that exist in some countries, along with the political controversy surrounding health care delivery in the USA, the choice of medical services to be delivered now depends on factors other than science. Such issues may influence discussions in the academic literature. Most evidence indicates that populationbased use of statins is in fact cost-effective, but this is a function of population characteristics, cut-offs used, and the statin involved. ${ }^{302}$ The impact of generic atorvastatin was not considered in the Taylor study discussed above.

A large source of confusion about statin therapy concerns adverse reactions. There is a substantial amount of information circulating about side effects that is simply not evidence-based. Beliefs about the prevalence, severity, management, and threat of such reactions underpin reluctance to treat by practitioners and create unfounded fear in patients. Muscle aches and minor musculoskeletal discomfort are among the most common of human complaints, but when patients are taking statin drugs, a connection is assumed, commonly fueled by an inordinate number of inaccurate discussions or anecdotes on the internet. Pertinent data do suggest that: a) side effects are "underreported" in published trials, in part an artifact of low myotoxicity due to strict screening and run-in periods; b) the incidence of side effects do not approach what is believed by some practitioners and consumers, eg, rates of $\approx 50 \%$ mentioned on the internet, compared to the actual $\approx 10 \%$; c) life-threatening adverse reactions are extremely rare; d) most unwanted effects may be avoided and managed successfully; e) the incidence of intolerance and myotoxicity differs between statins; f) the benefits of statins far exceed those of adverse reactions, whereas other common drugs, such as aspirin, lack the same benefit/risk ratio in many settings. ${ }^{15,303-306}$

\section{Statins and primary prevention in the elderly: a special population}

Commenting upon a paper by Deedwania and coworkers in 2007, which reported that intensive statin therapy was not superior to a moderate approach in suppressing ischemia on monitor recordings in the elderly, ${ }^{307}$ Gotto advised judicious application of statins in the elderly population pending further data, ${ }^{308}$ which are now available.

Statins are underutilized in treating the elderly with cardiovascular risk, and a significant fraction of those individuals could benefit from statin therapy. Increasing age is a powerful risk factor, CHD is the most common cause of death in patients over 65 years, and over $80 \%$ of deaths due to cardiovascular disease occur in this population. The interaction of atypical presentations, multiple comorbidities, and polypharmacy may create uncertainty. Response to therapy is less predictable, while complications are more likely. Assigning causation, and prioritizing importance of pathophysiology from clinical and laboratory findings become difficult. When the benefit-to-risk ratio of treatment alternatives become unclear, caution prevails. With increasing age, relative risks of high LDL-C levels decline. Muscle and other side effects of statins often affect older patients' quality of life more than in the young. ${ }^{309}$ All may contribute to the low attainment of evidence-based targets in this population. ${ }^{310}$ Maroo et $\mathrm{al}^{311}$ revisited the issue by reviewing four studies in the elderly, and concluded that statins provided benefit in high-risk older patients, even though they might be more susceptible to unfavorable interactions. A large meta-analysis of ten randomized trials in individuals with risk factors but without a diagnosis of heart disease showed substantial benefit to the elderly in both risk factor reduction and improved survival. ${ }^{291}$ Wenger and Lewis, ${ }^{312}$ 
noting that a lack of awareness of potential benefits and perceived safety issues contribute to undertreatment, called for greater use of guideline-recommended LDL-C targets. Long et $\mathrm{al}^{313}$ provide a recent authoritative discussion of details in individual trials.

Pletcher and Coxson $^{314}$ discussed the possibility that $\mathrm{TC}$ and non-LDL-C were stronger risk factors in the young than the old, with relative risks of $0.44 / 1-\mathrm{mmol} / \mathrm{L}$ fall in TC at ages $40-49$ vs 0.72 at ages $60-69,{ }^{315}$ a relationship which they believed was also suggested in statin trials. ${ }^{233}$ Pletcher et $\mathrm{al}^{316}$ performed an analysis of age interaction of LDL-C and cardiovascular responses to statin therapy, and found that age may weaken statin effectiveness. Hayward, ${ }^{317}$ however, disagreed, citing the absence of age-dependent variation in responses to statins in primary prevention in a survey by NICE. ${ }^{283}$

Glynn and collaborators ${ }^{318}$ analyzed a subgroup of the JUPITER study of 5,695 persons over 70 years of age in the largest primary prevention statin study in this population. Compared to a cohort 50-69 years old, a greater proportion were women and hypertensive, with fewer smokers and overweight individuals. Reductions in LDL-C and CRP in the rosuvastatin-treated group were comparable to those in younger participants. The two groups combined demonstrated a $44 \%$ relative reduction in the primary end point of nonfatal MI, nonfatal stroke, hospitalization for unstable angina, arterial revascularization, or confirmed death from cardiovascular causes. Even though their response to rosuvastatin was significant, $70 \%$ of the elders studied had an FRS $>10 \%$ and $65 \%$ were hypertensive. How much additional risk was conferred by elevated CRP levels was uncertain, since CRP levels rise with age ${ }^{319}$ A large number of the subjects would have qualified for statin therapy using conventional guidelines. Therefore it was not surprising that in the older patients, the nonhypertensive subset did not benefit from rosuvastatin therapy. Since the absolute reduction in risk was greater in the $\geq 70$ year old group, the number needed to treat was 24 , compared to 36 in the group aged 50-69 years. Overall, the data confirmed that in higher-risk elderly patients, rosuvastatin should not be withheld for fear of adverse effects.

\section{Residual risk remains a source of concern}

In preventive cardiology, residual risk commonly refers to risk remaining after statin therapy has achieved LDL-C targets. However, residual risk also exists when multiple risk factors are being treated simultaneously. To be sure, traditional or "major" risk factors account for the greater portion of risk, and the population-attributable risk for each one are known. ${ }^{99}$ Non-lipid risk factors commonly coexist, and current practice guidelines recommend that all risk factors should be addressed for the best outcomes. Hence, a fuller definition of residual risk might be the vascular risk that persists after evidence-based targets are attained for dyslipidemia, hypertension, hyperglycemia, and inflammation.

\section{Lipid-mediated residual risk during statin therapy}

The average adult untreated LDL-C level in the USA is $\approx 130 \mathrm{mg} / \mathrm{dL}(3.4 \mathrm{mmol} / \mathrm{L})$. Half of the individuals with "normal" levels $\approx 100 \mathrm{mg} / \mathrm{dL}(2.6 \mathrm{mmol} / \mathrm{L})$ will have atherosclerosis by age $50 .{ }^{320}$ In the Pravastatin or Atorvastatin Evaluation and Infection Therapy (PROVE-IT), aggressive lowering of lipids used only prevented $\approx 20 \%$ of cardiac events. ${ }^{321}$ There is $\approx 30 \%$ relative risk reduction with aggressive statin therapy using current guidelines, resulting in about $0.5 \%$ lowering of major adverse cardiovascular events (MACE) per $\mathrm{mg} \%$ reduction in LDL-C, amounting to a $24 \%$ MACE reduction per each $1 \mathrm{mmol} / \mathrm{L}$ fall in LDL-C. ${ }^{234}$ This leaves a yearly ongoing incidence of $\mathrm{MACE}$ of $\approx 9 \%$ in such patients despite aggressive statin therapy. ${ }^{233} \mathrm{PROVE}^{2} \mathrm{IT}^{322}$ and a dual target analysis of JUPITER ${ }^{278}$ suggested that further reduction of LDL-C to $\approx 40 \mathrm{mg} / \mathrm{dL}$ may safely lower event rates even more. In the major well-known statin studies residual risk averages $65 \%-75 \%$, and in studies aggressively lowering LDL-C to $<100 \mathrm{mg} / \mathrm{dL}$, residual risk persists. For this reason, simply reducing LDL-C to lower levels is of value, but residual risk is still significant. Use of more potent agents, such as rosuvastatin, with somewhat greater ability to raise HDL-C, may also be of benefit. For instance, in early statin studies using pravastatin, residual risk was $\approx 76 \%$; use of rosuvastatin in the JUPITER trial was associated with a residual risk of $56 \%$, certainly an impressive improvement.

Using intravascular ultrasound, Bayturan et al found that in about $20 \%$ of patients treated intensively down to a mean LDL-C of $58.4 \mathrm{mg} / \mathrm{dL}(1.5 \mathrm{mmol} / \mathrm{L})$, plaque volume still increased. ${ }^{278}$

A portion of residual risk arises from atherogenic particles other than LDL-C, especially components of non-high-density lipoproteins (non-HDL). These include intermediate density lipoprotein, very low-density lipoprotein (VLDL), chylomicron and VLDL remnants, and lipoprotein (a). High triglyceride levels, low amounts of HDL-C, and defective HDL also contribute to risk. In the PROVE IT-TIMI 
22 trial, ${ }^{323}$ lower on-treatment $\mathrm{CV}$ risk was associated with $\mathrm{TG}<150 \mathrm{mg} / \mathrm{dL}$. Cardiovascular event rates are higher when HDL-C is low in statin-treated patients, ${ }^{233}$ while the additive risk of low HDL-C values is greater when LDL-C is low. ${ }^{324,325}$ The EPIC (European Prospective Investigation Into Cancer and Nutrition)-Norfolk study ${ }^{326}$ established that, independent of LDL-C levels, individual non-HDL-C $\geq 30 \mathrm{mg} / \mathrm{dl}$ higher than LDL-C predicted increased CHD risk, and triglyceride (TG) levels $>150 \mathrm{mg} / \mathrm{dL}$, or TC/HDL-C ratio $>5$ was associated with elevated risk for CHD. Carey et $\mathrm{al}^{327}$ conducted a hospital-based, case-control study in patients at optimal LDL-C levels and also found strong and synergistic effects of high TG and low HDL-C levels upon CHD risk in patients with optimal rather than higher LDL-C, even $<70 \mathrm{mg} / \mathrm{dL}$. Specifically, there was a rise of $\approx 20 \%$ in risk associated with a $23 \mathrm{mg} / \mathrm{dL}$ increment in $\mathrm{TG}$, and an increase in risk of $\approx 40 \%$ when HDL-C was $7.5 \mathrm{mg} / \mathrm{dL}$ lower. In patients who have achieved an LDL-C $\leq 70 \mathrm{mg} / \mathrm{dL}$, Núñez-Cortés et $\mathrm{al}^{328}$ estimated that an increase in HDL-C of $1 \mathrm{mg} / \mathrm{dL}$ would be expected to reduce the risk of subsequent MACE by $\approx 1.1 \%$.

While most of the risk associated with obesity is mediated through hypertension, dyslipidemia, and hypertension, obesity itself is an independent risk factor. The pathogenetic events leading to raised cardiovascular risk in overweight and obesity are complex, heavily researched, remain ill-understood, but extend beyond metabolic, hormonal, and cytokine dysregulation ${ }^{329-343}$ to include endothelial dysfunction, local pericardial fat accumulation, ${ }^{341,342}$ liver involvement, and adrenergic disturbances. ${ }^{315}$ Even when evidence-based targets are attained in all areas of traditional risk factors, residual risk persists. In diabetics, the risk is greater than is accounted for by the degree of separate risk factors. Inability to eliminate risk further by additional lowering of blood pressure, ${ }^{345}$ glucose, ${ }^{346}$ and through combination anti-lipid treatments ${ }^{347,348}$ may be explained by a complex biochemical web producing risk in diabetes. ${ }^{349}$ For these reasons, lifestyle modification and exercise remain fundamental cornerstones in the management of risk in these populations. In patients with diabetes and insulin-resistance syndromes, a "normal" baseline LDL-C sometimes creates reluctance to begin or intensify statin therapy, but in such patients with clearly elevated risk, benefits occur independently of initial LDL-C levels, and they should not be withheld. The sources of residual risk and management in patients with atherogenic dyslipidemia is further discussed by Hermans and Fruchart. ${ }^{350}$

\section{High-density lipoprotein (HDL)}

In general, a rise of $1 \mathrm{mg} / \mathrm{dL}$ in HDL-C is associated with a $3 \%$ lower CHD risk in women, and a $2 \%$ lower CHD risk in men. ${ }^{351}$ Epidemiological studies associate low HDL-C levels with an increase in the rates of myocardial infarction (MI), stroke, and mortality. High HDL-C levels ( $\geq 60 \mathrm{mg} / \mathrm{dL}$ ) are protective, whereas a low HDL-C level $(\leq 40 \mathrm{mg} / \mathrm{dL}$ in men, $\leq 50 \mathrm{mg} / \mathrm{dL}$ in women) is an independent risk factor for future cardiovascular events; some guidelines advocate raising low HDL-C levels, especially in high-risk patients. In general, raising HDL-C levels is considered beneficial for much of the population. ${ }^{241,352,353}$ NHANES 2003-2004 ${ }^{354}$ reported the mean HDL-C in the USA was $54.3 \mathrm{mg} / \mathrm{dL}$, and was low in $27 \%$ of all NHANES participants, and in $35 \%$ of NHANES subjects with CVD. Hence prevalence of low HDL-C is common, affecting about one-third of the American population, with an HDL-C $<20 \mathrm{mg} / \mathrm{dL}$ found in $\approx 0.5 \%$ of men and $\approx 0.25 \%$ of women. HDL-C levels depend upon genetic and environmental factors, with lifestyle choices, including the use of alcohol and tobacco, among the reversible factors. As a result of the rise in the prevalence of obesity, diabetes, and the metabolic syndrome, the mean HDL-C in patients presenting with an acute coronary event has fallen during the past 10 years to $38 \mathrm{mg} / \mathrm{dL} .{ }^{355}$

In 12,339 subjects without CHD at baseline in the ARIC study, ${ }^{356} 10$-year risk was lowest for those with LDL-C values in the lowest quintile (men, $95 \mathrm{mg} / \mathrm{dL}$, women, $88 \mathrm{mg} / \mathrm{dL}$ ) and lowest in the highest quintile for HDL-C (men, $62 \mathrm{mg} / \mathrm{dL}$, women, $81 \mathrm{mg} / \mathrm{dL}$ ). When adjusted for LDL-C, HDL-C, and $\mathrm{TG}$, each 1 standard deviation rise in HDL-C, $15 \mathrm{mg} / \mathrm{dL}$, was linked to relative CHD risks of 0.64 in men and 0.69 in women. The Emerging Risk Factors Collaboration, ${ }^{357}$ a meta-analysis with over 300,000 subjects without CHD at baseline, found that rates of CHD per 1000 person-years in the lowest and highest tertiles of baseline lipid distributions, respectively, were 2.6 and 6.2 with triglyceride, 6.4 and 2.4 with HDL-C, and 2.3 and 6.7 with non-HDL-C. On the other hand, in a meta-regression analysis that included 108 randomized trials of lipid reduction upon outcomes ${ }^{358}$ with about an equal number of participants at risk of cardiovascular events, simply raising the amount of HDL-C by $1.7 \mathrm{mg} / \mathrm{dL}(3.6 \%)$ had no effect upon CHD morbidity, CHD mortality, or total mortality after adjusting for the LDL-C levels.

In the Air Force/Texas Coronary Atherosclerosis Prevention Study (AFCAPS/TexCAPS), the ratio apolipoprotein B (apoB)/apolipoproteinA-I (apoA-I), discussed further below, predicted cardiovascular risk better than LDL-C or 
HDL-C. ${ }^{359}$ Of all available statins, rosuvastatin simultaneously produces the greatest increases in HDL-C and apoA-I, together with the best reductions in LDL-C and apoB. ${ }^{360,361}$ In the ASTEROID trial, ${ }^{241} \mathrm{HDL}-\mathrm{C}$ levels were inversely related to $\mathrm{CHD}$ progression during statin therapy as assessed using quantitative coronary angiography and using intravascular ultrasound (IVUS). ${ }^{351}$ Negi and Ballantyne ${ }^{362}$ plotted the change in percent diameter stenosis per year vs the ontreatment HDL-C level in an angiographic statin trial, which described a linear, monotone inverse relationship. The relative effects of rosuvastatin vs atorvastatin on atheroma volume, employing IVUS, is being compared in the Study of Coronary Atheroma by Intravascular Ultrasound: Effect of Rosuvastatin vs Atorvastatin (SATURN), now in progress. ${ }^{363}$

\section{HDL biology-function, quantity, and quality}

HDL is a mixture of heterogenous particles synthesized in the liver, jejunum, on the surfaces of macrophages, and in serum. They vary in size, density (between 1.063-1.21 g/mL), composition, surface charge, and function. HDL shape ranges from discoid to spherical, depending upon its lipid composition. The biology of HDL is more complex than that of LDL, and capable of slowing atherosclerosis through several mechanisms. The number of regulators - genetic polymorphisms affecting synthesis of apoproteins, receptors, enzymes that remodel lipoproteins, and inflammatory markers that determine HDL-C levels, maturation, and function - are staggering, but multiple steps and regulators simultaneously provide additional therapeutic opportunities. ${ }^{364}$ Over 75 different proteins are found within HDL populations that are concerned with lipid metabolism, complement activation, acute phase response; and protease inhibition. ${ }^{365}$ Within the total HDL-C weight, the subfractions function differently, each with a specific biological function ${ }^{366}$ which is partly dependent upon the triglyceride level. The complex proteasome and lipidosome of HDL particles, in constant flux, may alter individual HDL particle function as its composition changes. The most important protein component is apolipoprotein A-I (apoA-I), accounting for approximately $70 \%$ of HDL protein mass, with apoA-II comprising $15 \%$ to $20 \%$. Hence, apoA-I is sometimes considered a surrogate for HDL-C. Physically, apo-AI occupies about $85 \%$ of the surface of HDL, and it is believed that HDL size is modulated by a twisting motion of the apo-AI molecule. Cross-linking chemistry and mass spectrometry data suggest that apo-AI adopts a symmetrical cage-like structure to hold the lipid cargo. HDL interacts functionally with scavenger receptor class B type I (SR-BI), a cell surface glycoprotein, and the resulting SR-BI signaling is important in hepatocytes, endothelial cells, macrophages, and platelets. ${ }^{367}$

The most significant atheroprotective function of HDL is reverse cholesterol transport (RCT). Pre $\beta 1$-HDL, the lipiddepleted form of HDL, receives cholesterol from arterial plaque via macrophage ATP-binding cassette transporter ABCA1. In these enlarging particles, apoA-I accepts more cholesterol from the periphery, with the assistance of lipoprotein lipase and cholesteryl ester transfer protein (CETP), to become mature $\alpha 1$-HDL particles. ${ }^{368}$ Eventually cholesterol is returned to the liver or to apolipoprotein B (apoB)containing cholesterol-acceptor particles. ${ }^{369}$ ApoA-I in HDL plays a major role in RCT: underexpression of apoA-I is antiatherogenic, and small peptides that mimic some properties of apoA-I may impede atherosclerosis and improve HDL function, even without a rise in HDL-C levels. ${ }^{370}$ Raising apoA-I production also increases the the number of pre $\beta 1$ HDL particles and is antiatherogenic. Various functions of HDL are summarized in Table 7.

These properties collectively provide significant pleiotropic protection against atherothrombotic disease, not only by modulating lipids, but also through non-lipid antioxidative, antiinflammatory, and antithrombotic mechanisms. Rosuvastatin produces the greatest rise in HDL-C compared to other statins, and for this reason may be favored, particularly in women, or in patients who are overweight, smokers, or glucose intolerant, all of which depress HDL-C levels. Proof, however, of improvement in clinical outcomes when HDL-C is raised in these settings, is presently lacking, and awaits data from ongoing studies.

\section{“Dysfunctional” HDL}

Unfortunately, quite apart from the torcetrapib experience, higher HDL-C levels, as mentioned above, are not always associated with improved cardiovascular outcomes. ${ }^{377-381}$ When estrogen and progestins were administered in the Women's Health Initiative, ${ }^{382}$ HDL-C rose $7.3 \%$, but CHD risk increased by $29 \%$. During pro-oxidative and inflammatory states, including CHD, diabetes, metabolic syndrome, infections, surgery, active rheumatic conditions, chronic kidney disease, obstructive sleep apnea, and other diseases, HDL may become "dysfunctional". ${ }^{383}$ Rather than function as an antioxidant, it may even result in a pro-oxidant, pro-inflammatory phenotype; ${ }^{384,385} \mathrm{HDL}$ from patients with CHD may promote LDL oxidation. ${ }^{386} \mathrm{HDLs}$ from individuals 
Table 7 Some functions of high-density lipoprotein (HDL)

- Participation in reverse cholesterol transport of cholesterol, through the ATP-binding cassette (ABC) transporters and additional mechanisms, is the most important vasculoprotective function. Since peripheral cells cannot metabolize cholesterol, it will accumulate unless carried back to the liver for disposal. Heterogeneous HDL particle subfractions are continuously exchanging moieties and interacting with other lipoproteins, lipolytic enzymes (hepatic and endothelial lipases), and transfer proteins (lecithin:cholesteryl acyltransferase, phospholipid transfer protein). A major feature of HDL remodeling involves transfer of cholesteryl ester from cholesterol-rich HDL in exchange for triglycerides from apolipoprotein B (apoB)containing cholesterol-acceptor particles. ${ }^{371}$ While the atherosclerotic process is affected by cholesterol efflux capacity from cultured macrophage foam cells, this property may be independent of the HDL-C level, ${ }^{372}$ related more to its function, ie, quality, rather than quantity. ${ }^{373}$

- Protection and support of endothelium, through inhibition of monocyte chemotaxis, adhesion molecule expression, and enhanced nitric oxide production. ${ }^{365}$

- Prevention of LDL oxidation, involving two redox active methionine centers in apoA-I, paraoxonase-I (PON-I) and paraoxonase-3,374,375 as well as other component antioxidant enzymes contained in HDL, such as glutathione peroxidase, and platelet activating factor acetylhydrolase).

- Promotion of efflux of oxidized LDL from macrophages.

- Antiproliferative actions, particularly reducing apoptosis in macrophages.

- Anticoagulation, via inhibition of factors Va and VIla and promotion of urokinase-dependent fibrinolysis.

- Inhibition of platelet activation and aggregation, which protects the endothelium and inhibits thrombosis.

- Augmentation of prostacyclin synthesis through activation of cyclooxygenase-2.

- Stimulation of endothelial cell migration and recruitment of endothelial progenitor cells at sites of endothelial injury.

- Reduction of inflammatory mediator expression.

- Participation in the immune system, through component complement proteins. ${ }^{376}$

- Inhibition of atherogenic remnant particle production, by maintaining VLDL-triglyceride homeostasis.

- Promotion of glucose uptake and fatty acid oxidation, tempering any rise in insulin resistance, along with upregulation of pancreatic insulin secretion.

- Mediation of vascular protection ascribed to $17 \beta$-estradiol, the most potent endogenous estrogen, via enhanced HDL-induced endothelial nitric oxide synthase 3 activity to increase nitric oxide release.

recently diagnosed with CHD, but not yet treated with statins, have a unique protein cargo, compared to HDL found in healthy control subjects. ${ }^{365,387}$ There are many molecular mechanisms through which HDL may become dysfunctional, including changes in protein composition, change in antioxidant activity, overexpression of apoA-II, infectious processes and toxins, enzymatic modification of constituent HDL proteins and/or lipids, oxidation, interaction with inflammatory mediators, and others.

Common in vitro processes that may adversely affect the cholesterol efflux property of normal HDL are oxidation, preventing the maturation of HDL, advanced glycation of HDL, and chlorination and nitration of HDL by myoperoxidase (MPO). ApoA-I, the primary protein constituent of HDL, is a selective target for MPO-catalyzed nitration and chlorination in vivo. Such MPO-catalyzed oxidation of HDL and apoA-I selectively inhibits ABCA1-dependent cholesterol efflux from macrophages, which in turn promotes oxidation of LDL. ${ }^{38,388}$ Other processes during inflammation that may impair HDL function are increased HDL catabolism, displacement of ApoA-I from HDL by the acute phase reactant serum amyloid A (which also impairs activity of lecithin:cholesterol acyltransferase, paraoxonase, and Lp-PLA ${ }_{2}$, ${ }^{389}$ lower synthesis of apoA-I $\mathrm{I}^{390,391}$ and decreased activity of platelet-activating acetylhydrolase and lecithin:cholesterol acyltransferase (LCAT). ${ }^{392,393}$ During acute phase reactions beneficial proteins in HDL particles other than ApoA-I may also be may exchanged for fibrinogen or soluble lipoprotein associated phospholipase $\mathrm{A}_{2}$. In addition, changes in proteins that are not constituents of HDL, but are concerned with HDL metabolism, such as SR-BI, may be proatherogenic even when HDL-C is increased. ${ }^{394}$ Finally, patients with polymorphisms in the promoter for hepatic lipase have higher HDL-C levels but raised risk for $\mathrm{CHD} .{ }^{376}$ Hence individual genetic mutations and metabolic status may result in critical loss of HDL function. Other genetic variations in which there may be a divergence of HDL-C levels and the anticipated inverse CHD risk are discussed by Tall et al. ${ }^{395}$ Treatment of patients with nongenetic dysfunctional HDL with statins ${ }^{396}$ or statins combined with niacin ${ }^{397}$ may partially reverse the impairment in HDL function. Sorrentino et al ${ }^{398}$ reported that HDL from diabetics was deficient in their ability to promote endothelial progenitor cell-dependent endothelial repair, increase endothelial nitric oxide expression, and produce endothelium-dependent relaxation. These defects were associated with raised HDL lipid peroxidation and MPO content, and could be improved with niacin therapy. Exercise is a frequently-overlooked, effective modality to partially repair defective HDL and raise HDL-C levels.

Interestingly, CRP and MPO have been linked together as predictors of prognosis in a number of cardiovascular outcome studies. ${ }^{398}$ CRP has also been shown to stimulate MPO release in human polymorphonuclear cells and 
monocytes in vivo, and may have significance in patients with acute coronary syndromes. ${ }^{400}$

With insulin resistance, the metabolic syndrome, acute inflammation, and in hypertriglyceridemia, more triglyceride-rich HDL is produced as a result of enhanced CETP activity. A number of events may lead to lower HDL-C and HDL-P, including remodeling of triglycerideenriched HDL by hepatic lipase, resulting in enhanced binding, internalization, and degradation of HDL, as well as instability of these moieties with loosely-bound apoA-I, without major roles of either SR-BI or proteoglycans. ${ }^{401-403}$ Moreover, the triglyceride-rich HDL uptake by SR-BI may be deficient, reducing the effectiveness of reverse cholesterol transport. ${ }^{366}$ In diabetes, the typical lipid phenotype includes small, dense HDL particles, enriched with triglycerides and depleted of cholesteryl esters, which have lost $20 \%-50 \%$ of their antioxidative capacity. ${ }^{404}$ This finding correlates with elevated levels of 8-isoprostanes, robust markers of oxidative stress. ${ }^{405,406}$ Since patients with metabolic syndrome will not typically progress to overt diabetes unless there is pancreatic beta-cell failure, the recently-described potential role of deficient and defective HDL in the future loss of insulin secretion by beta cells through $\mathrm{ABC}$ transporters is of importance. ${ }^{407}$ In addition, insulin resistance may increase HDL catabolism and renal excretion, adding to the difficulty of raising HDL-C levels in such patients.

\section{$\mathrm{HDL}$ as a component of the immune system}

Proteomic analysis of $\mathrm{HDL}^{365}$ has identified more proteins involved with acute-phase response than are associated with lipid metabolism, consistent with the view that a major role of HDL is to inhibit inflammation. Moreover, a number of proteins within HDL regulate complement activation, which is known to have a role in atherogenesis. ${ }^{408}$ Potential triggers of complement activation within atherosclerotic lesions include immune complexes, CRP, oxidized and glycated lipoproteins, apoptotic cells, cholesterol crystals, and possibly dysfunctional HDL. ${ }^{409}$ Circumstantial evidence supports the hypothesis that HDL not only is responsible for cholesterol trafficking, but also plays a part in the immune system protecting against infection. Such a system envisions HDL assisting in the removal of apoptotic cells from inflamed and/or infected sites. Thus, in addition to the removal of cholesterol from macrophages, the additional exchange of proteins and lipid moieties between HDL and macrophages may regulate inflammation. Exposure of macrophages to bacterial endotoxin lipopolysaccharide (LPS) downregulates the transporters $\mathrm{ABCA} 1$ and $\mathrm{ABCG} 1$, thereby suppressing their ability to efflux cholesterol. ${ }^{410,411}$ Any inflammatory mediators that use the same signaling pathways as LPS would inhibit reverse cholesterol transport. Direct proof was provided by administering LPS to human volunteers, which resulted in stimulation of acute phase reactants serum amyloid A and CRP. These changes were accompanied by remodeling of HDL, which became less able to accept cholesterol. ${ }^{409}$ Further confirmation came from septic patients, in whom inflammation reduced the ability of HDL to accept cholesterol by $73 \%$ as compared with controls. ${ }^{412}$ This proposal also fits with the larger notion that lipids are capable of activating circulating immune cells which may contribute to the pathogenesis of atherosclerosis. ${ }^{413}$

\section{Heritability of HDL-C levels}

About $40 \%-60 \%$ of HDL-C levels are heritable. ${ }^{414}$ Aside from the $\approx 40$ genetic polymorphisms in the APOAl gene which may contribute to variation in HDL function, genetic variation in genes encoding the many other substances involved in HDL metabolism, particularly CETP, appears to be clinically relevant. Genome-wide association studies demonstrate the strongest associations with HDL-C are found among CETP single nucleotide polymorphisms. For instance, the $B 2$ allele of the TaqIB polymorphism of CETP may be associated with recurrent cardiovascular events. ${ }^{415}$ The strength of CETP gene polymorphisms upon HDL-C is also uninfluenced, at least in high risk patients, by dietary interactions, eg, the Mediterranean diet, or with obesity, smoking, diabetes, or alcohol use. ${ }^{380}$ Although dysfunctional HDL may exist at all HDL-C levels, the association of high HDL-C with high CRP levels, especially in post-infarction patients, may be characterized by larger HDL particles, higher apoA-I and serum amyloid A levels, and suggests that further evaluation of HDL quality is warranted. ${ }^{414}$ The full clinical significance of dysfunctional HDL awaits greater use of a relatively new cell-free laboratory evaluation of HDL function. ${ }^{393,416}$

An interesting application begins with an analysis from the INTERHEART study, which reported that South Asians have an unusually high prevalence of low HDL-C levels. ${ }^{416}$ In South Asian immigrants, in whom metabolic syndrome is frequent, conventional risk factors, insulin resistance, and components of the metabolic syndrome, are insufficient to account for their raised CHD risk. ${ }^{418}$ Using CIMT and a novel cell free assay and HDL inflammatory Index, Dodani et $\mathrm{al}^{419}$ found that $70 \%$ of south Asian immigrants with subclinical CHD had dysfunctional HDL. It is hypothesized that a unique combination of genetic predisposition, high carbohydrate intake, lack of exercise, tobacco use, and low birth weight due to maternal malnutrition 
suppresses the activity of $\Delta^{6}$ and $\Delta^{5}$ desaturases and lowers the levels of anti-inflammatory essential fatty acids in South Asians. ${ }^{420}$

Comprehensive discussions about HDL biogenesis, life cycle and function, are available elsewhere in greater detail. $^{364,382,403-406,421-425}$

\section{Potential of HDL modification to lower risk}

Interest in raising HDL-C levels, or improving HDL function, continues in the ongoing search for methods to close the residual "risk gap". ${ }^{403,424,426}$ The most effective available agent is niacin, but practical problems with flushing limiting the use of this agent are well-known. The failure of proatherogenic cholesteryl ester transfer protein (CEPT) inhibitor torcetrapib, which produced a $70.3 \%$ rise in HDL-C levels, but an increase in CHD of $21 \%$, was due in large part to off-target toxicity characterized by aldosterone-associated hypertension, hypokalemia, and elevations in serum bicarbonate levels. ${ }^{427-429}$ However, that disappointment has recently been replaced by some optimism. A preliminary report of the study Determining the Efficacy and Tolerability of CETP Inhibition with Anacetrapib (DEFINE) indicates that anacetrapib, another CETP inhibitor, produces an outstanding $138 \%$ rise in HDL-C levels, a 39.8\% reduction in LDL-C, does not raise aldosterone levels, and may be associated with improved outcomes..$^{430-435}$ The beneficial lipid actions appear to be additive with those of statin drugs. CETP mediates exchange of lipids between HDL particles and other lipoprotein fractions. It remains to be shown whether the HDL produced by anacetrapib is biologically normal, cardiovascular events will be reduced, and safety will be demonstrated in a large randomized trial, which is now under way. ${ }^{430}$ Dalcetrapib is a second nontoxic CETP inhibitor under investigation with potential to raise HDL-C and lower LDL-C.

An additional approach involves stimulating the endogenous production of apoA-I in patients with CHD in order to raise pre $\beta 1-H D L$ particle number and enhance RCT. A new oral agent, RVX-208, selectively induces nuclear transporter factors to induce hepatic ApoA-I synthesis, and has been shown to increase blood levels of both pre $\beta 1-\mathrm{HDL}$ and mature $\alpha 1$-HDL. In the first report from the ASSERT (ApoA-I Synthesis Stimulation Evaluation in Patients Requiring Treatment for Coronary Artery Disease) study, ${ }^{436}$ RVX-208 therapy raised apoA-I and HDL-C levels modestly, up to $5.6 \%$ and $3.2 \%-8.3 \%$ respectively, accompanied by an $11.1 \%$ to $21.1 \%$ rise in large HDL particles, actually less than is associated with niacin therapy. Unfortunately there were also significant increases in hepatic transaminase levels, which may limit the future of this particular agent. ${ }^{437}$

While an additive effect of maximizing HDL-C levels along with reductions in LDL-C has been appreciated for over a decade, ${ }^{438}$ the real potential of combining statins with other agents remains unknown. A beneficial effect upon atheroma burden was suggested in ASTEROID AS $^{230}$ and upon CIMT in ARBITER 6-HALTS ${ }^{439}$ when using niacin.

Recently the AIM-HIGH trial ${ }^{440}$ was stopped early by the NHLBI after 32 months. In this study, 3414 patients with cardiovascular disease, low HDL-C and high TG levels were given either simvistatin and a placebo, or simvistatin in adjusted doses and extended-release niacin 1500-2000 mg, with 515 of the patients in the treatment cohort receiving ezetimibe $10 \mathrm{mg}$ if needed, to achieve LDL-C levels of 40 to $80 \mathrm{mg} / \mathrm{dL}$. Although niacin lowered TG and raised HDL-C levels as expected, there was no effect on a composite endpoint of fatal or nonfatal MI, stroke, hospitalization for acute coronary syndrome, or need for revascularization procedures. A small, unexplained increase in the rate of ischemic strokes in the niacin group also played a part in the decision to halt the study. It is important to note that the patient population had well-controlled on-treatment LDL-C levels. Generalizing these findings to other patient groups, particularly to those with higher LDL-C levels commonly encountered, is presently premature. Full interpretation awaits analysis and publication of the AIM-HIGH study data. Hopefully, the results of the more definitive HPS2-THRIVE study ${ }^{441}$ now in progress, involving 25,673 participants, will help answer some of the many questions raised.

However, these disappointing results suggest, as has been noted previously, that beneficial changes in surrogates such as risk factors do not necessarily mean improved outcomes, and now have significant implications for future drug investigations. In addition, the FDA currently approves new drugs based upon biomarker end points.

Despite the well-documented association of high HDL-C levels with cardiovascular protection, and low HDL-C values with poorer outcomes in atherothrombotic disease, pharmacological manipulation of global HDL-C levels is complex, probably not sufficiently specific with respect to the HDL molecule, and differs with each agent used.

\section{Other lipid targets: non-HDL-C and lipid fractions}

Compared to estimates of LDL-C, does measurement of apolipoproteinB, total cholesterol/HDL, apoB/apoA-1, 
or non-HDL-cholesterol (non-HDL-C, total cholesterol HDL-C), offer any advantage as a predictor, and as a therapeutic target? Early Framingham data showed LDL-C was predictive, but total cholesterol/HDL-C ${ }^{442}$ and apoB/ apoA-1 are equal, if not better, predictors. ${ }^{443}$ Ratios show a stronger correlation with cardiovascular events than does LDL-C. ${ }^{99,444,445}$ Since over half of all such events occur in apparently healthy persons without abnormal LDL-C levels, thinking beyond LDL-C has been a recent goal in order to improve both predictive ability and reduce residual risk. The lay press frequently mentions that up to $77 \%$ of patients with cardiac events have normal cholesterol levels, to which there is no satisfactory reply. Moreover, the relatively high proportion of statin-treated patients hospitalized for new events, even though a significant number of them are already at LDL-C goals, is a further embarrassment. ${ }^{355}$

The estimated LDL-C derived from the Friedewald formula not only introduces error, and does not reflect all atherogenic particles, but the need for a fasting sample is inconvenient. Without the need to fast, non-HDL-C is easily derived from the prevalent lipid profile.

Non-HDL-C is strongly associated with cardiovascular events $^{446,447}$ and is sometimes considered a proxy for apoB. When calculated from the standard lipid profile, it measures the amount of cholesterol contained in all atherogenic lipoproteins (excluding any proatherogenic HDL) - LDL, IDL, VLDL, and Lp(a). On the other hand, apoB reflects the number of circulating atherogenic particles, and is expressed in $\mathrm{mg} / \mathrm{dL}$. LDL particle number (LDL-P) is the number of LDL particles per liter of plasma, expressed in $\mathrm{nmol} / \mathrm{L}$. In healthy people non-HDL-C may equal apoB and LDL-P for accuracy in risk assessment. ${ }^{448,449}$ However, when HDL levels are low, triglyceride values are high, and in patients with diabetes or metabolic syndrome, LDL-P and apoB are much better indices of cardiovascular risk. ${ }^{359,440-452}$ Compared to the 1970 s and 1980s, an increasingly greater proportion of patients being evaluated are overweight, have higher triglyceride (TG) levels and small, dense LDL particles. With an abundance of triglycerides and TG-rich particles, these patients overproduce VLDL in the liver, which accounts for the increase in small, dense LDL and their low concentrations of HDL-C. ${ }^{453,403}$ As the number of individuals with visceral adiposity in the population - now at 53\% - increases, the accuracy of standard lipid profiles to predict risk diminishes, particularly in overt diabetics, since the discordance between LDL particle number and LDL-C enlarges as triglyceride values rise above $\approx 160 \mathrm{mg} / \mathrm{dL}$. An American Diabetes Association/ American College of Cardiology consensus statement ${ }^{454}$ considered this discordance and some limitations of the method. A more recent position statement by the American Association for Clinical Chemistry favored LDL-P as an accurate indicator of risk, and reviewed the advantages of monitoring particle number in order to reduce residual risk. ${ }^{455}$ Tests for LDL-P are FDA approved and their predictive ability was confirmed in the MESA study. ${ }^{456}$ These statements reflect that risk is better captured by apoB or LDL-P than by non-HDL-C, which itself may be discordant with apoB in about one-third of patients, and many lipidologists believe non-HDL-C is superior to LDL-C. It is important to note that the effect of individual statins upon lipid subfractions may differ, ${ }^{457}$ as may the relative effect upon LDL-P. In 318 patients with dyslipidemia and the metabolic syndrome, for instance, rosuvastatin was found to be superior to atorvastatin in lowering LDL-P. ${ }^{458}$ Further discussion is available in the recent contribution by Dayspring et al. ${ }^{459}$

The NCEP-ATP III ${ }^{153}$ presently recommends LDL-C as the primary target to be monitored, but after the LDL-C goal is reached, and if triglyceride levels are $\geq 200 \mathrm{mg} / \mathrm{dL}$, non-HDL-C is set as a secondary goal at $30 \mathrm{mg} / \mathrm{dL}$ higher than the LDL goal. The fourth Joint European Societies Guidelines $^{31}$ estimates risk of fatal cardiovascular events using SCORE, ${ }^{150}$ which is similar to Framingham but uses total cholesterol/HDL-C as the primary target. The Joint British Societies (JBS 2) guidelines ${ }^{293}$ uses similar criteria and the total cholesterol/HDL-C, to estimate 10 -year risk, but employs LDL-C treatment targets.

Using Framingham data, Liu et $\mathrm{al}^{460}$ found that VLDL-C was a significant predictor of cardiovascular risk, and that non-HDL-C was superior to LDL-C in predicting risk. In a large study of healthy Japanese men and women, the total cholesterol/HDL ratio best reflected long-term changes in lipid risk with the least within-person variation when compared to LDL-C. ${ }^{460}$ The superiority of measuring ratios of pro- to anti-atherogenic lipoproteins with respect to errors was also supported by Glasziou et al. ${ }^{461}$ An analysis of INTERHEART data found that non-fasting apoB/apoA-I was superior to other cholesterol ratios for estimation of the risk of myocardial infarction for all ethnic groups, ages, and in both sexes. ${ }^{463}$

The causal role of elevated lipoprotein(a) [Lp(a)] in premature cardiovascular disease has also been of interest. Presently $\mathrm{Lp}(\mathrm{a})$ elevations are probably not being given sufficient attention, and many are missed in routine lipid 
measurements, yet its relationship with CHD is robust and specific. The EAS Consensus Panel has critically reevaluated $\mathrm{Lp}$ (a) as a risk factor, and supported screening patients at intermediate or high CHD risk with premature CHD, familial hypercholesterolemia, a family history of premature CVD and/or elevated Lp(a), recurrent CHD despite statin treatment, $\geq 3 \% 10$-year risk of fatal CVD according to European guidelines, and/or $\geq 10 \%$ Framingham risk. ${ }^{464}$

In summary, LDL-C has limitations which are well recognized. ApoB is a better predictor than LDL-C, ${ }^{465}$ is not generally available, and has not been embraced clinically. Non-HDL-C, a proxy for apoB, measures the cholesterol content within atherogenic lipoproteins and is easily derived from standard lipid profiles, but may not reflect the full residual risk when particle number is discordant. Non-HDL-C is incorporated in several guidelines. LDL-P is the most accurate when predicting risk, is only available through independent laboratories, and reimbursement is irregular. ${ }^{452,466}$ Targets for the highest risk patients are LDL-C $<70 \mathrm{mg} / \mathrm{dL}$, non-HDL-C $<100 \mathrm{mg}$ / $\mathrm{dL}$, apoB $<80 \mathrm{mg} / \mathrm{dL},{ }^{454}$ and perhaps LDL-P $\leq$ 1000-1100 nmol/L.

\section{Risk and the significance of CRP and inflammation in prevention and pathogenesis}

Epidemiological, pathological, clinical, and imaging studies have constructed an evolving model of atherothrombotic disease, spanning the period from biochemical and physical triggering of endothelial dysfunction to rupture of a vulnerable plaque. Although atherothrombosis was once considered to consist of simple lipid and "plumbing" problems, a unifying concept of the role inflammation is now supported by considerable data in both the clinical and preclinical sciences. The numerous beneficial anti-inflammatory pleiotropic actions of statins and results of the JUPITER study suggest inflammation matters clinically. ${ }^{467-472}$

In a number of conditions and processes, including chemical injury, hypercholesterolemia, hypertension, endothelial dysfunction, cytokine stimulation, oxidative stress, and others, trapping of chemically modified LDL occurs within the arterial wall. ${ }^{473}$ Inflammatory monocyte recruitment, ${ }^{474}$ under the influence of cytokines and other protein mediators, leads to the expression of scavenger receptors for altered LDL and the formation of foam cells. ${ }^{467} \mathrm{C}$-reactive protein is a biomarker of inflammation, with hepatic expression driven by interleukin- 6 (IL-6), the "messenger" cytokine (notifying and activating the immune system after tissue injury). High CRP levels are closely associated not only with infections but with vascular disease, cancer, and autoimmunity. Evidence underpinning the close associations of CRP levels with vascular disease and its predictive value are the result of the accrual of knowledge over a long period of time by many investigators. ${ }^{15}$ High-sensitivity C-reactive protein (CRP) is able to discriminate levels of the protein at concentrations far below the greater variations associated with generalized inflammation. There is evidence that CRP concentrations reflect aspects of inflammation related to lifestyle, such as visceral obesity, ${ }^{475,476}$ and the metabolic syndrome, ${ }^{477,478}$ lack of physical activity, ${ }^{479}$ vegetable and fruit consumption, ${ }^{480}$ omega-3 fatty acid ingestion, ${ }^{481}$ and alcohol intake, ${ }^{482,483}$ all of which may not be fully captured by other risk markers, such as LDL. In fact, despite great attention to the genetic variations influencing CRP levels, lifestyle is actually the more significant determinant. ${ }^{484}$ Further, CRP levels are associated with all 7 health behaviors and factors that were cited by the AHA as components of ideal cardiovascular health (Table 2 ). ${ }^{2}$

The associations among visceral adiposity, diabetes, metabolic syndrome and inflammatory markers, $341,485,483$ as well as the relationship between the first three entities with elevated $\mathrm{CRP},{ }^{484}$ are well-known. The link between the metabolic syndrome and atherosclerosis involves elements other than insulin resistance, and CRP levels correlate with both the diagnosis of metabolic syndrome and the number of risk components. ${ }^{488}$ Adipose tissue releases IL-6 which stimulates CRP synthesis, but is also a significant source of CRP itself. Weight loss in obese women lowers CRP and raises adiponectin concentrations. ${ }^{489} \mathrm{CRP}$ correlates with insulin levels, ${ }^{490}$ and the Mediterranean diet lowers insulin resistance and CRP levels. ${ }^{488}$ The Look AHEAD (Action for Health on Diabetes) study recently reported on a large cohort of overweight diabetic women whose CRP levels fell markedly in response to intensive lifestyle intervention resulting in weight loss over a 1 year period. ${ }^{492}$

While elevations of IL- 6 and CRP are associated with illnesses that are expected to shorten life, low values, while not a guarantee of freedom from diabetes or CHD, may generally reflect better health. The Rancho Bernardo Study ${ }^{493}$ found that higher concentrations of these inflammatory markers predicted shorter survival time and reduced lifespan among older men. In addition, in various clinical situations, CRP levels correlate with mortality ${ }^{494,495}$ and survival, ${ }^{496}$ with strong predictive ability in many cardiovascular scenarios. ${ }^{497-503}$ In the Emerging Risk Factors Collaboration, ${ }^{497}$ a meta-analysis of 54 prospective studies, CRP correlated better with future vascular events than either blood pressure or cholesterol. 
Although the prognostic value of CRP in a broad population of patients at high risk for ischemic events is accepted, clinical utility of using CRP in treatment remains debated. ${ }^{504}$

CRP, as a pattern recognition molecule capable of activating complement, functions as a regulator in the innate immune system, the latter increasingly recognized as a participant in atherosclerosis. ${ }^{471,472,474,505,506}$ Oxidized LDL and oxidized phospholipids on surfaces of apoptotic cells are recognized by macrophage scavenger receptors, have proinflammatory and proatherogenic properties, ${ }^{507}$ and CRP binds to both through recognition of the phosphorylcholine moiety in oxidized phospholipids. ${ }^{508}$ The innate immune system appears to prime normal protective $\mathrm{T}$ cell-mediated immunity, ${ }^{509}$ which is involved in the inflammation associated with the metabolic syndrome ${ }^{510}$ and hypertension, ${ }^{511}$ two conditions in which CRP levels are frequently elevated. Clinically, the involvement of inflammation may explain the correlation of CRP elevations and early atherosclerosis detected by CIMT. ${ }^{512,513}$

In addition to inducing release of proinflammatory cytokines from monocytes, upregulating NADPH oxidase activity, and promoting endothelial dysfunction, CRP appears to have a role in priming differentiation of human monocytes toward a proinflammatory M1 phenotype, a critical event in the pathogenesis of atherosclerosis. Transformation of monocytes, or M1 polarization, may be regarded as an on-off switch in the balance between pro- and anti-inflammatory processes, and lead to macrophage maturation, further expression of inflammatory cytokines, and tissue destruction. Macrophage infiltration of adipose tissue in obese animal models and humans is associated with both an absolute rise in the number of M1 polarized macrophages and reduced sensitivity to insulin. M1 monocytes also infiltrate atherosclerotic lesions.

Recognition of modified extracellular matrix proteins by the innate immune system results in collateral blood vessel remodeling to accept additional blood flow. The encounter of agonists and toll-like receptors (TLR), another class of pattern recognition receptors, specifically TLR2 and TLR4 on monocytes and extracellular matrix fragments, leads to inflammation through the activation of the nuclear factor kappa B and interferon response factor pathways, in turn increasing the expression of proinflammatory cytokines, chemokines, matrix metalloproteinases, interferons, growth factors, and other molecules involved in arteriogenesis. There is a dynamic interaction between reduced blood flow, modified extracellular matrix proteins, and collateral vessel growth/vascular remodeling, in which TLR, monocytes and T-lymphocytes are involved. ${ }^{514,515}$
The recent recognition of the important and extensive role of the innate immune system in arteriogenesis, the increase in diameter of preexisting arteriolar connections, arterial remodeling during ischemia and atherosclerosis adds yet another dimension to pathogenesis of the disease. ${ }^{516}$ The full role of this process as a mechanism which protects against ischemic injury has yet to be determined.

\section{Strategies proposed to improve cardiovascular risk reduction}

The concept of risk factors, introduced by the original Framingham investigators in 1961, essentially established preventive cardiology. Risk factors are now accepted antecedents of atherosclerosis whose levels predict subsequent cardiovascular events and are targets for therapy. The current approach to cardiovascular risk screening is summarized in a state-of-the-art paper by Berger and associates. ${ }^{52}$

Within the past few years there has been a reevaluation of reducing risk in the general population, and the central unanswered question is: how can people who will eventually have cardiovascular events be identified and their risk lowered? There is no ideal or "gold-standard" risk equation for assessment, nor a drug-response equation for treatment. Current issues in primary prevention of CHD include the long incubation period; methods of evaluating risk in the population; population-based vs individual risk-based approaches; role and refinement of global risk factor scores; choice and merits of nontraditional risk factors; multiple biomarker panels; imaging techniques in evaluation and ongoing therapy; value of advanced lipid testing; weights given to traditional risk factors, cutoff values and treatment targets, particularly LDL goals in guidelines; use of statins in primary prevention; reasons for low patient adherence with evidence-based therapies; causes of "clinical inertia" and lack of physician compliance with guidelines; and the etiologies, extent, and minimization of residual risk. Within this period, there have been several suggestions based upon models, proposals, and clinical protocols contributing to the dialog, enumerated below with additional commentary.

\section{Encourage population-based interventions emphasizing primordial prevention}

Primordial prevention is the unchallenged method of choice for risk reduction, far more efficient than pharmacological intervention. A long tradition of epidemiological data has accumulated since the pioneering report of de Lorgeril 
et al concerning the Mediterranean $\operatorname{diet}^{517}$ and the Lyon Diet Heart Study. ${ }^{518}$ About that same time, Stamler and coworkers $^{519}$ published a series of 2 cohorts totaling 366,000 participants and reported that just 4 favorable risk factors (BP $<120 / 80 \mathrm{~mm} \mathrm{Hg}, \mathrm{TC}<200 \mathrm{mg} / \mathrm{dL}$, abstinence from tobacco, and no diabetes) was associated with a 72\%-92\% lowered cardiovascular mortality and an additional 5.8-9.5 years of life. Since then, there have been a great number of ongoing investigations and exceptional contributions affirming the favorable impact of the Mediterranean diet pattern and physical activity upon all-cause longevity, cardiometabolic, and other chronic diseases. ${ }^{520-523}$ The evidence supporting the effectiveness of lifestyle modification upon cardiovascular outcomes is summarized elsewhere ${ }^{2,11}$ and continues to amass. ${ }^{40,524}$

While primordial prevention is most desireable, ${ }^{177,525}$ social and political barriers are considerable, and current individual resistance to major behavioral modification is complex and not well understood. Perhaps the milieu of modern life has de-emphasized personal responsibility - or made it so difficult - for so long that the public now dismisses attempts to reverse these concepts as impractical, imposing, irrelevant, or unworthy. The message that medical care is unlimited and uniformly successful at all stages of disease may also unwittingly reduce motivation for personal health ownership. It would therefore appear that public re-education is fundamental for further progress, rather than a wasteful endeavor.

At the same time, it has been suggested that continued attempts to reduce cardiovascular risk without the addition of population-wide prevention is destined to fail. ${ }^{526}$ Plainly, furnishing entire populations with unlimited scans, statins, stents, and surgery is not the best answer. For this reason, and because it is premature to declare risk reduction programs a failure without further data or better alternatives, interest in refining and continuing comprehensive primordial prevention, including education and counseling, continues. A combined, multipronged, intensive approach to cardiovascular risk reduction using many techniques will be necessary.

\section{Change the lipoprotein or lipid fraction that is monitored and targeted}

As discussed above, non-HDL-C measures all atherogenic lipoproteins which contain apolipoprotein B, including LDL-C, very low-density lipoprotein cholesterol (VLDL-C), intermediate-density lipoprotein cholesterol (IDL-C), lipoprotein(a), chylomicrons, and chylomicron remnants. Non-HDL-C provides a more complete measure of atherogenic particles than LDL-C and is believed to be superior in capturing residual risk and ability to predict cardiovascular events. Evidence now indicates that monitoring and targeting non-HDL-C can better predict cardiovascular events than use of LDL-C, $326,446,527,528$ with up to twice the yield. ${ }^{529}$ Advanced lipid testing may identify abnormalities in small, dense LDL particles, LDL-P, HDL2[b], and Lp(a) fractions in a surprising number of patients. Ideally, all lipid pathology should be addressed to minimize cardiovascular events.

\section{Use "lifetime risk" rather than global I0-year risk scores, and start treatment much earlier}

Estimates of lifetime risk assessment offer an important tool which may be used in conjunction with 10 -year risk. With $56 \%$ of American adults scoring a low 10-year, but a high lifetime risk, this issue has received increasing consideration. ${ }^{213}$ The difference becomes of particular concern in both young and asymptomatic people. ${ }^{185,530}$ Lifetime risk may be estimated after Lloyd-Jones ${ }^{27}$ or as a 30-year Framingham risk that accounts for competing risks. ${ }^{531}$ In the JUPITER study of rosuvastatin in primary prevention, about half of the participants had a 10-year FRS $<10 \%$, but a significant number benefited when their LDL-C was lowered from a mean of $108 \mathrm{mg} / \mathrm{dL}$ to a mean treated value of $55 \mathrm{mg} / \mathrm{dL}$, reflecting the degree of unrecognized cardiovascular risk in an asymptomatic population. ${ }^{532}$

Using lifetime risk, beginning treatment early, and continuing therapy over an extended period, matches the timing of treatment to the time of disease progression, which is amply supported by newer data concerning pathogenesis of the disease.

\section{Give statins to intermediate-risk individuals guided by CRP - dual targeting using JUPITER criteria}

The JUPITER study ${ }^{532}$ involved 17,802 individuals with $\mathrm{LDL}<130 \mathrm{mg} / \mathrm{dL}$ and $\mathrm{CRP} \geq 2 \mathrm{mg} / \mathrm{L}$, free from diagnosed cardiovascular disease or diabetes, and included women, minorities, and the elderly. The mean LDL-C was $\approx 100 \mathrm{mg} / \mathrm{dL}$, and the average FRS was $11.6 \%$. Treatment with rosuvastatin $20 \mathrm{mg}$, compared to placebo, was associated with a statistically significant $54 \%$ reduction in myocardial infarction, a $47 \%$ reduction in need for angioplasty or bypass surgery, a $48 \%$ reduction in stroke, a $43 \%$ reduction in venous thrombosis, and a $20 \%$ reduction in all-cause mortality. The JUPITER study group concluded that primary prevention patients 
with high CRP values were at greater risk despite acceptable LDL-C levels and low Framingham Risk Scores, and such individuals benefited from rosuvastatin therapy. The 5-year number to treat was 25 , which compared favorably with other primary prevention methods, such as hypertension.

JUPITER definitively established the efficacy of rosuvastatin in primary prevention. ${ }^{532}$ The controversy surrounding the JUPITER trial is discussed elsewhere. ${ }^{15}$ Proponents and critics agree that the benefits of rosuvastatin in JUPITER-eligible participants, however, were real. The US Food and Drug Administration approved new indications for rosuvastatin to include asymptomatic JUPITER-eligible individuals with 1 additional risk factor. The Canadian Cardiovascular Society guidelines recommend testing for CRP, and using statins in persons with low LDL-C and high CRP levels at intermediate risk. The joint European Society of Hypertension/European Society of Cardiology Guidelines suggested CRP measurements be included in the assessment of risk in hypertensive patients. ${ }^{533}$ In a recent post hoc analysis of JUPITER requested by European health authorities, ${ }^{534}$ patients with an estimated SCORE risk $\geq 5 \%$ or FRS score $>20 \%$ had significant reductions of $43 \%-53 \%$ reduction in the risk of MI, stroke, or cardiovascular death when treated with rosuvastatin, compared with those treated with placebo. SCORE, as noted above, does not include CRP measurements. In their decision, the European Medicines Agency compromised between scientific evidence and economics, and their extension for rosuvastatin use in high-risk patients was admittedly arbitrary.

A recent reanalysis ${ }^{535}$ of cost-effectiveness demonstrated that rosuvastatin in JUPITER-eligible patients had an incremental cost-effectiveness of $\$ 25,198$ per quality-adjusted life year (QALY) gained compared to customary care. When applied only to patients with an FRS $\geq 10 \%$, the incremental cost-effectiveness became \$14,205 per QALY. Unknowns with respect to long-term effects cloud the issue, since sustained effects are assumed, but data is lacking. ${ }^{536}$ All proposals to improve risk refinement and lower risk burden, even nonselective administration of generic statins, will incurr expense.

The continuing debate about the use of statins in primary prevention, while quite apart from the JUPITER trial, has been connected to JUPITER for unclear reasons. ${ }^{15}$ Whenever use of statins in primary prevention is discussed, the cholesterol hypothesis, saturated fat-cholesterol link, JUPITER, and side effects of statins are also commonly argued de novo. Often in blogs and nonacademic publications the information presented is not evidence-based, but internet-based. Some authors have asserted that three-fourths of patients who take statin drugs for primary prevention - a significant number of all who use statins - do not benefit. ${ }^{292,537}$ Several guidelines from the American College of Cardiology, American Heart Association, and European Society of Cardiology disagree with this view. It has also been said that the JUPITER study masked or caused a loss of appreciation for primordial prevention ${ }^{23,24}$ but the debate concerning statin effectiveness in primary prevention predated the publication of JUPITER and is ongoing. ${ }^{36,298}$ No responsible cardiologist questions the value of primordial prevention before pharmacologic therapy. Individuals following a Mediterranean or Paleolithic diet who achieve ideal cardiovascular prevention would not need any therapy. ${ }^{538}$ The reality is that adherence to the Mediterranean diet, or any other lifestyle leading to ideal cardiovascular health, has fallen markedly even in areas of traditional origin - the Greek Islands - in favor of Western fare, accompanied by a corresponding increase in risk for CHD. Reversing this deterioration is the unmet challenge. The continuing fall in ideal cardiovascular health associated with poor lifestyle choices and the dual epidemics of obesity and diabetes are unrelated to the availability of statins or any particular application.

When lifestyle modification fails in individuals who are JUPITER-eligible, with "normal" LDL-C and high CRP levels, clinicians have an additional choice of using rosuvastatin to improve outcomes. This approach is simple, noninvasive, easily repeated, and does not involve radiation. CRP has been found useful in reclassifying risk in several series including the Framingham Heart Study, the Women's Health Study, the Physicians' Health Study, the Uppsala Longitudinal Study of Adult Men, the MONICA-Augsberg cohort, the EPIC Norfolk study, the Atherosclerosis Risk in Communities study, and the Heart and Soul cohort. The 2010 ACCF/AHA Guideline for Assessment of Cardiovascular Risk in Asymptomatic Adults, ${ }^{32}$ concluded that measuring CRP levels in JUPITER-eligible patients can be useful in the selection of patients for statin therapy, ie, that beginning statins in this population is reasonable, with recommendation Class IIa, Level of Evidence (LOE) of B.

\section{Increase utilization of imaging: CIMT and CAC}

\section{Carotid intima-media thickness (CIMT)}

CIMT is a useful, noninvasive, inexpensive, reproducible but operator-dependent, predictive, radiation-free, office-based technique. ${ }^{539-542}$ An increase in CIMT predicts the risk of cardiovascular events, but associations with cerebral arteriial events are stronger than with coronary events, relating more 
to variability and differences in atherosclerosis between the two arterial beds than to limitations in CIMT measurements. CIMT also provides information about noncalcified plaque. ${ }^{543}$ CIMT may detect significant atherosclerosis in patients with a zero calcium score, more likely to be young and/or female. Of 89 patients with a CAC of 0 , mean age 48 years, CIMT found evidence of carotid atherosclerosis in 42 (47\%; 95\% CI: $37 \%-58 \%) .{ }^{544}$ CIMT may be more sensitive than $\mathrm{CAC}$ in detecting subclinical atherosclerosis in a younger population, when treatment would produce greater benefits over time. It is believed that since calcification occurs later in the natural history of the disease, earlier stages of atherosclerosis - vulnerable plaque and noncalcified "inflammatory" lesions - may be found by CIMT. Therefore, CIMT may also find particular application in young healthy individuals, as well as in women and black patients. ${ }^{545}$ Once detected, however, coronary calcium is a much more powerful predictor of future coronary events than CIMT. ${ }^{219}$

CIMT has also been used effectively to monitor disease progression in individual patients, and in studies comparing properties of subgroups within a given diagnosis. In diabetes, for instance, CIMT has contributed to the understanding of vascular disease in people with normal glucose tolerance, impaired tolerance, overt diabetes, in those with hypertension, microvascular and other complications, and after treatment with hypoglycemic, antihypertensive, lipid-lowering and anti-platelet agents. ${ }^{546}$ As mentioned above, CIMT may be more appropriate as a surrogate in some populations and less adequate in others. ${ }^{547}$ Since the intima may thicken, and the media may become hypertrophic with age, not all elevations in CIMT in the elderly are due to atheroma.

Despite widespread use of CIMT serially to assess effects of therapeutic agents, a recent meta-analysis suggests limited usefulness for this purpose. Costanzo et $\mathrm{al}^{548}$ undertook a weighted random-effects meta-regression analysis to test mean and maximum CIMT changes and hard outcomes. They included 41 trials totaling 18,307 participants. Despite significant reductions in CHD and CVD events, as well as in all-cause mortality induced by various interventions, they found no significant relationship between CIMT regression and events in any of these categories. The surprising conclusion was that neither regression nor progression of CIMT changes correlated with, or predicted, changes in major cardiovascular events associated with various drug treatments in patients at intermediate to high cardiovascular risk. These findings, which disagree with the bulk of prior experience, may be due to limitations of the meta-regression analytic method and the limited length of follow-up in the
Table 8 Characteristics of CIMT and CAC by CT

\begin{tabular}{|c|c|c|}
\hline Quality of interest & $\begin{array}{l}\text { CIMT } \\
\text { (Ultrasound) }\end{array}$ & $\begin{array}{l}\text { CAC scoring } \\
\text { (CT) }\end{array}$ \\
\hline Area & Intima-media & Calcified lesions \\
\hline Radiation & None & $\begin{array}{l}0.7 \mathrm{mSv} \text { for EBT } \\
\text { and } 3-5 \mathrm{mSv} \text { for } \\
\text { helical CAC } \\
\text { (may vary } \\
10 \text {-fold up to } \\
10.5 \mathrm{mSv} \text { ) }\end{array}$ \\
\hline Sensitivity & 50 to 70 & 85 \\
\hline Specificity & 60 to 80 & 75 \\
\hline $\begin{array}{l}\text { Hazard ratio for incident } \\
\text { CVD event per SD } \\
\text { increment change title }\end{array}$ & 1.3 & 2.1 \\
\hline Reclassification improvement & Unknown & Yes \\
\hline Discrimination improvement & Uncertain & Yes \\
\hline Operation simplicity & ++ & +++ \\
\hline Operation reproducibility & User dependent & Automated \\
\hline Approximate cost & US $\$ 200$ & US $\$ 300-\$ 600$ \\
\hline Capitalization and access & $\begin{array}{l}\text { Low-within office } \\
\text { and research access }\end{array}$ & $\begin{array}{l}\text { High-larger } \\
\text { facility access }\end{array}$ \\
\hline
\end{tabular}

Abbreviations: CAC, coronary artery calcium; CT, computed tomography; CIMT, carotid intima-media thickness; EBT, electron beam tomography.

component studies. Replication of the data and explanation of inconsistencies are required.

Nonetheless, CIMT provides an excellent risk prediction tool which may be repeated easily. In the past 10 years, seven guidelines or consensus statements have recommended using CIMT or carotid plaque detection to predict risk. ${ }^{549}$ The USPSTF was not one of them. ${ }^{550}$ Among several potential applications of CIMT, appropriate use criteria rated seven as appropriate, 16 as uncertain, and 10 as inappropriate. ${ }^{364}$

The 2010 ACCF/AHA Guidelines for Assessment of Cardiovascular Risk in Asymptomatic Adults ${ }^{32}$ have assigned a Class IIa, LOE B recommendation for CIMT for risk assessment in asymptomatic individuals in the FRS intermediate risk category. Additional mention was made that recommendations for equipment, technical approach, operator training and experience for performance of the test must be followed. Some characteristics of CIMT and CAC are compared in Table 8.

\section{Coronary Artery Calcium scoring (CAC)}

Together with traditional risk factors, CAC also increases discrimination between patients who will or will not have future events, and improves prediction. ${ }^{551,552} \mathrm{CAC}$ is noninvasive, sensitive, automated, involves radiation exposure (0.7-1.8 $\mathrm{mSv}$ depending upon method), and is somewhat more expensive per test than CIMT, requiring greater operating costs and a much larger capital outlay. A great deal of data and a number of reviews have recently been published concerning 
the potential of CAC. ${ }^{193-195,553-562}$ Special note should be made of the Society for Heart Attack Prevention and Eradication (SHAPE) guideline, ${ }^{560}$ advocating early use of CAC in primary prevention patients ${ }^{563,564}$ with updated commentary. ${ }^{553}$

Nasir et al, ${ }^{565}$ reporting on of 1,611 asymptomatic individuals ( $67 \%$ men, mean age: $53 \pm 10$ years) who had CAC scores performed using single electron beam tomography, found that $59 \%$ of those with a CAC score $>400$ and $73 \%$ with a score $>75$ th percentile would not have been eligible for statins using NCEP ATP III criteria. CAC scores were able to reclassify $55 \%$ of patients classified as FRS-low risk to an intermediate risk category, and $45 \%$ of those with intermediate risk to high risk. In terms of biological age, linear prediction models showed that a CAC score $<10$ led to a reduction in observed age of 10 years in asymptomatic individuals over age 70, compared to those with a CAC score $>400$, which added up to 30 years of biological age to younger patients. ${ }^{566}$ Hence, a CAC of 0 has been called a "priceless" possession. ${ }^{567}$

A patient with some calcium has a relative risk $\geq 2$ compared with a CAC of zero, and in those with a CAC $>100$, the relative risk is $>4$. The greatest value of CAC scoring is in patients classified in the FRS intermediate risk group. The reclassification rate is $54 \%$, with $16 \%$ moving into the high risk category. In the Rotterdam study, ${ }^{556}$ the relative risk of a CHD event between the highest $11 \%$ and the lowest $50 \%$ of the calcification score distribution was 8.3. For individuals with a CAC of zero, the practitioner may be more inclined to avoid statins and aspirin, given their finite complication rates. A CAC over 100 might suggest aggressive LDL-C lowering with continuation of aspirin. For very high CAC values, over the 75 th percentile for age and gender, vigorous medical therapy is indicated with further work-up according to guidelines.

The MESA study ${ }^{219}$ found the association between incident cardiovascular events stronger with $\mathrm{CAC}$ than with CIMT (hazard ratio for incident event per SD increment 2.1 and 1.3 respectively), and an area under the receiver operating characteristic curve of 0.81 and 0.78 . While a popular subject for debate has been the relative advantages of CAC and CIMT for risk stratification in primary prevention, ${ }^{568}$ these techniques, as well as CRP measurement, are complementary clinical tools, rather than competitors.

For detection of plaque regression, CAC may not be reliable. Some reservations have also been voiced about the clinical usefulness and cumulative radiation exposure in heart patients. ${ }^{199,224,568-579,633}$ While the radiation exposure per procedure is now lower with new technology, exposure is uneven. Cumulative radiation exposure in adults, even in children, is rising, since imaging for all purposes is becoming common. ${ }^{58-582}$ In view of the long delay between exposure to ionizing radiation and development of cancer, as well as its certainty, researchers have suggested that enthusiasm for cardiac imaging should be tempered. ${ }^{579}$

Another potential distraction is the finding of "incidentalomas" on imaging - about $20 \%-53 \%$ of electron-beam CT and $15 \%-67 \%$ of multidetector row CT report extracardiac lesions. About 5\%-11\% are significant, with 4\%-25\% of them potentially significant. ${ }^{583}$ Additional tests, expense, inconvenience, professional time, and patient anxiety may follow. The US Preventive Services Task Force (USPSTF) disfavors CAC because it leads to additional testing and may funnel asymptomatic individuals to catheterization laboratories. In contrast, advocates cite some tests and revascularizations that may also be avoided. When patients are found to have a zero calcium score, less aggressive LDL-C targets translate into less expensive protocols with generic statins, but the quantitative significance of this remains uncertain.

An intriguing study from Johns Hopkins School of Medicine reported on the use of CAC in 950 healthy male and female participants with LDL-C $<130 \mathrm{mg} / \mathrm{mL}$ and $\mathrm{CRP} \geq 2 \mathrm{mg} / \mathrm{L}$ from the MESA population over a 6-year period. ${ }^{221}$ About $47 \%$ had a calcium score of $0,28 \%$ had a calcium score of 1 to 100 , and 25\% were in a high-risk group with a calcium score over 100 . About $75 \%$ of the deaths related to CVD events occurred in the highest-risk group. According to these data, in individuals with a calcium score of 0 , the CHD event rate was 0.8 per 1000 patient-years, the number needed to treat (NNT) to prevent one CHD event was 549, and for a CVD event, NNT was 124. In the group with a score between 1 and 100, the event rate was 4.8 per 1000 patient-years. In the group with CAC $>100$, the CHD event rate was 20.2 per 1000 patient-years, and the NNT to prevent 1 CHD event was 24, and for 1 CVD event, 19. The hazard ratio for a CHD event in the highest-risk group of 24.8 (95\% CI 2.5-14.6) is indeed convincing. Placing this information in perspective will require further randomized trials. These data clearly show that a significant amount of cardiovascular risk and incipient pathology exists within the asymptomatic, nondyslipidemic population who do not presently qualify for further medical attention. More patients are now presenting with LDL-C values that are not especially elevated, which may elicit undertreatment with statins (see above discussion).

The use of CAC as a noninvasive test for risk stratification of emergency department (ED) patients with chest pain in order to decide whether to proceed with coronary angiography or discharge is a different, although similarly controversial, 
issue than stratification of asymptomatic individuals classified as intermediate risk using FRS. Sarwar and colleagues ${ }^{584}$ reported data from 18 studies that revealed any $\mathrm{CAC}$ had a pooled sensitivity and negative predictive value of $98 \%$ and 93\%, respectively, for finding significant CHD on invasive coronary angiography. Even so, Garcia and Fuster ${ }^{585}$ noted that from those same data, while the incidence of obstructive $\mathrm{CHD}$ in chest pain patients with zero CAC scores is small, at $7.2 \%$ it is not negligible. Candemartiri et $\mathrm{al}^{586}$ found that CAC scoring was inadequate when compared to computed tomography coronary angiography (CTCA) in excluding CHD in asymptomatic, high-risk patients. Gottlieb et $\mathrm{al}^{587}$ reported that the absence of coronary calcification (CTCA) had a positive predictive value of $81 \%$, and a lower negative predictive value of CAC of $68 \%$, and therefore a zero CAC score does not reliably rule out significant $\mathrm{CHD}$ in patients being referred for coronary arteriography. Of those, about $20 \%$ had a high pretest probability of CHD, 75\% had an intermediate, and 5\% had a low probability of CHD. In an accompanying editorial, Redberg ${ }^{588}$ commented the practice should be discouraged, largely due to the failure to predict $19 \%$ of patients with $\mathrm{CHD}$ as well as a finite radiation risk. While the radiation for CAC is minimal, with a median value of $\approx 3 \mathrm{mSv}$, roughly equal to that of a mammogram or 100 chest $x$-rays, the variation is considerable $(2-7 \mathrm{mSv}),{ }^{224}$ and multiple scans in the same patient are becoming more common. Generally, patients' perceptions of cumulative $\mathrm{CT}$ radiation risk are inaccurate. ${ }^{589}$ Three years ago, the number of future malignancies from CT scans done in 2007 was estimated at $\approx 29,000$, corresponding to some 15,000 deaths. ${ }^{590}$ In contrast, supporters argue that CAC scores are of immense help in evaluating chest pain in the ED and elsewhere. ${ }^{195,233,567,592-594}$

A summary of the utility of CAC in risk evaluation ${ }^{594}$ concludes, as do guideline writers, that the absence of detectable coronary calcium is associated with a favorable prognosis, but is imperfect and carries a limited (92\%) warranty of about $4.1 \pm 0.9$ years. ${ }^{223}$ While CAC measurement does refine risk stratification above that provided by the FRS in asymptomatic, intermediate-risk patients, whether doing CAC scoring earlier in more patients will result in improved long-term clinical outcomes has yet to be decided, and doing so will be difficult. Restricting use based upon lack of such data may be too stringent a requirement. A larger question is whether CAC used to assess higher risk is superior to a population based strategy. In response to correspondence regarding the role of CAC scoring, Redberg ${ }^{595}$ opined that, despite two decades of study, data showing patient benefits from information derived from the CAC are still lacking, and that for this reason, the
USPSTF concluded the evidence regarding CAC score is insufficient to assess the benefit to risk ratio. ${ }^{550}$

Baseline measurements of CAC have received a Class IIa, LOE B recommendation (reasonable) for those at intermediate (10\%-20\% 10-year) risk or Class IIb for individuals at low to intermediate risk by the $2010 \mathrm{ACCF} / \mathrm{AHA}$ Guidelines for Assessment of Cardiovascular Risk in Asymptomatic Adults, and for those at low to intermediate (7\%-10\% 10-year) risk, a Class IIb, LOE B recommendation. ${ }^{32}$ No recommendations have been made for CAC progression for assessing treatment. CAC progression may provide even more valuable information, but also requires sequential $\mathrm{CT}$ scans with additional radiation exposure, together with other limitations. ${ }^{596}$

\section{Allocate statins to low- and intermediate- risk individuals using specified risk thresholds, rather than ATP III or JUPITER criteria}

There is some evidence that extending statin therapy to Framingham low- and intermediate-risk individuals may be cost-effective at all levels of LDL-C. ${ }^{316,597,598}$ Reservations about such a policy arise from potential side effects and expense. As was done in JUPITER, a CRP $\geq 2.0 \mathrm{mg} / \mathrm{L}$ can be used to identify those persons with higher risk in a population without ATP III-defined dyslipidemia who might benefit from statin therapy. A different approach to efficient lowering of cardiovascular risk was suggested by Lee et al. ${ }^{302}$ These investigators examined the cost effectiveness of strategies following three hypothetical cohorts of individuals starting at age 40 with normal lipid levels and no diagnosed coronary artery disease, peripheral arterial disease, or diabetes. Their Markov decision analytic model compared three situations using: a) ATP III guidelines, a current popular strategy; b) CRP screening in JUPITER-eligible patients, followed by statin treatment only for those with CRP elevations, as suggested by the JUPITER study; and c) a strategy of starting statin therapy at specified predicted risk thresholds without first performing any CRP testing. Assuming that the relative risk reduction in events by statins is uniform whatever the baseline risk might be (statins are equally effective regardless of CRP status, providing benefits in low-risk individuals with normal LDL-C and CRP levels) the most cost-effective strategy was c). Thus, treating individuals at significantly lower risk than those currently being treated without using CRP screening was favored by these authors. However, if a normal CRP level meant that little or no benefit would result from statins, than strategy 
b), treating the dual targets of LDL-C and CRP, would be the most cost-effective strategy. Notably, however, this analysis did in fact show that CRP testing is superior to current ATP III guidelines for individuals at "intermediate" FRS risk.

Other assumptions in this study were that statins remained inexpensive (generic simvastatin was used for their calculations), $17.5 \%$ of participants would discontinue statins within 6 months due to intolerance, rhabdomyolysis and renal failure would occur in 5-30 patients per 1 million treated with statins, and there were no long-term adverse effects of statins. If patient adherence to statin drugs was in fact poorer for any reason, ${ }^{90}$ the calculations for costeffectiveness would become invalid. In fact, the lead author of the paper remarked in a subsequent interview that "it doesn't take much to make statins not cost-effective for such large-scale use".

In their equal-effects scenario, it was assumed relative risk reductions from statins were not a function of FRS or CRP levels, and that statin therapy lowered the risk of MI by a factor of 0.77 , and the risk of stroke by a factor of 0.83 . In the differential scenario, it was assumed that individuals with high CRP values modified statin effectiveness, with relative risks of 0.46 for $\mathrm{MI}$ and 0.52 for stroke (data from JUPITER). An interactive presentation of the model with variable risk factors is accessible at http://med.stanford.edu/ hsr/crp-screening.

The assumption of uniformity of effectiveness of all statins for all individuals, across ethnic and other groups, regardless of LDL-C, HDL-C, lipid subfractions, CRP and other biomarkers should be noted. If a normal CRP could exclude effectiveness of statins, then CRP-guided therapy would be best. According to the authors, because JUPITER did not have a normal LDL-C/normal CRP group for comparison, it remains unknown whether CRP elevations merely increases risk, or a normal CRP indicates ineffectiveness of statins in such a low/normal-CRP cohort. However, even though JUPITER did not have such an arm, data from other sources suggest that CRP does have discriminatory capability. In the AFCAPS/TexCAPS study, the relative risk reductions associated with the use of statins in patients with high CRP values was $42 \%$, higher but not significant compared to the lower risk reductions in patients with normal CRP levels. ${ }^{599}$ A subanalysis of JUPITER did demonstrate a relationship between outcomes in rosuvastatin-treated individuals and CRP levels. ${ }^{279}$

There was no consideration for any differential in efficacy, side effects, or potential interactions between rosuvastatin and simvastatin, but these potential differences may become important clinically. Simvastatin, as a highly lipophilic statin, is associated with a higher incidence of both myopathy and other adverse reactions than rosuvastatin, which is hydrophilic. ${ }^{15}$ Further, since simvastatin is metabolized through the CYP3A4 enzyme, the probability of interactions with coadministered CYP3A4 substrates, inhibitors and inducers is much greater than with rosuvastatin, and package inserts by manufacturers reflect these data. For instance, simvastatin dosage is restricted when coadministered with with amiodarone and verapamil due to such interactions. Since polypharmacy is increasingly common, this may be a consideration in such a public health proposal.

In conclusion, if all the assumptions were true, then giving simvastatin $80 \mathrm{mg}$ daily to all men who have no risk factors, without CRP testing, beginning at age 55, would be cost-effective, defined as less than $\$ 50,000$ per qualityadjusted life-year (QALY). For men with one risk factor, eg, hypertension, simvastatin would be cost-effective beginning at age 50, and with 2 risk factors, at age 40 . Data generated by this model provides some insight into possibilities in lieu of a large, costly, long-term clinical trial. ${ }^{600}$

\section{Allocate statins using a risk-based, "tailored" strategy, rather than a standard or intensive ATP III treat-to-target approach}

Five years ago Hayward et $\mathrm{al}^{601}$ questioned whether treating to different LDL-C targets was actually evidence-based. These investigators reviewed controlled trials, cohort studies, and case-control studies that examined the relationship between lowering cholesterol levels and cardiovascular outcomes in patients with LDL-C $<3.36 \mathrm{mmol} / \mathrm{L}(<130 \mathrm{mg} / \mathrm{dL})$. There was no support for the premise that the response of LDL-C to statins predicted the degree of cardiovascular risk reduction. It was concluded that patients with high risk should be treated with statins regardless of their initial LDL-C level.

Drawing from NHANES data (1977-1994), using the FRS, and employing a simulated model of population-level lifetime effects of 5 years of treatment with statin drugs, Hayward and coworkers ${ }^{598}$ compared two treatment strategies. They chose either a conventional approach, using escalating treat-to-target NCEP-ATP III guidelines (in standard and more intensive options), or a risk-based approach (patients with 5\%-15\% CHD risk receiving $40 \mathrm{mg}$ simvastatin daily, and those with $>15 \%$ CHD risk receiving $40 \mathrm{mg}$ atorvastatin daily). Participants were 30-70 years of age without a history of myocardial infarction. The investigators found that $\approx 70 \%$ 
of patients would be treated similarly using the two strategies, $14 \%$ would be treated more aggressively using the risk-based approach, and 17\% would receive more aggressive therapy following the treat-to-target protocol. Intensive treat-to-target treatment resulted in 15 million more people being treated and saved 570,000 more quality-adjusted life-years over the 5-year period. The tailored risk-based strategy resulted in just as many people being treated as intensive treat-to-target therapy, saved 520,000 more quality-adjusted life-years, but did not require as many patients to take high potency statins. More CHD events were prevented with the risk-based approach compared with the treat-to-target strategy, with rates of 62 and 15 per 1000 treated patients respectively. The treat-to-target strategy resulted in treatment of more patients with higher LDL-C levels, but with lower CHD risk, whereas the risk-based approach caused treatment of a greater number of patients with elevated CHD risk, but with lower LDL-C levels.

Using the risk-based approach incorporates the log-linear association between lowering LDL-C and CHD risk, which is maintained at lower values of LDL-C. The strategy also addresses the population-wide underutilization of statins in general. ${ }^{602}$ In addition, the risk-based approach does not require monitoring of $\mathrm{LDL}-\mathrm{C}$, fewer physician encounters would be involved, and provides a simple prevention plan, eliminating guideline and treatment confusion among clinicians. Despite these advantages, and even if validated using a randomized trial, physician and patient acceptance would be difficult.

In countries where individualized care is not yet traditional and budgetary constraints are primary, a tailored population-based approach to achieve the most risk reduction will be well received. At each risk level, for cost-effectiveness, two people taking low-dose simvastatin provides better returns than one taking atorvastatin or rosuvastatin. Variations on this tailored treatment theme can be used to manage resources in optimizing population benefits with different statin schedules. ${ }^{603,604}$ In actual practice with higher risk patients, the reduction in events may still be too low. Many cardiologists believe that limiting therapy to statins alone, the only class of agents for which significant evidence exists for hard-outcome efficacy, ${ }^{605}$ will not result in lowering events by $50 \%$, particularly using simvastatin.

Limitations in the use of surrogate biomarkers rather than patient outcomes in the treatment of dyslipidemia were discussed by Krumholz and Hayward. ${ }^{606}$ Treating risk factors is a time-honored technique that has provided mechanistic understanding of the pathogenesis of atherothrombotic disease, and is endorsed by the FDA. From a systems biology point of view, however, these authors observe that understanding mechanisms does not necessarily lead to improved patient outcomes. They note that a) statins lower risk of MACE, revascularization and stroke, ${ }^{233,291}$ quite apart from the baseline lipid level; b) there is little or no evidence that combination therapy to achieve lipid targets actually improves patient outcomes; and c) the strategy used is more important than the change in cholesterol. To be sure, examples include the failures when torcetrapib, estrogen, clofibrate, and dextrothyroxine were used to lower cholesterol levels without reducing risk, and the absence of adequate evidence to support improvement in MACE associated with use of ezetimibe.

In the UK, because there are no data directly comparing high and low intensity statin therapy in primary prevention, treatment is conservative, using a "systematic rather than opportunistic" risk assessment, and simvastatin $40 \mathrm{mg}$ is prescribed for individuals with a 10 -year risk of $10 \%-20 \%$. Titration to LDL-C targets and lipid and other monitoring is deemed unnecessary, and offering additional anti-lipid therapy is not routinely advised. ${ }^{283,285,301,607}$

With all proposals to lower cardiovascular risk, poor adherence is frequently the elephant in the room. As Rose ${ }^{47}$ pointed out, a disadvantage of population- vs individual-based prevention is the small perceived benefit to the individual. When patients insist upon seeing objective improvement in their own risk factors as a requirement to continue taking simvastatin, adherence tends to wane.

\section{Redefine the "normal" LDL-C level and eliminate multiple LDL-C goals}

Defining normal LDL-C values based upon a Gaussian distribution in "asymptomatic" individuals repeats intrinsic errors because symptomatology is a poor index of either the activity or stage of atherosclerotic disease. The asymptomatic population is heterogeneous as far as cardiovascular risk, current pathology, and future events, and may not be "normal". The mean untreated adult LDL cholesterol value is $\approx 130 \mathrm{mg} / \mathrm{dL}$ $(\approx 3.4 \mathrm{mmol} / \mathrm{L})$ in the USA, but lowering LDL-C levels to $100 \mathrm{mg} / \mathrm{dL}-130 \mathrm{mg} / \mathrm{dL}$ only prevents $25 \%-35 \%$ of events, with symptoms frequently dissociated from pathology. Evidence from contemporary hunter-gatherer societies and from wild primates suggests that humans are genetically better matched with "physiological" levels of LDL-C that may be on the order of $35 \mathrm{mg} / \mathrm{dL}-50 \mathrm{mg} / \mathrm{dL} .{ }^{608}$ Indeed, extrapolation of data from several meta-analyses indicate that in primary prevention, cardiovascular events would nearly be 
eliminated at LDL-C $<60 \mathrm{mg} / \mathrm{dL}$. In secondary prevention the corresponding threshold would be LDL-C $<30 \mathrm{mg} / \mathrm{Dl}{ }^{607}$ Humans are distinguished by uniquely elevated LDL-C values compared with wild mammalian herbivores, carnivores, and omnivores. Convincing data indicate that the "Western" atherogenic diet is primarily responsible. ${ }^{610,538}$

Only a short time ago, the overriding concerns were basically: could such low LDL-C values be achieved; would it be safe to do so; and would sufficient clinical benefits be realized? The current tentative answers appear to be: yes; yes; and probably yes. Noting that answers to the last question must rest upon logical inference rather than hard evidence, Forrester ${ }^{255}$ cites the $>60 \%$ decrease in cardiac events associated with a lifetime of LDL-C lowering in PCSK-9 hypofunction mutations to argue for prolonged statin therapy, and challenges the currently accepted "normals" for LDL-C levels. Further, a single "physiological" or putative normal LDL-C level - 50-70 mg/dL - could replace multiple targets presently assigned by global risk. Such an approach is supported by pathologic, epidemiologic and clinical trial data. ${ }^{233}$

The evidence that at very low levels of LDL-C cardiovascular events may approximate zero comes from several venues. First, comparative physiology of mammals and early human life indicate a genetic set-point for LDL-C that is less than half of the average untreated value in the Western world, $\approx 3.4 \mathrm{mmol} / \mathrm{L}(130 \mathrm{mg} / \mathrm{dL})$. Feeding studies in many species of mammals vs those in the wild demonstrate a dose-related association of the Western diet with atherosclerosis. Near absence of atherosclerosis is associated with longevity and low LDL-C levels in contemporary huntergatherer societies. ${ }^{610,611}$ When diets of such individuals are westernized, a rise in LDL-C and atherosclerosis progress hand-in-hand. ${ }^{612}$ Extrapolation of event rates at progressively reduced LDL-C levels in primary and secondary prevention trials using statins implies that at very low levels, as mentioned above, events would virtually be eliminated. ${ }^{608,609}$

In a meta-analysis conducted by the Cholesterol Treatment Trialists' Collaboration, ${ }^{233}$ a total of 170,000 high-risk participants in 26 randomized trials who began with an LDL-C of $1.8 \mathrm{mmol} / \mathrm{L}(70 \mathrm{mg} / \mathrm{dL})$, were treated down to $\approx 1.3 \mathrm{mmol} / \mathrm{L}$ $(50 \mathrm{mg} / \mathrm{dL})$. With each $1 \mathrm{mmol} / \mathrm{L}$ reduction, the number of occlusive vascular events fell by about $20 \%$, regardless of baseline LDL-C. However, despite the suggestion that stringent reduction of LDL-C could lower risk by about $40 \%-50 \%$, reducing events across the board by $50 \%$ in the general population, especially with simvastatin, has yet to be demonstrated. In a large study of asymptomatic primary care patients with LDL-C $<130 \mathrm{mg} / \mathrm{dL}$ and CRP $\geq 2 \mathrm{mg} / \mathrm{L}$, those who who attained LDL-C $<50 \mathrm{mg} / \mathrm{dL}$ when treated with rosuvastatin $20 \mathrm{mg}$ showed a striking $65 \%$ fall in the risk of cardiovascular events and a $46 \%$ reduction in total mortality. ${ }^{344}$ Benefits were not associated with either the baseline LDL-C level or with a significant increase in adverse events. These data are consistent with the view that very low levels of LDL-C $<70 \mathrm{mg} / \mathrm{dL}$ may be achieved safely and produce greater improvements in outcomes than nonaggressive therapy. When indicated, lower appears to be better.

Setting a goal equal to the "physiologic" LDL-C in almost everyone has an immense advantage of simplicity. The ease with which this policy could be applied might raise adherence by practitioners and patients alike. However, this bold proposal is also accompanied by some uncertainties and caveats. Drawbacks related to toxicity, cost, and differences in potency may preclude achieving putatively physiological LDL-C levels in at least $25 \%$ of patients. Risk also varies according to variables other than LDL-C levels, including HDL-C, other triglyceride rich fractions, non-lipid risk factors, inflammation, enzyme activities, etc. Many individuals may resist preventive treatment, because they do not feel ill, with no discernible immediate physical benefit, added inconvenience and cost, and fear of side effects, which are widely disseminated on the internet. These factors may make adherence poorer than current levels, not better. The extent of adherence is probably of greater importance than the particular approach used.

It should be emphasized the notion that achieving an LDL-C of $50 \mathrm{mg} / \mathrm{dL}$ would lower cardiac events to negligible levels is theoretical, not proven. For example, many doubt that cardiovascular events would be eliminated in diabetics if LDL-C levels of $50 \mathrm{mg} / \mathrm{dL}$ - values observed in rats, cattle, and deer - were achieved. Such a prediction assumes that nearly $100 \%$ of CHD events may be explained by LDL-C elevation above that putative "physiological" value. In addition, present-day hunter-gatherer individuals experience a different life than those in developed civilizations, with a sizeable difference in exercise level, stress and pollutant exposure. Hence, while more intensive reduction in LDL-C will produce significant improvements in reducing cardiovascular events, only a partial, rather than total, amelioration will likely result from this proposal.

\section{Follow treat-to-target lowering of LDL-C, but raise HDL-C}

Keeping in mind that just $\approx 30 \%$ of cardiovascular events are prevented with statins, and even when maximally tolerated doses are used, only a further $16 \%$ can be suppressed, Drexel 
et $\mathrm{a}^{613}$ sought to identify the factors that were responsible for the residual risk in non-diabetic and diabetic patients. In a small study, vascular events were recorded over 5.6 years in 491 consecutive statin-treated patients with angiographicallyproven stable CHD, amounting to 2750 patient-years. High values of triglycerides, small dense LDL, and low values of HDL-C and apoA-I predicted vascular events, but not LDL-C or apoB levels. In a small observational study, such results were only suggestive, but highlighted the potential importance of low HDL-C as a predictor of cardiovascular risk, especially in statin-treated patients. In a larger $(n=2910)$, community-based sample in the Framingham Heart Study, additional CHD risk associated with high TG or low HDL-C levels was only found in patients with insulin resistance. ${ }^{630}$

There has been considerable progress in understanding HDL metabolism, and interest in raising HDL-C levels as a means of reducing risk is keen. Larger ongoing clinical trials that target specific pathways in HDL metabolism may provide sufficiently robust data to support new treatment options. The present investigative focus includes increasing HDL-mediated reverse cholesterol transport, raising the proportion of more effective HDL subfractions, or producing functioning human apoA-I or surrogate molecules. Generally, there is only modest evidence showing that raising HDL-C, in addition to what is achieved by lifestyle modification alone, will improve outcomes. ${ }^{358}$ Both the European ${ }^{31}$ and Canadian ${ }^{190}$ guidelines for dyslipidemia management emphasize that trial data do not show pharmacological treatment of HDL-C will lower cardiovascular risk. Indeed, recent reports using fibrates and niacin to lower risk have not changed this view.

\section{Use a modified traditional approach which preserves LDL-C targets}

Nambi and Ballantyne ${ }^{227}$ proposed a formal protocol using the FRS, lifetime risk, and CRP, CIMT, CAC or genetic risk markers for further progressive refinement of stratification. After initially estimating FRS, if risk was $>20 \%$, the LDL-C goal would be $70 \mathrm{mg} / \mathrm{dL}$. If FRS was low, or $0 \%-10 \%$, lifetime risk would then be determined. For individuals with both a low 10-year and low lifetime risk, the prevailing NCEP-ATP protocol would apply. For those with a low 10-year risk, but a high lifetime risk, further stratification using CRP, CIMT, CAC score or genetic risk markers would be undertaken.

Finally, for those with an FRS of 10\%-20\% at intermediate risk, the current ATP III guidelines advise an LDL-C goal of $<130 \mathrm{mg} / \mathrm{dL}$ and an optional goal of $<100 \mathrm{mg} / \mathrm{dL}$, which may change in ATP IV guidelines. These investigators also agree that the LDL-C value at birth, $\approx 50 \mathrm{mg} / \mathrm{dL}$, may be physiological, and cardiovascular benefits would be expected until those values are reached.

\section{Give statins to low- and intermediate-risk individuals within a polypill}

The polypill concept was proposed by Wald and Law ${ }^{614}$ to lower LDL-C, blood pressure, serum homocysteine, and inhibit platelets regardless of pretreatment levels in a large segment of the population (over age 55), produce few side effects, with minimal expense. As safety of treatment rises and expense falls, risk stratification is considered less valuable. One-third of people taking this pill from age 55 were expected to benefit, adding an average of 11 years to life, free from a CHD event or stroke. ${ }^{614}$

In the Indian Polycap Study (TIPS), a polycap containing low doses of thiazide $(12.5 \mathrm{mg})$, atenolol (50 mg), ramipril (5 mg), simvastatin (20 mg), and aspirin (100 mg) was studied in 2053 Indian subjects without cardiovascular disease, but with at least 1 risk factor ${ }^{613}$ Based upon the results, there was a potential for a $62 \%$ reduction in relative risk in CHD and a $48 \%$ lowering of relative risk for stroke. This was short of the $80 \%$ risk reduction originally envisioned. ${ }^{614,616}$ Not surprisingly, even though well-tolerated, acceptance and adherence was still a significant problem. A polypill feasibility study in Sri Lanka sponsored by $\mathrm{WHO}^{617}$ found high patient acceptability, which need not have correlation with future adherence or outcomes. Compared to other techniques, the use of a polypill does not depend heavily upon personal responsibility for lifestyle change, since instructions are simple. Unfortunately, adherence to poor diets and inactivity is greater than adherence to polypills, and the protection afforded by the polypill, although impressive and with many advantages, ${ }^{618-620}$ remains incomplete. Although it will go a long way in reducing cardiovascular risk, final long-term success in hard end points remains to be seen. The Use of a Multidrug Pill In Reducing cardiovascular Events (UMPIRE) study is beginning in the UK and in other venues, while other studies sponsored by WHO are in progress. In populated poor countries, the polypill may ultimately provide better protection than expensive, sophisticated care.

\section{Conclusion}

Alternatives for improving cardiovascular prevention based upon evolving concepts, new data, and revised goals have changed remarkably in recent years. In primary prevention, traditional risk factors used in combination to generate global scores do not predict risk well enough, nor do they discriminate sufficiently between those who will have cardiovascular events and those who will not. Chosing the best mix of approaches for 
cardiovascular prevention cannot presently be based upon hard end point data, but partially upon an evidence-based synthesis using inductive reasoning. Even though the original belief that prevention was cost-ineffective has now been disproven, ${ }^{621}$ at least for cardiovascular applications, there has been disappointing progress in effecting successful population-based primordial prevention. For truly effective improvements in cardiovascular risk, primordial prevention appears necessary as an adjunct to the high-risk strategy traditionally offered to individual patients. Wilkins and Lloyd-Jones ${ }^{526}$ explicitly declare that the present paradigm of identifying high-risk individuals alone will never succeed in lowering the risk burden, even without considering further progression of obesity and diabetes.

The magnitude of the problem - pervasive poor cardiovascular health and its importance - has not been fully appreciated. Psychosocial aspects of behavior in embracing and adhering to primordial, primary, and secondary prevention are receiving greater attention. ${ }^{85-87,622-626}$ According to one health belief model, negative health behavior is in part due to the widespread failure of people to accept disease preventives when disease is asymptomatic. ${ }^{627}$ Part of the complex belief system involved leads to unrealistic optimism of vulnerability. ${ }^{628}$ During assessment, the psychology and inaccuracy of patient perceptions of risk and the factors leading to physicians' underestimation of patients' risk are significant and incompletely understood. ${ }^{170-173,198}$ As far as the estimation of cardiovascular risk burden is concerned, the chasm between perception and reality persists for both physicians and patients in North America, UK, and the EU. Given the lack of success and resistance to primordial prevention, population-wide pharmacological reduction of risk, previously rejected because of expense and potential side effects, is being reevaluated as a cost-effective maneuver. If one restricts evidence-based cardiovascular risk reduction to statins, the question reduces to what segment of the population will be eligible for how much of what statin or polypill.

Recent evidence suggests that attention to pediatric patients, at a time when habits are formed, and monitoring of adolescents as well as young adults, must increase.

The 2010 ACCF/AHA Guideline for Assessment of Cardiovascular Risk in Asymptomatic Adults, ${ }^{32}$ a consensus of experts, reaffirmed a central role for global risk scoring in assessing risk in all adults. Similarly, a family history was recommended for all patients. While recognizing the large risk burden in asymptomatic adults, difficulty in identification of patients who ultimately suffer cardiovascular events, the significant number of patients who remain untreated and eventually succumb to CHD deaths, this guideline set forth evidence-based recommendations for the identification and stratification of patients at risk.

Use of the Reynolds Risk Score, particularly in women, deserves consideration. ${ }^{629}$ Lowering the threshold for risk level may bring more care to patients who will benefit. In the 2011 American Heart Association update to the guidelines for prevention of cardiovascular disease in women, ${ }^{629}$ "high risk" among women is now defined as a 10 -year CVD risk $\geq 10 \%$ rather than $\geq 20 \%$.

Of all biomarkers, CRP is the best studied circulating biomarker, and provides information about activation of upstream cytokines driving inflammation. High levels of CRP are associated with endothelial dysfunction and predict future cardiovascular events. Use of C-reactive protein is recommended or accepted in guidelines for specific patients with intermediate risk. ${ }^{15}$ Among imaging techniques, CIMT reflects intima-media thickening due to progression of atherosclerosis, refines risk assessment beyond global risk scores, improves predictability, and has been used successfully for serial re-evaluations. CAC documents the extent of calcification, a process which occurs later in the evolution of atherosclerosis. Patients with higher CAC scores generally have advanced, diffuse disease with accompanying noncalcified vulnerable lesions, accounting for its high prognostic value. Increasing appropriate use of CAC and CIMT, according to clinical circumstances, will refine risk evaluation, help guide treatment, and probably improve outcomes. Use of both CRP and CAC, since information provided involve orthogonal mechanisms, may offer unique advantages. ${ }^{631,632}$ Recent work suggests that elevations in CRP levels predict a higher burden of coronary plaque, particularly mixed calcified arterial plaque, in asymptomatic individuals. ${ }^{634}$ Rises in CRP concentrations appear to be associated with vulnerable plaque, drawing still more attention to the important role of inflammation in atherosclerosis. ${ }^{635}$

In patients with high risk, aggressive treatment to reduce risk factors should be instituted early and maintained for years. Since the incubation period and signs and symptoms of atherosclerosis span decades, randomized trials of a few years' duration provide little insight into outcomes of statin treatment over those 40-60 years. The most aggressive stance would include immediate treatment of patients with high- (FRS $\geq 20 \%$ or equivalent) or even intermediate-risk (FRS 10\%-20\%) with high potency statins. Rosuvastatin and high dose atorvastatin are drugs of choice, even if the baseline LDL-C is not elevated. Rosuvastatin produces the greatest reduction in LDL-C, LDL-P, and improvement in apoA-I/apoB with a favorable safety profile. Lowering current 
LDL-C goals will undoubtedly enhance risk control and reduce event rates. However, even if all such patients received statins, cardiovascular events would still continue. The search for additional methods to lower residual risk, including using non-HDL-C as a target, raising functional HDL-C levels, ${ }^{636}$ and mining LDL-C subfractions for clinically useful information, continues. The recent negative trials using torcetrapib, fibrates, and extended-release niacin are significant and collectively discouraging. This is particularly true concerning niacin in AIM-HIGH, in view of prior evidence supporting the use of this drug in a meta-analysis of 14 smaller trials ${ }^{637}$ and ARBITER 6-HALTS. Nevertheless, the data reinforce the theme of this paper: intensive, unrelenting, lifestyle improvement and aggressive statin therapy are two pillars of management in the prevention of cardiovascular disease.

The enormity of pervasive poor cardiovascular health and its importance have not been fully appreciated and valued by the public, the media, health policy authorities, or legislators. Barring major unpalatable political changes, current trends in cardiovascular risk will undoubtedly continue. As such, there will be an even greater demand for pharmacological and invasive therapies. Most likely, success will be achieved through a combination of ongoing improvements in adherence, guideline compliance, novel treatments, and the valuable addition of primordial prevention in the form of programs such as the American Heart Association's Life's Simple $7^{\mathrm{TM}}$. Cooperation is essential, and each one of us - citizens, patients, biochemists, physicians, researchers, administrators, and public officials - have an essential role in supporting this common goal. Obstacles will be many, and the road hard and long, but recent advances now offer us greater and unique opportunities to meet the imposing challenge.

\section{Acknowledgment}

The author wishes to thank Michelle Delaney for her astuteness, computer skills, untiring assistance, and valuable suggestions in the preparation of this manuscript.

\section{Disclosure}

The author reports no conflicts of interest in this work.

\section{References}

1. Lloyd-Jones D, Adams R, Carnethon M, et al. Heart disease and stroke statistics - 2009 update: a report from the American Heart Association Statistics Committee and Stroke Statistics Subcommittee. Circulation. 2009;119(3):480-486.

2. Lloyd-Jones DM, Hong Y, Labarthe D, et al. American Heart Association Strategic Planning Task Force and Statistics Committee. Defining and Setting National Goals for Cardiovascular Health Promotion and Disease Reduction: The American Heart Association's Strategic Impact Goal Through 2020 and Beyond. Circulation. 2010;121(4):586-613.
3. Kones R, Phillips JH. Prevention of Heart Cell Death. In: Yu PN, Goodwin JF, editors. Progress In Cardiology, Vol 4. Philadelphia, PA: Lea and Febiger; 1975:199-224.

4. Health, United States, 2009: with special feature on medical technology. Hyattsville, MD: National Center for Health Statistics, 2010. F38.

5. Institute of Medicine. "A population-based policy and systems change approach to prevent and control hypertension" 2010; Washington, DC: The National Academies Press. Brief report available at: http:// www.iom.edu/Reports/2010/A-Population-Based-Policy-and-SystemsChange-Approach-to-Prevent-and-Control-Hypertension/Report-BriefPrevent-and-Control-Hypertension.aspx. [22 Febraury 2010]. Accessed March 24, 2011.

6. Fuster V, Kelly BB, editors; for the Institute of Medicine (US) Committee on Preventing the Global Epidemic of Cardiovascular Disease: Meeting the challenges in developing countries, promoting cardiovascular health in the developing world: a critical challenge to achieve global health. Washington, DC: National Academies Press (US); 2010. The National Academies Collection: Reports funded by National Institutes of Health.

7. Heidenreich PA, Trogdon JG, Khavjou OA, et al. Forecasting the Future of Cardiovascular Disease in the United States: A Policy Statement From the American Heart Association. Circulation. 2011;123(8):933-944.

8. Capewell S, Ford ES, Croft JB, Critchley JA, Greenlund KJ, Labarthe DR. Cardiovascular risk factor trends and potential for reducing coronary heart disease mortality in the United States of America. Bull World Health Organ. 2010;88(2):120-130.

9. Stewart ST, Cutler DM, Rosen AB. Forecasting the effects of obesity and smoking on US life expectancy. $N$ Engl J Med. 2009;361(23): 2252-2260.

10. Crimmins EM, Preston SH, Cohen B, editors; Panel on Understanding Divergent Trends in Longevity in High-Income Countries; Report of the National Research Council of the National Academies. Explaining Divergent Levels of Longevity in High-Income Countries. Washington, DC: The National Academies Press, 2011.

11. Kones $\mathrm{R}$. Is prevention a fantasy, or the future of medicine? A panoramic view of recent data, status, and direction in cardiovascular prevention. Ther Adv Cardiovasc Dis. 2011;5(1):61-81.

12. Teo KK, Ounpuu S, Hawken S, et al. Tobacco use and risk of myocardial infarction in 52 countries in the INTERHEART study: a case-control study. Lancet. 2006;368(9536):647-658.

13. Kahn R, Robertson RM, Smith R, Eddy D. The impact of prevention on reducing the burden of cardiovascular disease. Circulation. 2008; 118(5):576-585.

14. The Lp-PLA2 Studies Collaboration. Lipoprotein-associated phospholipase A2 and risk of coronary disease, stroke, and mortality: collaborative analysis of 32 studies. Lancet. 2010;375(9725):1536-1544.

15. Kones R. Rosuvastatin, inflammation, C-reactive Protein, JUPITER, and primary prevention of cardiovascular disease - a perspective. Drug Des Devel Ther. 2010;4:383-413.

16. Pearson TA. The prevention of cardiovascular disease: Have we really made progress? Health Aff (Millwood). 2007;26(1):49-60.

17. Hemenway D. Why We Don't Spend Enough on Public Health. New Eng J Med. 2010;362(18):1657-1658.

18. McGlynn EA,Asch SM, Adams J, et al. The quality of health care delivered to adults in the US. $N$ Engl J Med. 2003;348(26):2635-2645.

19. Trust for America's Health. Prevention for a Healthier America: Investments in Disease Prevention Yields Significant Savings, Stronger Communities. Washington, DC: Trust for America's Health; July 2008. Available at: http://healthyamericans.org/reports/prevention08/ Prevention08Exec.pdf. Accessed March 24, 2011.

20. Farley TA, Dalal MA, Mostashari F, Frieden TR. Deaths preventable in the US by improvements in use of clinical preventive services. $\mathrm{Am}$ J Prev Med. 2010;38(6):684-685.

21. National Institute of Health and Clinical Excellence. NICE Public Health Guidance 25: Prevention of Cardiovascular Disease at Population Level. London, UK: National Institute of Health and Clinical Excellence; 2010.

22. Strasser T. Reflections on cardiovascular diseases. Interdiscip Sci Rev. 1978;3:225-230. 
23. Pippin JJ. Primary Prevention Cardiovascular Disease: Better Than Drugs. Arch Intern Med. 2010;170(20):1860-1861.

24. De Lorgeril M, Salen P, Rabeus M. Primary Prevention Cardiovascular Disease: Better Than Drugs - Reply. Arch Intern Med. 2010; 170(20): 1861 .

25. Armstrong ML, Megan MB. Lipid depletion in atheromatous coronary arteries in rhesus monkeys after regression diets. Circ Res. 1972; 30(6):675-680.

26. Ornish D, Scherwitz LW, Billings JH, et al. Intensive lifestyle changes for reversal of coronary heart disease: five-year follow-up of the Lifestyle Heart Trial. JAMA. 1998;280(23):2001-2007.

27. Lloyd-Jones DM, Leip EP, Larson MG, et al. Prediction of lifetime risk for cardiovascular disease by risk factor burden at 50 years of age. Circulation. 2006;113(6):791-798.

28. Kullo IJ, Cooper LT. Early identification of cardiovascular risk using genomics and proteomics. Nat Rev Cardiol. 2010;7(6):309-317.

29. Lavie CJ, Milani RV, Ventura HO. Obesity and cardiovascular disease: risk factor, paradox, and impact of weight loss. $\mathrm{J} \mathrm{Am} \mathrm{Coll} \mathrm{Cardiol}$. 2009;53(21):1925-1932.

30. Ebrahim S, Taylor F, Ward K, Beswick A, Burke M, Davey Smith G. Multiple risk factor interventions for primary prevention of coronary heart disease. Cochrane Database Syst Rev. 2011;(1):CD001561.

31. Graham I, Atar D, Borch-Johnsen K, et al. European guidelines on cardiovascular disease prevention in clinical practice: executive summary: Fourth Joint Task Force of the European Society of Cardiology and Other Societies on Cardiovascular Disease Prevention in Clinical Practice (Constituted by representatives of nine societies and by invited experts). Eur Heart J. 2007;28(19):2375-2414

32. Greenland P, Alpert JS, Beller GA, et al. 2010 ACCF/AHA guideline for assessment of cardiovascular risk in asymptomatic adults: a report of the American College of Cardiology Foundation/American Heart Association Task Force on Practice Guidelines. Developed in collaboration with the American Society of Echocardiography, American Society of Nuclear Cardiology, Society of Atherosclerosis Imaging and Prevention, Society for Cardiovascular Angiography and Interventions, Society of Cardiovascular Computed Tomography, and Society for Cardiovascular Magnetic Resonance. J Am Coll Cardiol. 2010;56(25):2182-2199.

33. Institute of Medicine. Crossing the Quality Chasm: A New Health System for the Twenty-first Century. Washington, DC: National Academy Press; 2001.

34. Brindis R, Spertus JA. President's Page: Employing shared decisionmaking models to improve care and patient value: A cardiovascular Professional initiative. J Am Coll Cardiol. 2010;56(24):2046-2048.

35. White S. Effectiveness of public health and education programs for creating awareness of and managing cardiovascular disease. Patient Intelligence. 2011;3:11-21.

36. Heneghan C. Considerable uncertainty remains in the evidence for primary prevention of cardiovascular disease [editorial]. The Cochrane Library 2011 (19 Jan). Available at: http://www.thecochranelibrary. com/details/editorial/983199/Considerable-uncertainty-remainsin-the-evidence-for-primary-prevention-of-cardi.html. Accessed March 24, 2011.

37. Finucane MM, Stevens GA, Cowan MJ, et al; on behalf of the Global Burden of Metabolic Risk Factors of Chronic Diseases Collaborating Group (Body Mass Index). National, regional, and global trends in body-mass index since 1980: systematic analysis of health examination surveys and epidemiological studies with 960 country-years and 9.1 million participants. Lancet. 2011;377(9765): 557-567.

38. Farzadfar F, Finucane MM, Danaei G, et al; on behalf of the Global Burden of Metabolic Risk Factors of Chronic Diseases Collaborating Group (Cholesterol). National, regional, and global trends in serum total cholesterol since 1980: systematic analysis of health examination surveys and epidemiological studies with 321 country-years and 3.0 million participants. Lancet. 2011;377(9765): $578-586$.
39. Danaei G, Finucane MM, Lin JK, et al; on behalf of the Global Burden of Metabolic Risk Factors of Chronic Diseases Collaborating Group (Blood Pressure). National, regional, and global trends in systolic blood pressure since 1980: systematic analysis of health examination surveys and epidemiological studies with 786 country-years and 5.4 million participants. Lancet. 2011;377(9765):568-577.

40. Anand SS, Yusuf S. Stemming the global tsunami of cardiovascular disease. Lancet. 2011;377(9765):529-532.

41. Capewell S, Hayes DK, Ford ES, et al. Life-years gained among US adults from modern treatments and changes in the prevalence of 6 coronary heart disease risk factors between 1980 and 2000. Am J Epidemiol. 2009;170(2):229-236.

42. Björck L, Rosengren A, Bennett K, Lappas G, Capewell S. Modelling the decreasing coronary heart disease mortality in Sweden between 1986 and 2002. Eur Heart J. 2009;30(9):1046-1056.

43. Young F, Capewell S, Ford ES, Critchley JA. Coronary Mortality Declines in the US Between 1980 and 2000. Quantifying the Contributions from Primary and Secondary Prevention. Amer J Prev Med. 2010;39(3):228-234.

44. USDHHS, National Center for Health Statistics. Second National Health and Nutrition Examination Survey (NHANES II). Hyattsville, MD: CDC;1976-1980.

45. USDHHS, National Center for Health Statistics. Second National Health and Nutrition Examination Survey (Continuous NHANES). Hyattsville, MD: CDC; 1999-2002.

46. Briffa TG, Hobbs MS, Tonkin A, et al. Population trends of recurrent coronary heart disease event rates remain high. Circ Cardiovasc Qual Outcomes. 2011;4(1):107-113.

47. Rose G. The Strategy of Preventive Medicine. Oxford, UK: Oxford University Press; 1992.

48. Rose G. Strategy of prevention: lessons from cardiovascular disease. Br Med J (Clin Res Ed). 1981;282(6279):1847-1851.

49. Rose G. Sick individuals and sick populations. Int J Epidemiol. 1985;14(1):32-38.

50. Rose G. Strategies of prevention: the individual and the population. In: Marmot M, Elliott P, editors. Coronary Heart Disease Epidemiology: From Aetiology to Public Health. Oxford, UK: Oxford University Press; 1991:631-641.

51. Hingorani AD, Psaty BM. Primary Prevention of Cardiovascular Disease. Time to Get More or Less Personal? JAMA. 2009;302(19): 2144-2145.

52. Berger JS, Jordan CO, Lloyd-Jones DM, Blumenthal RS. Screening for cardiovascular risk in asymptomatic patients. $J \mathrm{Am}$ Coll Cardiol. 2010;55(12):1169-1177.

53. Fryar CD, Hirsch R, Eberhardt MS, Yoon SS, Wright JD. Hypertension, high serum total cholesterol, and diabetes: Racial and ethnic prevalence differences in US adults, 1999-2006. NCHS data brief, no 36. Hyattsville, MD: National Center for Health Statistics. April 26, 2010. Available at: http://www.cdc.gov/nchs/default.htm. Accessed March 24, 2011.

54. Roger VL, Go AS, Lloyd-Jones DM, et al; on behalf of the American Heart Association Statistics Committee and Stroke Statistics Committee. Heart Disease and Stroke Statistics-2011 Update: A Report From the American Heart Association. Circulation. 2011;123(4): e18-e209.

55. Paez KA, Zhao L, Hwang W. Rising Out-Of-Pocket Spending For Chronic Conditions: A Ten-Year Trend. Health Aff (Millwood). 2009; 28(1):15-25.

56. Levi J, Vinter S, St. Laurent R, Segal LM. F as in Fat 2010 Finds Continued Rise in Nation's Obesity Rates. Trust for America's Health, and the Robert Wood Johnson Foundation, June 29, 2010. Available at: http:// www.rwjf.org/pr/product.jsp?id=65469. Accessed March 24, 2011.

57. Barnes PM, Heyman KM, Freeman G, Schiller JS. Early release of selected estimates based on data from the 2009 National Health Interview Survey. Hyattsville, MD: National Center for Health Statistics. June 16, 2010. Available at: http://www.cdc.gov/nchs/nhis htm. Accessed March 24, 2011. 
58. National Center for Health Statistics. Health, United States, 2010. CDC. Hyattsville MD 563 p2/16/2011. Available at: http://www.cdc. gov/nchs/data/hus/hus10.pdf. Accessed March 24, 2011.

59. Heart and Stroke Foundation. 2011 Heart and Stroke Foundation Report on Canadians' Health. Denial is putting Canadians at high risk of cutting their lives short. February 1, 2011. Ottawa, ON. Available at: www.heartandstroke.com/atf/cf/\%7B99452d8b-e7f1-4bd6-a57db136ce6c95bf\%7D/lr-FINAL-Reportcard\%20HSF_2011-english.pdf. Accessed March 24, 2011.

60. Huang ES, Basu A, O'grady M, Capretta JC. Projecting the Future Diabetes Population Size and Related Costs For The US. Diab Care. 2009;32(12):2225-2229.

61. Boyle JP, Thompson TJ, Gregg EW, Barker LE, Williamson DF. Projection of the year 2050 burden of diabetes in the US adult population: dynamic modeling of incidence, mortality, and prediabetes prevalence. Population Health Metrics. 2010;8:29.

62. Yancy CW. Is Ideal Cardiovascular Health Attainable? Circulation. 2011;123(8):835-837.

63. Kones R. Low-fat versus low-carbohydrate diets, weight loss, vascular health, and prevention of coronary artery disease: the evidence, the reality, the challenge, and the hope. Nutr Clin Pract. 2010;25(5): 528-541.

64. Heber D. An integrated view of obesity. Am J Clin Nutr. 2010;91(1): 280S-283S.

65. Bray GA. Let's treat obesity seriously. Amer Fam Physician. 2010; 81(12):1449-1455.

66. Number of Americans with Diabetes Rises to Nearly 26 Million. More than a third of adults estimated to have prediabetes. CDC Press Release, January 13, 2011. Available at: http://www.cdc.gov/ media/releases/2011/p0126_diabetes.html. Accessed March 24, 2011.

67. Danaei G, Friedman AB, Oza S, Murray C JL, Ezzati M. Diabetes prevalence and diagnosis in US states: analysis of health surveys. Popul Health Metr. 2009;7:16.

68. Hammond RA, Levine R. The economic impact of obesity in the United States. Diabetes Metab Syndr Obes. 2010;3:285-295.

69. Vickers MH, SLoboda DM. Prenatal nutritional influences on obesity risk in offspring. Nutrition and Dietary Supplements. 2010;2:137-149.

70. Ford ES, Li C, Zhao G, Tsai J. Trends in obesity and abdominal obesity among adults in the United States from 1999-2008. Int J Obes (Lond). 2011;35:736-743.

71. Bambs C, Kip KE, Dinga A, Mulukutla SR, Aiyer AN, Reis SE. Low Prevalence of "Ideal Cardiovascular Health" in a Community-Based Population: The Heart Strategies Concentrating on Risk Evaluation (Heart SCORE) Study. Circulation. 2011;123(8):850-857.

72. CDC National Center for Health Statistics. CDC mortality data, 2008: CDC latest release mortality data, 2005. Available at: www.cdc.gov/ nchs/fastats/deaths.htm.

73. Sacker A, Head J, Bartley M. Impact of coronary heart disease on health functioning in an aging population: are there differences according to socioeconomic position? Psychosom Med. 2008;70(2):133-140.

74. Allender S, Scarborough P, Peto V, et al. European cardiovascular disease statistics 2008. European Heart Network, 2008. Available at: www.heartstats.org/uploads/documents\%5Cproof30 NOV2007.pdf.

75. WHO Statistical Information System. Mortality data for ICD 10 codings. Available at: www.who.int/whosis/database/mort/download/ $\mathrm{ftp} /$ morticd10.zip.

76. Ford ES, Capewell S. Coronary heart disease mortality among young adults in the US from 1980 to 2002: concealed leveling or mortality rates. J Am Coll Cardiol. 2007;50(22):2128-2132.

77. Ayer J, Steinbeck K. Placing the cardiovascular risk of childhood obesity in perspective. Int J Obes. 2010;34(1):4-5.

78. Berenson G. Cardiovascular health promotion for children: A model for a Parish (County)-wide program (implementation and preliminary results. Prev Cardiol. 2010;13(1):23-28.
79. Prevalence of abnormal lipid levels among youths - United States, 1999-2006. Morbidity and Mortality Weekly Report (MMWR). 2010;59(02):29-33. January 22, 2010. Available at: http://www.cdc. gov/mmwr/preview/mmwrhtml/mm5902a1.htm\#. Accessed March 24, 2011.

80. Berenson GS, Srinivasan SR. Cardiovascular Risk in Young Persons: Secondary or Primordial Prevention? Ann Int Med. 2010;153(3): 202-203.

81. Pletcher MJ, Bibbins-Domingo K, Liu K, et al. Nonoptimal lipids commonly present in young adults and coronary calcium later in life: The CARDIA (Coronary Artery Risk Development in Young Adults) Study. Ann Intern Med. 2010;153(3):137-146.

82. Falk E, Naghavi M, Shah PK. Legislating screening for atherosclerosis. JAMA. 2008;299(18):2147-2148.

83. Gillespie C, Kuklina EV, Briss PA, Blair NA, Hong Y. Vital signs: prevalence, treatment, and control of hypertension - United States, 1999-2002 and 2005-2008. MMWR. 2011;60(04);103-108. Available at: http://www.cdc.gov/mmwr/preview/mmwrhtml/mm60e0201a1.htm Accessed March 24, 2011.

84. Kuklina E, Shaw KM, Y Hong Y. Vital signs: prevalence, treatment, and control of high levels of low-density lipoprotein cholesterol - United States, 1999-2002 and 2005-2008. MMWR. 2011;60(04);109-114. Available at: http://www.cdc.gov/mmwr/preview/mmwrhtml/ mm60e0201a2.htm. Accessed March 24, 2011.

85. Katch $\mathrm{H}, \mathrm{Mead} \mathrm{H}$. The role of self-efficacy in cardiovascular disease self-management: a review of effective programs. Patient Intelligence. 2010;2:33-44.

86. Bosworth HB. Challenges and strategies to improve patient health literacy and competencies. Patient Intelligence. 2010;2:19-25.

87. McNeal TM, Colbert CY, Cable C, et al. Patients' attention to and understanding of adverse drug reaction warnings. Patient Intelligence. 2010;2:59-68.

88. Laufs U, Rettig-Ewen V, Böhm M. Strategies to improve drug adherence. Eur Heart J. 2011;32(3):264-268.

89. Fischer MA, Stedman MR, Lii J, et al. Primary Medication NonAdherence: Analysis of 195,930 Electronic Prescriptions. J Gen Int Med. 2010;25(4):284-290.

90. Lardizabal JA, Deedwania PC. Benefits of statin therapy and compliance in high risk cardiovascular patients. Vasc Health Risk Manag. 2010;6:843-853.

91. Ho PM, Magid DJ, Shetterly SM, et al. Medication nonadherence isassociated with a broad range of adverse outcomes in patients with coronary artery disease. Am Heart J. 2008;155(4): 772-729.

92. Donnelly LA, Doney AS, Morris AD, Palmer CN, Connan PT. Long-term adherence to statin treatment in diabetes. Diabet Med. 2008; 25(7):850-855.

93. Kotseva K, Wood D, De Backer G, et al. EUROASPIRE Study Group. Cardiovascular prevention guidelines in daily practice: a comparison of EUROASPIRE I, II, and III surveys in eight European countries. Lancet. 2009;373(9667):929-940.

94. Popkin BM. Recent dynamics suggest selected countries catching up to US obesity. Am J Clin Nutr. 2010;91(1):284S-288S.

95. Shaw JE, Sicree RA, Zimmet PZ. Global estimates of the prevalence of diabetes for 2010 and 2030. Diabetes Res Clin Pract. 2010;87(1): 4-14.

96. Zhang P, Zhang X, Brown J, et al. Global healthcare expenditure on diabetes for 2010 and 2030. Diabetes Res Clin Pract. 2010;87(3): 293-301.

97. Simmons RK, Unwin N, Griffin SJ. International Diabetes Federation: An update of the evidence concerning the prevention of type 2 diabetes. Diabetes Res Clin Pract. 2010;87(2):143-149.

98. Joshi R, Jan S, Wu Y, MacMahon S. Global inequalities in access to cardiovascular health care: our greatest challenge. J Am Coll Cardiol. 2008;52(23):1817-1825. 
99. Yusuf S, Hawken S, Ounpuu S, et al. Effect of potentially modifiable risk factors associated with myocardial infarction in 52 countries (the INTERHEART study): case-control study. Lancet. 2004;364(9438): 937-952.

100. Reddy KS, Prabhakaran D, Jeemon P, et al. Educational status and cardiovascular risk profile in Indians. Proc Natl Acad Sci U S A. 2007;104:16263-16268.

101. Franco M, Cooper RS, Bilal U, Fuster V. Challenges and opportunities for cardiovascular disease prevention. Amer J Med. 2011;124(2):95-102.

102. Kavey E-RW, Daniels SR, Lauer RM, Atkins DL, Hayman LL, Taubert K. American Heart Association guidelines for primary prevention of atherosclerotic cardiovascular disease beginning in childhood. Circulation. 2003;107(11):1562-1566.

103. McMahan CA, Gidding SS, Viikari JSA, et al. Association of Pathobiologic determinants of atherosclerosis in youth risk score and 15 -year change in risk score with carotid artery intima-media thickness in young adults (from the Cardiovascular Risk in Young Finns Study). Am J Cardiol. 2007;100(7):1124-1129.

104. Shah AS, Dolan LM, Kimball TR, et al. Influence of duration of diabetes, glycemic control, and traditional cardiovascular risk factors on early atherosclerotic vascular changes in adolescents and young adults with type 2 diabetes mellitus. J Clin Endocrinol Metab. 2009;94(10):3740-3745.

105. Newman WP, Wattigney W, Berenson GS. Autopsy studies in United States children and adolescents: relationship of risk factors to atherosclerotic lesions. Ann NY Acad Sci. 1991;623:16-25.

106. Strong JP, Malcom GT, McMahan CA, et al. Prevalence and extent of atherosclerosis in adolescents and young adults: implications for prevention from the Pathobiological Determinants of Atherosclerosis in Youth Study. JAMA. 1999;281(8):727-735.

107. Corvalán C, Uauy R, Kain J, Martorell R. Obesity indicators and cardiometabolic status in 4-y-old children. Am J Clin Nutr. 2010; 91(1):166-174

108. Mauras N, DelGiorno C, Kollman C, et al. Obesity without established comorbidities of the metabolic syndrome is associated with a proinflammatory and prothrombotic state, even before the onset of puberty in children. J Clin Endocrinol Metab. 2010,95(3): 1060-1068.

109. Milei J, Ottaviani G, Lavezzi AM, Grana DR, Stella I, Matturi L. Perinatal and infant early atherosclerotic coronary lesions. Can J Cardiol. 2008;24(2):137-141.

110. Tuzcu EM, Kapadia SR, Tutar E, et al. High prevalence of coronary atherosclerosis in asymptomatic teenagers and young adults: evidence from intravascular ultrasound. Circulation. 2001;103(22): 2705-2710.

111. Enos WF, Holmes RH, Beyer J. Coronary disease among United States soldiers killed in action in Korea: preliminary report. JAMA. 1953; 552(12):1090-1093.

112. McNamara JJ, Molot MA, Stremple JF, Cutting RT. Coronary artery disease in combat casualties in Vietnam. JAMA. 1971;216(7) 1185-1887.

113. Newman WP, Freedman DS, Voors AW, et al. Relation of serum lipoprotein levels and systolic blood pressure to early atherosclerosis: the Bogalusa Heart Study. N Engl J Med. 1986;314(3):138-144.

114. Berenson GS, Srinivasan SR, Bao W, Newman WP 3rd, Tracy RE, Wattigney WA. Association between multiple cardiovascular risk factors and atherosclerosis in children and young adults: the Bogalusa Heart Study. N Engl J Med. 1998;338(23):1650-1656.

115. Nguyen QMN, Srinivasan SR, Xu J-H, Chen W, Kieltyka L, Berenson GS. Utility of childhood glucose homeostasis variables in predicting adult diabetes and related cardiometabolic risk factors. Diabetes Care. 2010;33(3):670-675.

116. Berenson GS, Wattigney WA, Tracy RE, et al. Atherosclerosis of the aorta and coronary arteries and cardiovascular risk factors in persons aged 6 to 30 years and studied at necropsy (The Bogalusa Heart Study). Am J Cardiol. 1992;70(9):851-858.
117. Berenson GS. Cardiovascular risk begins in childhood. A time for action. Am J Prev Med. 2009;37(1 Suppl):S1-S2.

118. Twisk JW, Kemper HC, Mellenbergh GJ. Mathematical and analytical aspects of tracking. Epidemiol Rev. 1994;16(2):165-182.

119. García RG, Pérez M, Maas R, Schwedhelm E, Boger RH, Lopez-Jaramillo P. Plasma concentrations of asymmetric dimethylarginine (ADMA) in metabolic syndrome. Int J Cardiol. 2007;122(2): $176-178$.

120. Gillum RF. Association of serum C-reactive protein and body fat distribution and overweight in Mexican American children. $J$ Natl Med Assoc. 2003;95(7):545-552.

121. López-Jaramillo P, Herrera E, García RG, Camacho PA, Castillo VR. Relationship of body mass index, C-reactive protein and blood pressure in a Hispanic pediatric population. Am J Hypertens. 2008; 21(5):527-532.

122. Magnussen CG, Thomson R, Cleland VJ, Ukoumunne OC, Dwyer T, Venn A. Factors affecting the stability of blood lipid and lipoprotein levels from youth to adulthood: evidence from the Childhood Determinants of Adult Health Study. Arch Pediatr Adolesc Med. 2011;165(1) 68-76.

123. PDAY Research Group. Relationship of atherosclerosis in young men to serum lipoprotein cholesterol concentrations and smoking: a preliminary report from the Pathological Determinants of Atherosclerosis in Youth (PDAY) Research Group. JAMA. 1990;264(23):3018-3024.

124. Wissler RW, Strong JP; the PDAY Research Group: Risk factors and progression of atherosclerosis in youth. Am J Pathol. 1998;153(4): 1023-1033.

125. McGill HC Jr, McMahan CA, Gidding SS. Preventing heart disease in the 21st century: implications of the Pathobiological Determinants of Atherosclerosis in Youth (PDAY) Study. Circulation. 2008;117(9): 1216-1227.

126. McGill HC, McMahan CA. Starting early to control all risk factors in order to prevent coronary heart disease. Clinical Lipidol. 2010; 5(1):87-93.

127. Juonala M, Viikari JSA, Kähönen M, et al. Life-time risk factors and progression of carotid atherosclerosis in young adults: the Cardiovascular Risk in Young Finns study. Eur Heart J. 2010;31(14): 1745-1751.

128. Juonala M, Magnussen CG, Venn A, et al. Influence of age on associations between childhood risk factors and carotid intimamedia thickness in adulthood: The Cardiovascular Risk in Young Finns Study, the Childhood Determinants of Adult Health Study, the Bogalusa Heart Study, and the Muscatine Study for the International Childhood Cardiovascular Cohort (i3C) Consortium. Circulation. 2010;122(24):2514-2520.

129. Gidding SS. Assembling evidence to justify prevention of atherosclerosis beginning in youth. Circulation. 2010;122(24):2493-2494.

130. Daniels SR, Greer FR, Committee on Nutrition. Lipid screening and cardiovascular health in childhood. Pediatrics. 2008;122(1): 198-208.

131. O’Gorman CSM, O’Neill MB, Conwell LS. Considering statins for cholesterol-reduction in children if lifestyle and diet changes do not improve their health: a review of the risks and benefits. Vasc Health Risk Manag. 2011;7:1-14.

132. Cohen H, Stein-Zamir C, Hamiel O, et el. Israeli guidelines for the management of hypercholesterolemia in children and adolescents Report of the pediatric association expert group. e-SPEN. Eur e-Journal Clin Nutr Metabol. 2010;5(3):e132-e143.

133. Iughetti L, Bruzzi P, Predieri B. Evaluation and management of hyperlipidemia in children and adolescents. Curr Opin Ped. 2010;22:(4):485-493.

134. Gooding HC, D de Ferranti S. Cardiovascular risk assessment and cholesterol management in adolescents: getting to the heart of the matter. Curr Opin Ped. 2010;22(4):398-404.

135. Wierzbicki AS, Viljoen A. Hyperlipidaemia in paediatric patients: the role of lipid-lowering therapy in clinical practice. Drug Safety. 2010;33(2):115-125. 
136. Lebenthal Y, Horvath A, Dziechciarz P, Szajewska H, Shamir R. Are treatment targets for hypercholesterolemia evidence based? Systematic review and meta-analysis of randomised controlled trials. Arch Dis Child. 2010;95(9):673-680.

137. De Ferranti S, Ludwig DS. Storm over Statins - The Controversy Surrounding Pharmacologic Treatment of Children. N Engl J Med. 2008;359(13):1309-1312.

138. Steinberg D, Glass CK, Witztum JL. Evidence mandating earlier and more aggressive treatment of hypercholesterolemia. Circulation. 2008;118(6):672-677.

139. Simell O, Niinikoski H, Ronnemaa T, et al. Cohort Profile: The STRIP Study (Special Turku Coronary Risk Factor Intervention Project), an infancy-onset dietary and life-style intervention trial. Int J Epidemiol. 2009;38(3):650-655.

140. Kuklina EV, Yoon PW, Keenan NL. Prevalence of coronary heart disease risk factors and screening for high cholesterol levels among young adults, United States, 1999-2006. Ann. Fam Med. 2010;8(4):327-333.

141. Gidding SS, Lichtenstein AH, Faith MS, et al. Implementing American Heart Association Pediatric and Adult Nutrition Guidelines: A Scientific Statement From the American Heart Association Nutrition Committee of the Council on Nutrition, Physical Activity and Metabolism, Council on Cardiovascular Disease in the Young, Council on Arteriosclerosis, Thrombosis and Vascular Biology, Council on Cardiovascular Nursing, Council on Epidemiology and Prevention, and Council for High Blood Pressure Research. Circulation. 2009;119(8):1161-1175.

142. Broyles S, Katzmarzyk PT, Srinivasan SR, et al. The pediatric obesity epidemic continues unabated in Bogalusa, Louisiana. Pediatrics. 2010;125(5):900-905.

143. US Preventive Services Task Force. Screening for obesity in children and adolescents: US Preventive Services Task Force recommendation statement. Pediatrics. 2010;125(2):361-367.

144. National Center for Chronic Disease Prevention and Health Promotion: Healthy Youth! Available at: http://www.cdc.gov/HealthyYouth. Accessed May 16, 2011.

145. August G. Prevention and treatment of pediatric obesity: an Endocrine Society clinical practice guidelines based on expert opinion. J Clin Endocrinol Metab. 2008;93(12):4576-4599.

146. Kannel WB, McGee D, Gordon T. A general cardiovascular risk profile: the Framingham Study. Am J Cardiol. 1976;38(1): $46-51$.

147. Gordon T, Kannel WB. Multiple risk functions for predicting coronary heart disease: the concept, accuracy, and application. Am Heart J. 1982;103(6):1031-1039.

148. Anderson M, Wilson PW, Odell PM, Kannel WB. An updated coronary risk profile: a statement for health professionals. Circulation. 1991; 83(1):356-362.

149. Wilson PWF, D'Agostino RB, Levy D, Belanger AM, Silbershatz H, Kannel WB. Prediction of coronary heart disease using risk factor categories. Circulation. 1998;97(18):1837-1847.

150. Conroy RM, Pyorala K, Fitzgerald AP, et al. Estimation of ten-year risk of fatal cardiovascular disease in Europe: the SCORE project. Eur Heart J. 2003;24(11):987-1003.

151. Hippisley-Cox J, Coupland C, Vinogradova Y, Robson J, May M, Brindle P. Derivation and validation of QRISK, a new cardiovascular disease risk score for the United Kingdom: prospective open cohort study. BMJ. 2007;335(7611):136-147.

152. Woodward M, Brindle P, Tunstall-Pedoe H. Adding social deprivation and family history to cardiovascular risk assessment: the ASSIGN score from the Scottish Heart Health Extended Cohort (SHHEC). Heart. 2007;93(2):172-176.

153. Third Report of the National Cholesterol Education Program (NCEP) Expert Panel on Detection, Evaluation, and Treatment of High Blood Cholesterol in Adults (Adult Treatment Panel III) final report. Circulation. 2002;106(25):3143-3421.
154. D'Agostino Sr RB, Grundy S, Sullivan LM, Wilson P, for the CHD Risk Prediction Group. Validation of the Framingham coronary disease prediction scores. results of a multiple ethnic groups investigation. JAMA. 2001;286(2):180-187.

155. Assmann G, Cullen P, Schulte H. Simple scoring scheme for calculating the risk of acute coronary events based on the 10-year follow-up of the Prospective Cardiovascular Münster (PROCAM) study. Circulation. 2002;105(3):310-315.

156. Ridker PM, Buring JE, Rifai N, Cook NR. Development and validation of improved algorithms for the assessment of global cardiovascular risk in women: the Reynolds Risk Score. JAMA. 2007;297(6): 611-619.

157. Ridker PM, Paynter NP, Rifai N, Gaziano JM, Cook NR. C-reactive protein and parental history improve global cardiovascular risk prediction: the Reynolds risk score for men. Circulation. 2008; 118(22):2243-2251.

158. Pearson TA, Blair SN, Daniels SR, et al. AHA guidelines for primary prevention of cardiovascular disease and stroke: 2002 update: consensus panel guide to comprehensive risk reduction for adult patients without coronary or other atherosclerotic vascular diseases. Circulation. 2002;106(3):388-391.

159. Guide to Clinical Preventive Services 2007: Recommendations of the US Preventive Services Task Force. Agency for Healthcare Research and Quality Pub. No. 07-05100. September 2007. Available at: https://www.oxhp.com/secure/materials/member/adult_preventive. pdf. Accessed March 24, 2011.

160. De Backer G, Ambrosioni E, Borch-Johnsen K, et al. European guidelines on cardiovascular disease and prevention in clinical practice. Atherosclerosis. 2003;171(1):145-155.

161. De Backer GG. Risk factors and prevention of cardiovascular disease: a review. Dialogues Cardiovasc Med. 2008;13(2):83-99.

162. Dent THS. Predicting the risk of coronary heart disease: I. The use of conventional risk markers. Atherosclerosis. 2010;213(2)345-351.

163. Nasir K, Budoff MJ, Wong ND, et al. Family history of premature coronary heart disease and coronary artery calcification: Multi-Ethnic Study of Atherosclerosis (MESA). Circulation. 2007;116(6): 619-626.

164. Parikh NI, Hwang S-J, Larson MG, et al. Parental occurrence of premature cardiovascular disease predicts increased coronary artery and abdominal aortic calcification in the Framingham Offspring and Third Generation Cohorts. Circulation. 2007;116(13):1473-1481.

165. Lloyd-Jones DM, Nam B-H, D'Agostino RB Sr, et al. Parental cardiovascular disease as a risk factor for cardiovascular disease in middle-aged adults: a prospective study of parents and offspring. JAMA. 2004;291(18):2204-2211.

166. Pohjola-Sintonen S, Rissanen A, Liskola P, and Luomanmäki K. Family history as a risk factor of coronary heart disease in patients under 60 years of age. Eur Heart J. 1998;19(2):235-239.

167. Younger JF. Family history is important in estimating coronary risk. BMJ. 2000;321(7258):448.

168. Lloyd-Jones DM, Dyer AR, Wang R, Daviglus ML, Greenland P. Risk factor burden in middle age and lifetime risks for cardiovascular and non-cardiovascular death (Chicago Heart Association Detection Project in Industry). Am J Cardiol. 2007;99(4):535-540.

169. D’Agostino RB Sr, Vasan RS, Pencina MJ, et al. General cardiovascular risk profile for use in primary care: the Framingham Heart Study. Circulation. 2008;117(6):743-753.

170. De Koning JS, Klazinga NS, Koudstaal PJ, et al. Quality of care in stroke prevention: results of an audit study among general practitioners. Prev Med. 2004;38(2):129-136.

171. De Muylder R, Lorant V, Paulus D, et al. Obstacles to cardiovascular prevention in general practice. Acta Cardiol. 2004;59(2):119-125.

172. Eaton CB, Galliher JM, McBride PE, et al. Family physician's knowledge, beliefs, and self-reported practice patterns regarding hyperlipidemia: a National Research Network (NRN) survey. J Am Board Fam Med. 2006;19(1):46-53. 
173. Graham IM, Stewart M, Hertog MG. Factors impeding the implementation of cardiovascular prevention guidelines: findings from a survey conducted by the European Society of Cardiology. Eur J Cardiovasc Prev Rehabil. 2006;13(5):839-845.

174. Fuster V. Are predictive risk scores useful? Nat Rev Cardiol. 2009; 6(8):493.

175. Gupta M, Kajil M, Hirjikaka S, et al. Accuracy of Cardiovascular (CV) Risk Stratification by Canadian Primary Care Physicians: Preliminary Results From the Primary Care Audit of Global Risk Management (PARADIGM) Study, Abstract 16946. Circulation. 2010;122(21 Suppl):A16946.

176. Sheridan SL, Crespo E. Does the routine use of global coronary heart disease risk scores translate into clinical benefits or harms? A systematic review of the literature. BMC Health Serv Res. 2008; 8:60.

177. Kones R. Recent advances in the management of chronic stable angina I: Approach to the patient, diagnosis, pathophysiology, risk stratification, and gender disparities. Vasc Health Risk Manag. 2010;6:635-656.

178. Madhok V, Fahey T. Cardiovascular risk estimation: important but may be inaccurate. BMJ. 2006;332(7555):1422.

179. Akosah KO, Schaper A, Cogbill C, Schoenfeld P. Preventing myocardial infarction in the young adult in the first place: how do the National Cholesterol Education Panel III guidelines perform? J Am Coll Cardiol. 2003;41(9):1475-1479.

180. National Center for Health Statistics. Third National Health and Nutrition Examination Survey III. Public-Use Data files. Available at: http:// www.cdc.gov/nchs/nhanes/nh3data.htm. Accessed May 16, 2011.

181. Cavanaugh-Hussey MW, Berry JD, Lloyd-Jones DM. Who exceeds ATP-III risk thresholds? Systematic examination of the effect of varying age and risk factor levels in the ATP-III risk assessment pool. Prev Med. 2008;47(6):619-623.

182. Feeman W Jr. BGS graph predicts better than the Framingham Risk Score. J Clin Lipidol. 2010;4(3):203.

183. Cooper JA, Miller GJ, Humphries SE. A comparison of the PROCAM and Framingham point-scoring systems for estimation of individual risk of coronary heart disease in the Second Northwick Park Heart Study. Atherosclerosis. 2005;181(1):93-100.

184. Orford JL, Sesso HD, Stedman M, et al. A comparison of the Framingham and European Society of Cardiiology coronary heart disease risk prediction models in the same normative aging study. Am Heart J. 2002;144(1):95-100.

185. Lloyd-Jones DM. Cardiovascular risk prediction. Basic concepts, current status, and future directions. Circulation. 2010;121(15):1768-1777.

186. Vasan RS, Sullivan LM, Wilson PW, et al. Relative importance of borderline and elevated levels of coronary heart disease risk factors. Ann Intern Med. 2005;142(6):393-402.

187. Berry JD, Liu K, Folsom AR, et al. Prevalence and progression of subclinical atherosclerosis in younger adults with low short-term but high lifetime estimated risk for cardiovascular disease: the CARDIA and MESA studies. Circulation. 2009;119(3):382-389.

188. Mosca L, Banka CL, Benjamin EJ, et al. Evidence-based guidelines for cardiovascular disease prevention in women: 2007 update. Circulation. 2007;115(11):1481-1501.

189. Rohatgi AK, Khera A. The Upcoming ATP IV Guidelines. Lipid Spin. 2010;8(4):6-9.

190. Genest J, McPherson R, Frohlich J, et al. 2009 Canadian Cardiovascular Society/Canadian guidelines for the diagnosis and treatment of dyslipidemia and prevention of cardiovascular disease in the adult - 2009 recommendations. Can J Cardiol. 2009;25(10):567-579.

191. Ferrario CM, Moore MA, Bestermann W Jr, et al. COSEHC global vascular risk management quality improvement program: rationale and design. Vasc Health Risk Manag. 2010,6:1135-1145.

192. Min JK, Shaw LJ, Devereaux RB, et al. Progostic value of multidetector coronary computed tomographic angiography for prediction of all-cause mortality. J Am Coll Cardiol. 2007;50(12):1161-1170.
193. Johnson KM, Dowe DA, Brink JA. Traditional clinical risk assessment tools do not accurately predict coronary atherosclerotic plaque burden: a CT angiography study. Am J Roentgenol. 2009;192(1): 235-243.

194. Johnson KM, Dowe DA. The detection of any coronary calcium outperforms Framingham Risk Score as a first step in screening for coronary atherosclerosis. Am J Roentgenol. 2010;194(5): 1235-1243.

195. Hecht HS. The deadly double standard. The saga of screening for subclinical atherosclerosis. Am J Cardiol. 2008;101(12): 1805-1807.

196. Cooney MT, Dudina AL, Graham IM. Value and limitations of existing scores for the assessment of cardiovascular risk. J Am Coll Cardiol. 2009;54(14):1209-1227.

197. Cooney MT, Dudina A, D’Agostino R, Graham IM. Cardiovascular risk-estimation systems in primary prevention. Do they differ? Do they make a difference? Can we see into the future? Circulation. 2010;122(3):300-310.

198. Shah PK. Screening asymptomatic subjects for subclinical atherosclerosis. Can we, does it matter, and should we? J Am Coll Cardiol. 2010;56(2):98-105.

199. Lauer MS. Screening asymptomatic subjects for subclinical atherosclerosis. Not so obvious. J Am Coll Cardiol. 2010;56(2): 106-108.

200. Lord SJ, Irwig L, Simes RJ. When is measuring sensitivity and specificity sufficient to evaluate a diagnostic test, and when do we need randomized trials? Ann Intern Med. 2006;144(11):850-855.

201. Lloyd-Jones DM, Liu K, Tian L, Greenland P. Narrative review: assessment of C-reactive protein in risk prediction for cardiovascular disease. Ann Intern Med. 2006;145(1):35-42.

202. Lloyd-Jones DM, Tian L. Predicting cardiovascular risk. So what do we do now? Arch Intern Med. 2006;166(13):1342-1344.

203. Dent THS. Predicting the risk of coronary heart disease. II: The role of novel molecular biomarkers and genetics in estimating risk, and the future of risk prediction. Atherosclerosis. 2010;213(2):352-362.

204. Wang TJ, Gona P, Larson MG, et al. Multiple biomarkers for the prediction of first major cardiovascular events and death. $N$ Eng $J$ Med. 2006;355(25):2631-2639.

205. Zethelius B, Berglund L, Sundstrom J, et al. Use of multiple biomarkers to improve the prediction of death from cardiovascular causes. N Engl J Med. 2008;358(20):2107-2116.

206. De Lemos JA, Rohatgi A. Separating the contenders from the pretenders: competitive high-throughput biomarker screening in large population-based studies. Circulation. 2010;121(22):2381-2383.

207. Melander O, Newton-Cheh C, Almgren P, et al. Novel and conventional biomarkers for prediction of incident cardiovascular events in the community. JAMA. 2009;302(1):49-57.

208. Blankenberg S, Zeller T, Saarela O, et al. for the MORGAM Project. Contribution of 30 biomarkers to 10 -year cardiovascular risk estimation in 2 population cohorts: The MONICA, risk, genetics, archiving, and monograph (morgam) biomarker project. Circulation. 2010;121(22):2388-2397.

209. Arsenault BJ, Barter P, DeMicco DA, et al for the TNT Study Investigators. Prediction of cardiovascular events in statin-treated stable coronary patients by lipid and nonlipid biomarkers. J Am Coll Cardiol. 2011;57(1):63-69.

210. Berry JD, Dyer A, Carnethon M, Trian L, Greenland P, Lloyd-Jones DM. Association of traditional risk factors with cardiovascular death across 0 to 10,10 to 20 , and $>20$ years follow-up in men and women. Am J Cardiol. 2008;101(1):89-94

211. Persell SD, Zei C, Cameron KA, Zielinski M, Lloyd-Jones DM. Potential use of 10-year and lifetime coronary risk information for preventive cardiology prescribing decisions: a primary care physician survey. Arch Int Med. 2010;170(5):470-477.

212. Lloyd-Jones DM, Wilson PWF, Larson MG, et al. Framingham risk score and prediction of lifetime risk for coronary heart disease. Am J Cardiol. 2004;94(1):20-24. 
213. Marma AK, Berry JD, Ning H, Persell SD, Lloyd-Jones DM. Distribution of 10-Year and Lifetime Predicted Risks for Cardiovascular Disease in US Adults: Findings From the National Health and Nutrition Examination Survey 2003 to 2006. Circ Cardiovasc Qual Outcomes. 2010;3(1):8-14.

214. Crouse JR III, Bots ML, Evans GW, et al. Does baseline carotid intimamedia thickness modify the effect of rosuvastatin when compared with placebo on carotid intima-media thickness progression? The METEOR study. Eur J Cardiovasc Prev Rehabil. 2010;17(2):223-229.

215. Reiner AP, Carlson CS, Thyagarajan B, et al. Soluble P-selectin, SELP polymorphisms, and atherosclerotic risk in European-American and African-African young adults: the Coronary Artery Risk Development in Young Adults (CARDIA) Study. Arterioscler Thromb Vasc Biol. 2008;28(8):1549-1555.

216. Loria CM, Liu K, Lewis CE, et al. Early adult risk factor levels and subsequent coronary artery calcification: the CARDIA study. $J \mathrm{Am}$ Coll Cardiol. 2007;49(20):2013-2020.

217. Carr JJ, Nelson JC, Wong ND, et al. Calcified coronary artery plaque measurement with cardiac ct in population-based studies: standardized protocol of Multi-Ethnic Study of Atherosclerosis (MESA) and Coronary Artery Risk Development in Young Adults (CARDIA) Study. Radiology. 2005;234(1):35-43.

218. Bild DE, Bluemke DA, Burke GL, et al. Multi-ethnic study of atherosclerosis: objectives and design. Am J Epidemiol. 2002;156(9): 871-881.

219. Folsom AR, Kronmal RA, Detrano RC, et al. Coronary artery calcification compared with carotid intima-media thickness in the prediction of cardiovascular disease incidence: the Multi-Ethnic Study of Atherosclerosis (MESA). Arch Intern Med. 2008;168(12): 1333-1339.

220. Pankaj GP, Raju P, Sondej E, Rodrigues E, Davis G. Use of the Joint British Society cardiovascular risk calculator before initiating statins for primary prevention in hospital medicine: experience from a large university teaching hospital. Int J Gen Med. 2010;3:379-382.

221. Blaha M, Nasir K, DeFilippis A, et al. Association between hsCRP $\geq 2$, Coronary Calcium, and Adverse Events - Implications for the JUPITER Population in the Multi-Ethnic Study of Atherosclerosis (MESA). American Heart Association Scientific Sessions. Nov 17, 2010. Abstract 12825. Summary available at: http://www.theheart.org/ article/1154871.do.

222. Roth GA, Finh SD, Mokdad AH, Aekplakorn W, Hasegawae T, Lim SS. High total serum cholesterol, medication coverage and therapeutic control: an analysis of national health examination survey data from eight countries. Bull World Health Organ. 2011;89(2):92-101.

223. Min JK, Lin FY, Gidseg DS, et al. Determinants of coronary calcium conversion among patients with a normal coronary calcium scan: what is the "warranty period" for remaining normal? $\mathrm{J} \mathrm{Am} \mathrm{Coll} \mathrm{Cardiol.}$ 2010;55(11):1110-1117.

224. Kim KP, Einstein AJ, de González AB. Coronary artery calcification screening: estimated radiation dose and cancer risk. Arch Intern Med. 2009;169(13):1188-1194.

225. Smith-Bindman R, Lipson J, Marcus R, et al. Dose associated with common computed tomography examinations and the associated lifetime attributable risk of cancer. Arch Intern Med. 2009;169(22): 2078-2086.

226. Redberg RF. Cancer risks and radiation exposure from computed tomographic scans: how can we be sure that the benefits outweigh the risks? Arch Intern Med. 2009;169(22):2049-2050.

227. Nambi V, Ballantyne CM: "Risky business": ten years is not a lifetime. Circulation. 2009;119(3):362-364.

228. Nambi V, Chambless L, Folsom AR, et al. Are all patients considered "low risk" for coronary heart disease really low risk? An analysis from the Atherosclerosis Risk in Communities (ARIC) Study. Circulation. 2008;118(Suppl 2):S1125.

229. Nissen SE, Tuzcu EM, Schoenhagen P, et al. Effect of intensive compared with moderate lipid-lowering therapy on progression of coronary atherosclerosis: a randomized controlled trial. JAMA. 2004; 291(9):1071-1080.
230. Nissen SE, Nicholls SJ, Sipahi I, et al. Effect of very high-intensity statin therapy on regression of coronary atherosclerosis: the ASTEROID trial. JAMA. 2006;295(13):1556-1565.

231. Artinian NT, Fletcher GJ, Mozaffarian D, et al. on behalf of the American Heart Association Prevention Committee of the Council on Cardiovascular Nursing. Interventions to promote physical activity and dietary lifestyle changes for cardiovascular risk factor reduction in adults: a scientific statement from the American Heart Association. Circulation. 2010;122(4):406-441.

232. Yancy CW. Transformation of cardiovascular health: presidential address at the American Heart Association 2009 scientific sessions. Circulation. 2010;121(25):2773-2776.

233. Baigent C, Keech A, Kearney PM, et al; Cholesterol Treatment Trialists' (CTT) Collaborators. Efficacy and safety of cholesterollowering treatment. Lancet. 2005;366(9493):1267-1278.

234. Saely CH, Drexel H, Huber K. High-dose statin therapy for high-risk patients. Herz (Hochdosierte Statintherapie für kardiovaskuläre Risikopatienten). 2010;35(7):497-502.

235. Cheung BM, Lam KS. Is intensive LDL-cholesterol lowering beneficial and safe? Lancet. 2010;376(9753):1622-1624.

236. Nissen S. The "fiction" of primary prevention: role of IVUS in identifying early atherosclerosis. April 23, 2004. Available at: http://www. theheart.org/article/216595.do. Accessed March 24, 2011.

237. Nissen SE, Yock P. Intravascular ultrasound: novel pathophysiological insights and current clinical applications. Circulation. 2001;103(4): 604-616.

238. Nicholls SJ, Hsu A, Wolski K, et al. Intravascular ultrasound-derived measures of coronary atherosclerotic plaque burden and clinical outcome. J Am Coll Cardiol. 2010;55(21):2399-2407.

239. DeMaria AN, Narula J, Mahmud E, Tsimikas S. Imaging vulnerable plaque by ultrasound. J Am Coll Cardiol. 2006;47(Suppl 8):C32-C39.

240. Maehara A, Mintz GS, Cristea E, et al. Even after percutaneous coronary intervention of angiographically significant lesions, IVUS-defined high-grade stenoses are common. A baseline IVUS analysis from The Prospect Trial. J Am Coll Cardiol. 2010;55: A204.E1921.

241. Briguori C, Tobis J, Nishida T, et al. Discrepancy between angiography and intravascular ultrasound when analysing small coronary arteries. Eur Heart J. 2002;23(3):247-254.

242. Ballantyne CM, Raichlen JS, Nicholls S, et al; ASTEROID Investigators. Effect of rosuvastatin therapy on coronary artery stenoses assessed by quantitative coronary angiography: a study to evaluate the effect of rosuvastatin on intravascular ultrasound-derived coronary atheroma burden. Circulation. 2008;117(19):2458-2466.

243. Bezerra HG, Costa MA, Guagliumi G, Rollins AM, and Simon DI. Intracoronary optical coherence tomography: a comprehensive review: clinical and research applications. JACC Cardiovasc Interv. 2009; 2(11):1035-1046.

244. Kubo T, Imanishi T, Kashiwagi M, et al. Multiple coronary lesion instability in patients with acute myocardial infarction as determined by optical coherence tomography. Am J Cardiol. 2010;105(3): 318-322.

245. Yamamoto T, Lu C, Ryan RO. A Two-step binding model of PCSK9 Interaction with the low density lipoprotein receptor. $J$ Biol Chem. 2011;286(7):5464-5470.

246. Horton JD, Cohen JC, Hobbs HH. PCSK9: a convertase that coordinates LDL catabolism. J Lipid Res. 2009;50 Suppl:S172-S177.

247. Cohen JC, Boerwinkle E, Mosley TH Jr, Hobbs HH. Sequence variations in PCSK9, low LDL, and protection against coronary heart disease. N Engl J Med. 2006;354(12):1264-1272.

248. Benn M, Nordestgaard BG, Grande P, Schnohr P, Tybjærg-Hansen A. PCSK9 R46L, low-density lipoprotein cholesterol levels, and risk of ischemic heart disease: 3 independent studies and meta-analyses. J Am Coll Cardiol. 2010;55(25):2833-2842.

249. Abifadel M, Varret M, Rabes JP, et al. Mutations in PCSK9 cause autosomal dominant hypercholesterolemia. Nat Genet. 2003;34(2): $154-156$. 
250. Myocardial Infarction Genetics Consortium Proprotein convertase subtilisin/kexin type 9 (PCSK9) missense variant is reproducibly associated with early-onset myocardial infarction in $>1500$ cases and 1500 controls (abstr). Circulation. 2007;116:II806.

251. Steinberg D. Earlier intervention in the management of hypercholesterolemia: what are we waiting for? $\mathrm{J} \mathrm{Am} \mathrm{Coll} \mathrm{Cardiol}$. 2010;56(8):627-629.

252. Kones R. Recent advances in the management of chronic stable angina. II. Anti-ischemic therapy, options for refractory angina, risk factor reduction, and revascularization. Vasc Health Risk Manag. 2010;6:749-774.

253. Domanski MJ. Primary prevention of coronary artery disease. $N$ Engl J Med. 2007;357(15):1543-1545.

254. Redberg R, Benjamin EJ, Bittner V, et al. ACCF/AHA 2009 Performance measures for primary prevention of cardiovascular disease in adults: a report of the American College of Cardiology Foundation/American Heart Association Task Force on Performance Measures (Writing Committee to Develop Performance Measures for Primary Prevention of Cardiovascular Disease) developed in collaboration with the American Academy of Family Physicians; American Association of Cardiovascular and Pulmonary Rehabilitation; and Preventive Cardiovascular Nurses Association: endorsed by the American College of Preventive Medicine, American College of Sports Medicine, and Society for Women's Health Research. J Am Coll Cardiol. 2009;54(14):1364-1405.

255. Forrester JS. Redefining normal low-density lipoprotein cholesterol: a strategy to unseat coronary disease as the nation's leading killer. J Am Coll Cardiol. 2010;56(8):630-636.

256. DeMazumder D, Hasan RK, Blumenthal RS, Michos ED, Jones S. Should statin therapy be allocated on the basis of global risk or on the basis of randomized trial evidence? Am J Cardiol. 2010;106(6):905-909.

257. Nambi V, Ballantyne CM. Utility of statin therapy using high-sensitivity C-reactive protein as an indicator of coronary heart disease risk. Curr Atheroscler Rep. 2005;7(1):22-28.

258. Ridker PM. High-sensitivity C-reactive protein and cardiovascular risk: rationale for screening and primary prevention. Am J Cardiol. 2003;92(4B):17K-22K.

259. Mähönen MS, McElduff P, Dobson AJ, Kuulasmaa KA, Evans AE; for the WHO MONICA Project. Current smoking and the risk of non-fatal myocardial infarction in the WHO MONICA Project populations. Tob Control. 2004;13(3):244-250.

260. Bakhru A, Erlinger TP. Smoking cessation and cardiovasacular disease risk factors: Results from the third National Health and Nutrition Examination Survey. PLoS Med. 2005;2(6):e160.

261. Yusuf S, Lonn E, Bosch J. Lipid lowering for primary prevention. Lancet. 2009;373(9670):1152-1155.

262. Burt VL, Cutler JA, Higgins M, et al. Trends in the prevalence, awareness, treatment, and control of hypertension in the adult US population. Data from the health examination surveys, 1960 to 1991. Hypertension. 1995;26(1):60-69.

263. Dolan E, Stanton A, Thijs L, et al. Superiority of ambulatory over clinic blood pressure measurement in predicting mortality: the Dublin outcome study. Hypertension. 2005;46(1):156-161.

264. Yeh RW, Sidney S, Chandra M, Sorel M, Selby J, Go AS. Population trends in the incidence and outcomes of acute myocardial infarction. N Engl J Med. 2010;362(23):2155-2165.

265. Brown JR, O'Connor GT. Coronary heart disease and prevention in the United States. $N$ Eng J Med. 2010;362(23): 2150-2153.

266. Garcia-Garcia HM, Costa MA, Serruys PW. Imaging of coronary atherosclerosis: intravascular ultrasound. Eur Heart J. 2010;31(20): 2456-2469.

267. Sipahi I, Nicholls SJ, Tuzcu EM, Nissen SE. Coronary atherosclerosis can regress with very intensive statin therapy. Cleve Clin J Med. 2006; 73(10):937-944.

268. Mack WJ, Xiang M, Selzer RH, Hodis HN. Serial quantitative coronary angiography and coronary events. Am Heart J. 2000;139(6): 993-999.
269. Buchwald H, Matts JP, Fitch LL, et al; for the Program on the Surgical Control of the Hyperlipidemias (POSCH) Group. Changes in sequential coronary arteriograms and subsequent coronary events. JAMA. 1992;268(11):1429-1433.

270. Petronio AS, Amoroso G, Limbruno U, et al. Simvastatin does not inhibit intimal hyperplasia and restenosis but promotes plaque regression in normocholesterolemic patients undergoing coronary stenting: a randomized study with intravascular ultrasound. $A m$ Heart J. 2005;149(3):520-526.

271. Tani S, Watanabe I, Anazawa T, et al. Effect of pravastatin on malondialdehyde-modified low-density lipoprotein levels and coronary plaque regression as determined by threedimensional intravascular ultrasound. Am J Cardiol. 2005;96(8): 1089-1094.

272. Takashima H, Ozaki Y, Yasukawa T, et al. Impact of lipid-lowering therapy with pitavastatin, a new HMG-CoA reductase inhibitor, on regression of coronary atherosclerotic plaque. Circ J. 2007;71(11): $1678-1684$.

273. Takayama T, Hiro T, Yamagishi M, et al. Effect of rosuvastatin on coronary atheroma in stable coronary artery disease: multicenter coronary atherosclerosis study measuring effects of rosuvastatin using intravascular ultrasound in Japanese subjects (COSMOS). Circ J. 2009;73(11):2110-2117.

274. Hiro T, Kimura T, Morimoto T, et al. Effect of intensive statin therapy on regression of coronary atherosclerosis in patients with acute coronary syndrome: a multicenter randomized trial evaluated by volumetric intravascular ultrasound using pitavastatin versus atorvastatin (JAPAN-ACS [Japan assessment of pitavastatin and atorvastatin in acute coronary syndrome] study). J Am Coll Cardiol. 2009;54(4):293-302.

275. Hirayama A, Saito S, Ueda Y, et al. Qualitative and quantitative changes in coronary plaque associated with atorvastatin therapy. Circ J. 2009;73(4):718-725.

276. Inoue K, Motoyama S, Sarai M, et al. Serial coronary CT angiographyverified changes in plaque characteristics as an end point: evaluation of effect of statin intervention. JACC Cardiovasc Imaging. 2010;3(7): 691-698.

277. Stone GW, Maehara A, Lansky AJ, et al; for the PROSPECT Investigators. A prospective natural-history study of coronary atherosclerosis. N Engl J Med. 2011;364(3):226-235.

278. Bayturan O, Kapadia S, Nicholls SJ, et al. Clinical predictors of plaque progression despite very low levels of low-density lipoprotein cholesterol. J Am Coll Cardiol. 2010;55(24):2736-2742.

279. Ridker PM, Danielson E, Fonseca FA, et al. Reduction in C-reactive protein and LDL cholesterol and cardiovascular event rates after initiation of rosuvastatin: a prospective study of the JUPITER trial. Lancet. 2009;373(9670):1175-1182.

280. Van der Zijl NJ, Muskiet HA, Tushuizen ME, et al. C-reactive protein mediates the association of liver fat and carotid intima-media thickness in healthy men and men with the metabolic syndrome and/or uncomplicated type 2 diabetes. Diab Met Syndr Clin Res Rev. 2010;4(3): $160-164$.

281. JBS 2: Joint British Societies' guidelines on prevention of cardiovascular disease in clinical practice. Heart. 2005;91(Suppl 5): v1-v52.

282. Chobanian AV, Bakris GL, Black HR, et al. Seventh report of the Joint National Committee on Prevention, Detection, Evaluation, and Treatment of High Blood Pressure. Hypertension. 2003;42(6): 1206-1252.

283. NHS National Institute for Health and Clinical Excellence. Statins for the prevention of cardiovascular events. Technology appraisal 94, January 25, 2006. Available at http://guidance.nice.org.uk/TA94/ Guidance/pdf/English. Accessed on March 24, 2011.

284. NHS National Institute for Health and Clinical Excellence. Prevention of Cardiovascular Disease at Population Level. NICE public health guidance 25. June, 2010. Available at: http://www.nice.org. uk/nicemedia/live/13024/49273/49273.pdf. Accessed March 24, 2011 
285. Royal College of General Practitioners. Lipid modification: cardiovascular risk assessment and the modification of blood lipids for the primary and secondary prevention of cardiovascular disease. NICE Clinical guideline 67. London: RCGP; 2008. Reissued March, 2010. Available at: http://www.nice.org.uk/guidance/index. jsp?action=byID\&o=11982. Accessed March 24, 2011.

286. Grundy SM, Cleeman JI, Merz CN, et al; The Coordinating Committee of the National Cholesterol Education Program. Implications of recent clinical trials for the National Cholesterol Education Program Adult Treatment Panel III Guidelines. Circulation. 2004;110(2): 227-239.

287. Beri A, Contractor T, Khasnis A, Thakur R. Statins and the reduction of sudden cardiac death: antiarrhythmic or anti-ischemic effect? Am J Cardiovasc Drugs. 2010;10(3):155-164.

288. Ward S, Lloyd Jones M, Pandor A, et al. A systematic review and economic evaluation of statins for the prevention of coronary events. Health Technol Assess. 2007;11(14):1-160.

289. Thavendiranathan P, Bagai A, Brookhart MA, Choudhry NK. Primary prevention of cardiovascular diseases with statin therapy: a meta-analysis of randomized controlled trials. Arch Intern Med. 2006;166(21):2307-2313.

290. Mills EJ, Rachlis B, Wu P, Devereaux PJ, Arora P, Perri D. Primary prevention of cardiovascular mortality and events with statin treatments: a network meta-analysis involving more than 65,000 patients. J Am Coll Cardiol. 2008;52(22):1769-1781.

291. Brugts JJ, Yetgin T, Hoeks SE, et al. The benefits of statins in people without established cardiovascular disease but with cardiovascular risk factors: meta-analysis of randomised controlled trials. $B M J$. 2009;338:b2376.

292. Ray KK, Seshasai SRK, Erqou S; et al. Statins and all-cause mortality in high-risk primary prevention: a meta-analysis of 11 randomized controlled trials involving 65,229 participants. Arch Intern Med. 2010; 170(12):1024-1031.

293. Pasternak RC, Smith SC Jr, Bairey-Merz CN, Grundy SM, Cleeman JI, Lenfant C. ACC/AHA/NHLBI Clinical advisory on the use and safety of statins. J Am Coll Cardiol. 2002;40(3):567-572.

294. Grundy SM, Cleeman JI, Merz CN, et al; National Heart, Lung, and Blood Institute; American College of Cardiology Foundation; American Heart Association. Implications of recent clinical trials for the National Cholesterol Education Program Adult Treatment Panel III guidelines. Circulation. 2004;110(2):227-239.

295. Bukkapatnam RN, Gabler NB, Lewis WR. Statins for primary prevention of cardiovascular mortality in women: a systematic review and meta-analysis. Prev Cardiol. 2010;13(2):84-90.

296. Nakamura H, Arakawa K, Itakura H, et al; MEGA Study Group. Primary prevention of cardiovascular disease with pravastatin in Japan (MEGA Study): a prospective randomized controlled trial. Lancet. 2006;368(9542):1155-1163

297. Nakamura H. Primary prevention of cardiovascular diseases among hypercholesterolemic Japanese with a low dose of pravastatin. Atheroscler Suppl. 2007;8(2):13-17.

298. Taylor F, Ward K, Moore THM, et al. Statins for the primary prevention of cardiovascular disease. Cochrane Database Syst Rev. 2011;1:CD004816.

299. Gotto AM. Improving lipid goal attainment. is it enough? Circulation. 2009;120(1):3-5.

300. Pletcher MJ, Hulley SB. Statin therapy in young adults: ready for prime time? J Am Coll Cardiol. 2010;56(8):637-640.

301. Cooper A, O'Flynn N. Risk assessment and lipid modification for primary and secondary prevention of cardiovascular disease: summary of NICE guidance. BMJ. 2008;336(7655):1246-1248.

302. Lee KK, Cipriano LE, Owens DK, GoAS, Hlatky MA. Cost-effectiveness of using high-sensitivity C-reactive protein to identify intermediateand low-cardiovascular-risk individuals for statin therapy. Circulation. 2010;122(15):1478-1487.

303. Vaklavas C, Chatzizisis YS, Ziakas A, Zamboulis C, Giannoglou GD. Molecular basis of statin-associated myopathy. Atherosclerosis. 2009;202(1):18-28.
304. Mas E, Mori TA. Coenzyme Q10 and statin myalgia: what is the evidence? Curr Atheroscler Rep. 2010;12(6);407-413.

305. Vandenberg BF, Robinson J. Management of the patient with statin intolerance. Curr Atheroscler Rep. 2010;12(1):48-57.

306. Thompson PD, Clarkson PM, Rosenson RS, National Lipid Association Statin Safety Task Force Muscle Safety Expert Panel. An assessment of statin safety by muscle experts. Am J Cardiol. 2006; 97(8A):69C-76C

307. Deedwania P, Stone PH, Bairey Merz CN, et al. Effects of intensive versus moderate lipid-lowering therapy on myocardial ischemia in older patients with coronary heart disease: results of the Study Assessing Goals in the Elderly (SAGE). Circulation. 2007;115(6):700-707.

308. Gotto AM Jr. Statin therapy and the elderly: SAGE Advice? Circulation. 2007;115(6):681-683.

309. Golomb B. Statin adverse effects: implications for the elderly. Geriatric Times. 2004;5(4):18-20.

310. Nichols GA, Nag S, Chan W. Intensity of lipid-lowering therapy and low-density lipoprotein cholesterol goal attainment among the elderly before and after the 2004 National Cholesterol Education Program Adult Treatment Panel III update. Am Heart J. 2007;154;(3):554-560.

311. Maroo BP, Lavie CJ, Milani RV Efficacy and safety of intensive statin therapy in the elderly. Am J Geriatr Cardiol. 2008;17(2):92-100.

312. Wenger NK, Lewis SJ. Use of Statin Therapy to Reduce Cardiovascular Risk in Older Patients. Curr Gerontol Geriatr Res. 2010:915296.

313. Long SB, Blaha MJ, Blumenthal RS, Michos ED. Clinical utility of rosuvastatin and other statins for cardiovascular risk reduction among the elderly. Clin Interv Aging. 2011;6:27-35.

314. Pletcher MJ, Coxson PG. Does age modify effectiveness of statin therapy in coronary artery disease? Ann Intern Med. 2010; 152(12):825.

315. Lewington S, Whitlock G, Clarke R, et al; Prospective Studies Collaboration. Blood cholesterol and vascular mortality by age, sex, and blood pressure: a meta-analysis of individual data from 61 prospective studies with 55,000 vascular deaths. Lancet. 2007; 370(9602):1829-1839.

316. Pletcher MJ, Lazar L, Bibbins-Domingo K, et al. Comparing impact and cost effectiveness of primary prevention strategies for lipidlowering. Ann Intern Med. 2009;150(4):243-254.

317. Hayward RA. Does age modify effectiveness of statin therapy in coronary artery disease? Ann Intern Med. 2010;152(12):825-826.

318. Glynn RJ, Koenig W, Nordestgaard BG, Shepherd J, Ridker PM. Rosuvastatin for primary prevention in older persons with elevated $\mathrm{C}$-reactive protein and low to average low-density lipoprotein cholesterol levels: exploratory analysis of a randomized trial. Ann Intern Med. 2010;152(8):488-496.

319. Zieman SJ, Ouyang P. Statins for primary prevention in older adults: who is high risk, who is old, and what denotes primary prevention? Ann Int Med. 2010;152(8):528-530.

320. Jaffer FA, O'Donnell CJ, Larson MG, et al. Age and sex distribution of subclinical aortic atherosclerosis: a magnetic resonance imaging examination of the Framingham Heart Study. Arterioscler Thromb Vasc Biol. 2002;22(5):849-854.

321. Cannon $\mathrm{CP}, \mathrm{Braunwald} \mathrm{E}, \mathrm{McCabe} \mathrm{CH}$, et al. Intensive versus moderate lipid lowering with statins after acute coronary syndromes. $N$ Engl $J$ Med. 2004;350(15):1495-1504.

322. Ray KK, Cannon, Cairns R, et al; PROVE IT-TIMI 22 Investigators. Relationship between uncontrolled risk factors and C-reactive protein levels in patients receiving standard or intensive statin therapy for acute coronary syndromes in the PROVE IT-TIMI 22 Trial. J Am Coll Cardiol. 2005;46(8):1417-1424.

323. Miller M, Cannon CP, Murphy SA, et al. Impact of triglyceride levels beyond low-density lipoprotein cholesterol after acute coronary syndrome in the PROVE-IT TIMI 22 trial. J Am Coll Cardiol. 2008; 51(7):724-730.

324. Sacks FM, Tonkin AM, Craven T, et al. Coronary heart disease in patients with low LDL-cholesterol: benefit of pravastatin in diabetics and enhanced role for HDL-cholesterol and triglycerides as risk factors. Circulation. 2002;105(12):1424-1428. 
325. Barter P, Gotto AM, LaRosa JC, et al. HDL cholesterol, very low levels of LDL cholesterol, and cardiovascular events. $N$ Engl J Med. 2007;357(13):1301-1310.

326. Arsenault BJ, Rana JS, Stroes ESG, et al. Beyond low-density lipoprotein cholesterol: respective contributions of non- high density lipoprotein cholesterol levels, triglycerides, and the total cholesterol/ high-density lipoprotein cholesterol ratio to coronary heart disease risk in apparently healthy men and women. J Am Coll Cardiol. 2010;55(1): 35-41.

327. Carey VJ, Bishop L, Laranjo N, Harshfield BJ, Kwiat C, Sacks FM. Contribution of high plasma triglycerides and low high-density lipoprotein cholesterol to residual risk of coronary heart disease after establishment of low-density lipoprotein cholesterol control. Am J Cardiol. 2010;106(6):757-763.

328. Núñez-Cortés JM, Juan Montoya JP-B, Salas XP, et al. Estudio REALIST (REsiduAl risk, LIpids and Standard Therapies): Un análisis del Riesgo Residual dependiente del perfil lipídico en el síndrome coronario agudo. Endocrinol Nutr. 2011;58(1):38-47.

329. Neil CE, Nicklas TA. Relationship Between Diet/Physical Activity and Health. Am J Lifestyle Med. 2007;1(6):457-481.

330. Stevens J, McClain JE, Truesdale JP. Commentary: Obesity claims and controversies. Int J Epidemiol. 2006;35(1):77-78.

331. Chamberlain J, Francis S, Brookes Z, et al. Interleukin-1 regulates multiple atherogenic mechanisms in response to fat feeding. PLoS ONE. 2009;4(4):e5073.

332. Lamon BD, Hajjar DP. Inflammation at the molecular interface of atherogenesis: an anthropological journey. Am J Pathol. 2008;173(5):1253-1264.

333. McLaughlin T, Abbasi F, Lamendola C, Reaven G. Heterogeneity in the prevalence of risk factors for cardiovascular disease and type 2 diabetes mellitus in obese individuals: effect of differences in insulin sensitivity. Arch Intern Med. 2007;167(7):642-648.

334. Goldstein BJ, Scalia R. Adiponectin: a novel adipokine linking adipocytes and vascular function. J Clin Endocrinol Metab. 2004;89(6) 2563-2568.

335. Bray GA. Medical consequences of obesity. J Clin Endocrinol Metab. 2004;89(6):2583-2589.

336. Kershaw EE, Flier JS. Adipose tissue as an endocrine organ. J Clin Endocrinol Metab. 2004;89(6):2548-2556.

337. Ziemke F, Mantzoros CS. Adiponectin in insulin resistance: lessons from translational research. Am J Clin Nutr. 2010;91(1):258S-261S.

338. Hajer GR, van Haeften TW, Visseren FLJ. Adipose tissue dysfunction in obesity, diabetes, and vascular diseases. Eur Heart J. 2008; 29(24):2959-2971.

339. Boden G. Endoplasmic reticulum stress: another link between obesity and insulin resistance/inflammation? Diabetes. 2009;58(3):518-519.

340. Rasouli N and Kern PA. Adipocytokines and the Metabolic Complications of Obesity. J Clin Endocrinol Metab. 2008;93(11 Supp1):s64-s73.

341. Zhang H, Zhang C. Adipose "talks' to distant organs to regulate insulin sensitivity and vascular function. Obesity. 2010;18(11):2071-2076.

342. Berman DS, Cheng VY, Dey D. Not all body fat weighs equally in the acceleration of coronary artery disease. J Am Coll Cardiol Img. 2010;3(9)918-920.

343. Ohashi N, Yamamoto H, Horiguchi J, et al. Association between visceral adipose tissue area and coronary plaque morphology assessed by ct angiography. JACC Cardiovasc Imaging. 2010;3(9): 908-917.

344. Hsia J, MacFadyen JG, Monyak J, Ridker PM. Cardiovascular event reduction and adverse events among subjects attaining low-density lipoprotein cholesterol $50 \mathrm{mg} / \mathrm{dl}$ with rosuvastatin. J Am Coll Cardiol. 2011;57(16):1666-1675.

345. McMurray JJ, Holman RR, Haffner SM, et al; The NAVIGATOR Study Group. Effect of valsartan on the incidence of diabetes and cardiovascular events. N Engl J Med. 2010;362(16):1477-1490.

346. Holman RR, Haffner SM, McMurray JJ, et al; The NAVIGATOR Study Group. Effect of nateglinide on the incidence of diabetes and cardiovascular events. $N$ Engl J Med. 2010;362(16): $1463-1476$.
347. Ginsberg HN, Elam MB, Lovato LC, et al; for The ACCORD Study Group. Effects of Combination Lipid Therapy in Type 2 Diabetes Mellitus. N Engl J Med. 2010;362(17):1563-1574.

348. Nilsson PM. ACCORD and Risk-Factor Control in Type 2 Diabetes. N Eng J Med. 2010;362(17):1628-1630.

349. Nicholls SJ, Lundman P, Tardif J-C. Diabetic dyslipidemia: extending the target beyond LDL cholesterol. Eur J Cardiovasc Prev Rehabil. 2010;17(suppl 1):s20-s24.

350. Hermans MP, Fruchaart J-C. Reducing residual vascular risk in patients with atherogenic dyslipidemia: Where do we go from here? Clin Lipidol. 2010;5(6):811-826.

351. Gordon DJ, Probstfield JL, Garrison RJ, et al. High-density lipoprotein cholesterol and cardiovascular disease. Four prospective American studies. Circulation. 1989;79(1):8-15.

352. Nicholls SJ, Tuzcu EM, Sipaghi I, et al. Statins, high-density lipoprotein cholesterol, and regression of coronary atherosclerosis. JAMA. 2007;297(5):499-508.

353. Brown B, Zhao X, Cheung M. Should both HDL-C and LDL-C be targets for lipid therapy? A review of current evidence. J Clin Lipidol. 2007;1(1):88-94.

354. Ghandehari H, Kamal-Bahl S, Wong ND. Prevalence and extent of dyslipidemia and recommended lipid levels in US adults with and without cardiovascular comorbidities: the National Health and Nutrition Examination Survey 2003-2004. Am Heart J. 2008;156(1): 112-119.

355. Sachdeva A, Cannon CP, Deedwania PC, et al. Lipid levels in patients hospitalized with coronary artery disease: an analysis of 136,905 hospitalizations in Get With The Guidelines. Am Heart J. 2009; 157(1):111-117.e2.

356. Sharrett AR, Ballantyne CM, Coady SA, et al. Coronary heart disease prediction from lipoprotein cholesterol levels, triglycerides, lipoprotein(a), apolipoproteins A-I and B, and HDL density subfractions: the Atherosclerosis Risk in Communities (ARIC) Study. Circulation. 2001;104(10):1108-1113.

357. Emerging Risk Factors Collaboration. Major lipids, apolipoproteins, and risk of vascular disease. JAMA. 2009;302(18):1993-2000.

358. Briel M, Ferreira-Gonzalez I, You JJ, et al. Association between change in high density lipoprotein cholesterol and cardiovascular disease morbidity and mortality: systematic review and meta-regression analysis. BMJ. 2009;338:b92.

359. Gotto AM, Whitney E, Stein EA, et al. Relation between baseline and on-treatment lipid parameters and first acute major coronary events in the Air Force/Texas Coronary Atherosclerosis Prevention Study (AFCAPS/TexCAPS). Circulation. 2000;101(5):477-484

360. Jones PH, Davidson MH, Stein EA, et al. Comparison of the efficacy and safety of rosuvastatin versus atorvastatin, simvastatin, and pravastatin across doses (STELLAR* Trial). Am J Cardiol. 2003; 92(2):152-160.

361. Jones PH, Hunninghake DB, Ferdinand KC, et al. Effects of rosuva-statin versus atorvastatin, simvastatin, and pravastatin on non-high-density lipoprotein cholesterol, apolipoproteins, and lipid ratios in patients with hypercholesterolemia: additional results from the STELLAR trial. Clin Ther. 2004;26(9):1388-1399.

362. Negi S, Ballantyne CM. Insights from recent meta-analysis: role of high-density lipoprotein cholesterol in reducing cardiovascular events and rates of atherosclerotic disease progression. J Clin Lipidol. 2010;4(5):365-370.

363. CRESTOR Athero Imaging Head to Head IVUS Study (SATURN). National Institutes of Health. 25 January 2010; Available at: http:// clinicaltrials.gov/ct2/show/NCT00620542. Accessed March 24, 2011.

364. Saleem TSM, Rani PVS, Gauthaman K. High-density lipoproteins: a novel therapeutic target for cardiovascular disease. Res Reports Clin Cardiol. 2011;2:1-6.

365. Vaisar T, Pennathur S, Green PS, et al. Shotgun proteomics implicates protease inhibition and complement activation in the antiinflammatory properties of HDL. J Clin Invest. 2007;117(3): 746-756. 
366. Greene DJ, Skeggs JW, Morton RE. Elevated triglyceride content diminishes the capacity of high density lipoprotein to deliver cholesteryl esters via the scavenger receptor class B type I (SR-BI). J Biol Chem. 2001;276(7):4804-4811.

367. Saddar S, Mineo C, Shaul PW. Signaling by the high-affinity hdl receptor scavenger receptor B type I. Arterioscler Thromb Vasc Biol. 2010;30(2):144-150

368. Navab M, Reddy ST, Van Lenten BJ, Fogelman AM. HDL and cardiovascular disease: atherogenic and atheroprotective mechanisms. Nat Rev Cardiol. 2011;8(4):222-232.

369. Brewer HB Jr, Santamarina-Fojo S. New insights into the role of the adenosine triphosphate-binding cassette transporters in high-density lipoprotein metabolism and reverse cholesterol transport. Am J Cardiol. 2003;91(7A):3E-11E

370. Navab M, Shechter I, Anantharamaiah GM, Reddy ST, Van Lenten BJ, Fogelman AM. structure and function of HDL mimetics. Arterioscler Thromb Vasc Biol. 2010;30(2):164-168.

371. Masson D, Jiang XC, Lagrost L, Tall AR. The role of plasma lipid transfer proteins in lipoprotein metabolism and atherogenesis. J Lipid Res. 2009;50(Suppl):S201-S206.

372. Khera AV, Cuchel M. Margarita de la Llera-Moya, et al. Cholesterol efflux capacity, high-density lipoprotein function, and atherosclerosis. N Engl J Med. 2011;364(2):127-135.

373. Heinecke J. HDL and Cardiovascular-Disease risk - time for a new approach? N Engl J Med. 2011;364(2):170-171.

374. Rosenblat M, Aviram M. Paraoxonases role in the prevention of cardiovascular diseases. Biofactors. 2009;35(1):98-104.

375. Getz GS, Reardon CA. Paraoxonase, a cardioprotective enzyme: continuing issues. Curr Opin Lipidol. 2004;15(3):261-267.

376. Andersen RV, Wittrup HH, Tybjaerg-Hansen A, Steffensen R, Schnohr P, Nordestgaard BG. Hepatic lipase mutations, elevated high-density lipoprotein cholesterol, and increased risk of ischemic heart disease: the Copenhagen City Heart Study. J Am Coll Cardiol. 2003;41(11):1972-1982.

377. Corsetti JP, Zareba W, Moss AJ, Rainwater DL, Sparks CE. Elevated HDL is a risk factor for recurrent coronary events in a subgroup of non-diabetic postinfarction patients with hypercholesterolemia and inflammation. Atherosclerosis. 2006;187(1): 191-197.

378. DeGoma EM, Leeper NJ, Heidenreich PA. Clinical significance of high-density lipoprotein cholesterol in patients with low lowdensity lipoprotein cholesterol. J Am Coll Cardiol. 2008;51(1): 49-55.

379. van der Steeg WA, Holme I, Boekholdt SM, et al. High-density lipoprotein cholesterol, high-density lipoprotein particle size, and apolipoprotein A-I: significance for cardiovascular risk. $J$ Am Coll Cardiol. 2008;51(6):634-642.

380. Corsetti JP, Gansevoort RT, Sparks CE, Dullaart RPF. HDL inflammation reduces HDL protection against primary cardiac risk. Eur J Clin Invest. 2010;40(6):483-489.

381. Dullaart RPF. Increased coronary heart disease risk determined by high high-density lipoprotein cholesterol and c-reactive protein: modulation by variation in the CETP gene. Arterioscler Thromb Vasc Biol. 2010;30(8):1502-1503.

382. Rossouw JE, Anderson GL, Prentice RL, et al; Writing Group for the Women's Health Initiative Investigators. Risks and benefits of estrogen plus progestin in healthy postmenopausal women: principal results from the Women's Health Initiative randomized controlled trial. JAMA. 2002;288(3):321-333.

383. Davidson WS, Silva RAGD, Chantepie S Lagor WR, Chapman MJ, Kontush A. Proteomic analysis of defined HDL subpopulations reveals particle-specific protein clusters: relevance to antioxidative function. Arterioscler Thromb Vasc Biol. 2009;29(6):870-876.

384. Yancey PG, Bortnick AE, Kellner-Weibel G, et al. Importance of different pathways of cellular cholesterol efflux. Arterioscler Thromb Vasc Biol. 2003;123(5):712-719.
385. Kontush A, Chapman MJ. Antiatherogenic small, dense HDL-guardian angel of the arterial wall? Nat Clin Pract Cardiovasc Med. 2006; 3(3):144-153.

386. Zheng L, Nukuna B, Brennan M-L, et al. Apolipoprotein A-I is a selective target for myeloperoxidase-catalyzed oxidation and functional impairment in subjects with cardiovascular disease. J Clin Invest. 2004;114(4):529-541.

387. Sacks FM, Alaupovic P, Moye LA, et al. VLDL, apolipoproteins B, CIII, and $\mathrm{E}$, and risk of recurrent coronary events in the Cholesterol and Recurrent Events (CARE) trial. Circulation. 2000;102(16): 1886-1892.

388. Shao B, Oda MN, Oram JF, Heinecke JW. Myeloperoxidase: an oxidative pathway for generating dysfunctional high-density lipoprotein. Chem Res Toxicol. 2010;23(3):447-454.

389. Norata GD, Pirillo A, Catapano AL. Modified HDL: biological and physiopathological consequences. Nutr Metabol Cardiovasc Dis. 2006;16(5):371-386.

390. Khovidhunkit W, Kim MS, Memon RA, et al. Effects of infection and inflammation on lipid and lipoprotein metabolism: mechanisms and consequences to the host. J Lipid Res. 2004;45(7):1169-1196.

391. Esteve E, Ricart W, Fernandez-Real JM. Dyslipidemia and inflammation: an evolutionary conserved mechanism. Clin Nutr. 2005; 24(1):16-31

392. Navab M, Anantharamaiah GM, Reddy ST, et al. The oxidation hypothesis of atherogenesis: the role of oxidized phospholipids and HDL. J Lipid Res. 2004;45(6):993-1007.

393. Ansell BJ, Navab M, Hama S, et al. Inflammatory/antiinflammatory properties of high-density lipoprotein distinguish patients from control subjects better than high-density lipoprotein cholesterol levels and are favorably affected by simvastatin treatment. Circulation. 2003; 108(22):2751-2756.

394. Briffa TG, Hobbs MS, Tonkin A, et al. Population trends of recurrent coronary heart disease event rates remain high. Circ Cardiovasc Qual Outcomes. 2010;4(1):107-113.

395. Tall AR, Breslow JL, Rubin EM. Genetic disorders affecting plasma high-density lipoproteins. Available at: http://www.ommbid.com/ OMMBID/the_online_metabolic_and_molecular_bases_of_inherited_ disease/b/abstract/part12/ch121. Accessed March 24, 2011.

396. Ansell BJ. Targeting the anti-inflammatory effects of high-density lipoprotein. Am J Cardiol. 2007;100(11A):n3-9.

397. Green PS, Vaisar T, Pennathur S, et al. Combined statin and niacin therapy remodels the high-density lipoprotein proteome. Circulation. 2008;118(12):1259-1267.

398. Sorrentino SA, Besler C, Rohrer L, et al. Endothelial-vasoprotective effects of high-density lipoprotein are impaired in patients with type 2 diabetes mellitus but are improved after extended-release niacin therapy. Circulation. 2010;121(1):110-122.

399. Heslop CL, Frohlich JJ, Hill JS. Myeloperoxidase and C-reactive protein have combined utility for long-term prediction of cardiovascular mortality after coronary angiography. J Am Coll Cardiol. 2010;55(11): 1102-1109.

400. Singh U, Devaraj S, Jialal I. C-reactive protein stimulates myeloperoxidase release from polymorphonuclear cells and monocytes: implications for acute coronary syndromes. Clin Chem. 2009;55(2):361-364.

401. Rashid S, Watanabe T, Sakaue T, Lewis GF. Mechanisms of HDL lowering in insulin resistant, hypertriglyceridemic states: the combined effect of HDL triglyceride enrichment and elevated hepatic lipase activity. Clin Biochem. 2003;36(6):421-429.

402. Xiao C, Watanabe T, Zhang Y, et al. Enhanced cellular uptake of remnant high-density lipoprotein particles: a mechanism for highdensity lipoprotein lowering in insulin resistance and hypertriglyceridemia. Circ Res. 2008;103(2):159-166.

403. Brown RJ, Rader DJ. When HDL Gets Fat. Circ Res. 2008;103(2): $131-132$.

404. Hansel B, Giral P, Nobecourt E, et al. Metabolic syndrome is associated with elevated oxidative stress and dysfunctional dense high-density lipoprotein particles displaying impaired antioxidative activity. J Clin Endocrinol Metab. 2004;89(10):4963-4971. 
405. Nobecourt E, Jacqueminet S, Hansel B, et al. Defective antioxidative activity of small, dense HDL particles in type 2 diabetes: relationship to elevated oxidative stress and hyperglycemia. Diabetologia. 2005; 48(3):529-38.

406. Kontush A, Chapman MJ. Functionally defective high-density lipoprotein: a new therapeutic target at the crossroads of dyslipidemia, inflammation, and atherosclerosis. Pharmacol Rev. 2006;58(3): 342-374.

407. Getz GS, Reardon CA. High-density lipoprotein function in regulating insulin secretion: possible relevance to metabolic syndrome. Arterioscler Thromb Vasc Biol. 2010;30(8):1497-1499.

408. Oksjoki R, Kovanen PT, Pentikainen MO. Role of complement activation in atherosclerosis. Curr Opin Lipidol. 2003;14(5):477-482.

409. Heinecke JW. The protein cargo of HDL: Implications for vascular wall biology and therapeutics. J Clin Lipidol. 2010;4(5):371-375.

410. McGillicuddy FC, de la Llera MM, Hinkle CC, et al. Inflammation impairs reverse cholesterol transport in vivo. Circulation. 2009; 119(8):1135-1145.

411. Khovidhunkit W, Moser AH, Shigenaga JK, Grunfeld C, Feingold KR. Endotoxin down-regulates ABCG5 and ABCG8 in mouse liver and ABCA1 and ABCG1 in J774 murine macrophages: differential role of LXR. J Lipid Res. 2003;44(9):1728-1736.

412. Annema W, Nijstad N, Tolle M, et al. Myeloperoxidase and serum amyloid A contribute to impaired in vivo reverse cholesterol transport during the acute phase response but not group IIA secretory phospholipase A(2). J Lipid Res. 2010;51(4):743-754.

413. Inouye $M$, Silander $\mathrm{K}$, Hamalainen $\mathrm{E}$, et al. An immune response network associated with blood lipid levels. PLoS Genet. 2010;6(9): e1001113.

414. Weissglas-Volkov D, Pajukanta P. Genetic causes of high and low serum HDL-cholesterol. J Lipid Res. 2010;51(8):2032-2057.

415. Corsetti JP, Ryan D, Rainwater DL, Moss AJ, Zareba W, Sparks CE. Cholesteryl ester transfer protein polymorphism (TaqIB) associates with risk in postinfarction patients with high $\mathrm{C}$-reactive protein and high-density lipoprotein cholesterol levels. Arterioscler Thromb Vasc Biol. 2010;30(8):1657-1664.

416. Navab M, Hama SY, Hough GP, Subbanagounder G, Reddy ST, Fogelman AM. A cell-free assay for detecting HDL that is dysfunctional in preventing the formation of or inactivating oxidized phospholipids. J Lipid Res. 2001;42(8):1308-1317.

417. Karthikeyan G, Teo KK, Islam S, et al. Lipid profile, plastma apolipoproteins, and risk of a first myocardial infarction among Asians: an analysis from the INTERHEART study. J Am Coll Cardiol. 2009; 53(3):244-253.

418. Dodani S, Kaur R, Reddy S, Reed GL, Navab M, George V. Can dysfunctional HDL explain high coronary artery disease risk in south Asians? Int J Cardiol. 2008;129(1):125-132.

419. Dodani S. Can conventional risk factors explain excess coronary artery disease risk in south asians: dyslipidemias and dysfunctional high density lipoprotein (HDL). Open Epidemiol J. 2009; 2:55-62.

420. Das UN. A defect in $\Delta 6$ and $\Delta 5$ desaturases may be a factor in the initiation and progression of insulin resistance, the metabolic syndrome and ischemic heart disease in South Asians. Lipids Health Dis. 2010;9:130

421. Chapman MJ, Le Goff W, Guerin M, Kontush A. Cholesteryl ester transfer protein: at the heart of the action of lipid-modulating therapy with statins, fibrates, niacin, and cholesteryl ester transfer protein inhibitors. Eur Heart J. 2010;31(2):149-164.

422. Rye KA, Bursill CA, Lambert G, Tabet F, Barter PJ. The metabolism and anti-atherogenic properties of HDL. J Lipid Res. 2009; 50(Suppl):S195-S200.

423. Tsompanidi EM, Brinkmeier MS, Fotiadou EH, Giakoumi SM, Kypreos KE. HDL biogenesis and functions: role of HDL quality and quantity in atherosclerosis. Atherosclerosis. 2010;208(1): $3-9$.
424. Natarajan P, Ray KK, Cannon CP. High-density lipoprotein and coronary heart disease: current and future therapies. J Am Coll Cardiol. 2010;55(13):1283-1299.

425. Dodani S, Grice DG, Joshi S. Is HDL function as important as HDL quantity in the coronary artery disease risk assessment? J Clin Lipidol. 2009;3(2):70-77.

426. Alla VM, Manu K, Mooss A. Targeting residual risk: the rationale for the use of non-HDL cholesterol. Southern Med J. 2010;103(5): 434-437

427. Barter PJ, Caulfield M, Eriksson M, et al. Effects of torcetrapib in patients at high risk for coronary events. N Engl J Med. 2007;357(21): 2109-2122.

428. Toth P. Torcetrapib and atherosclerosis: what happened and where do we go from here? Future Lipidol. 2007;2(3):277-284.

429. Nissen SE, Tardif JC, Nicholls SJ, et al. Effect of torcetrapib on the progression of coronary atherosclerosis. NEngl J Med. 2007;356(13): 1304-1316.

430. Cannon CP, Dansky HM, Davidson M, et al. Design of the DEFINE trial: Determining the efficacy and tolerability of CETP INhibition with AnacEtrapib. Am Heart J. 2009;158(4):513-519e3.

431. Cannon CP, Shah S, Dansky HM, et al. Safety of anacetrapib in patients with or at high risk for coronary heart disease. $N$ Eng J Med. 2010;363(25)2406-2415.

432. Krishna R, Bergman AJ, Jin B, et al. Multiple-dose pharmacodynamics and pharmacokinetics of anacetrapib, a potent cholesteryl ester transfer protein (CETP) inhibitor, in healthy subjects. Clin Pharm Therap. 2008;84(6):679-683.

433. Bloomfield D, Carlson GL, Sapre A, et al. Efficacy and safety of the cholesteryl ester transfer protein inhibitor anacetrapib as monotherapy and coadministered with atorvastatin in dyslipidemic patients. Am Heart J. 2009; 157(2):352-360e2.

434. Krishna R, Anderson MS, Bergman AJ, et al. Effect of the cholesteryl ester transfer protein inhibitor, anacetrapib, on lipoproteins in patients with dyslipidaemia and on 24-h ambulatory blood pressure in healthy individuals: two double-blind, randomised placebo-controlled phase I studies. Lancet. 2007;370(9603):1907-1914.

435. Yvan-Charvet L, Kling J, Pagler T, et al. Cholesterol efflux potential and antiinflammatory properties of high-density lipoprotein after treatment with niacin or anacetrapib. Arterioscler Thromb Vasc Biol. 2010;30(7):1430-1438.

436. Nicholls SJ, Gordon A, Johansson J, et al. Efficacy and safety of a novel oral inducer of apolipoprotein a-i synthesis in statin-treated patients with stable coronary artery disease: a randomized controlled trial. J Am Coll Cardiol. 2011;57(9):1111-1119.

437. Davidson MH. Apolipoprotein A-I therapy: promise, challenges, and disappointment. J Am Coll Cardiol. 2011;57(9):1120-1121.

438. Brown BG, Stukovsky KH, Zhao XQ. Simultaneous low-density lipoprotein-C lowering and high-density lipoprotein-C elevation for optimum cardiovascular disease prevention with various drug classes, and their combinations: a meta-analysis of 23 randomized lipid trials. Curr Opin Lipidol. 2006;17(6):631-636.

439. Taylor AJ, Villines TC, Stanek EJ, et al. Extended-release niacin or ezetimibe and carotid intima-media thickness. $N$ Engl J Med. 2009; 361(22):2113-2122.

440. Niacin plus statin to prevent vascular events. Available at: http:// clinicaltrials.gov/ct/show/NCT00120289. Accessed March 24, 2011.

441. Treatment of HDL to reduce the incidence of vascular events (HPS2-THRIVE). Available at: http://clinicaltrials.gov/ct/show/ NCT00461630. Accessed March 24, 2011.

442. Ingelsson $\mathrm{E}, \mathrm{Sch}$ aefer $\mathrm{EJ}$, Contois $\mathrm{JH}$, et al. Clinical utility of different lipid measures for prediction of coronary heart disease in men and women. JAMA. 2007;298(7):776-785.

443. Parish S, Peto R, Palmer A, et al. The joint effects of apolipoprotein B, apolipoprotein A 1, LDL cholesterol, and HDL cholesterol on risk: 3510 cases of acute myocardial infarction and 9805 controls. Eur Heart J. 2009;30(17):2137-2146. 
444. Lewington S, Whitlock G, Clarke, et al; for Prospective Studies Collaboration. Blood cholesterol and vascular mortality by age, sex, and blood pressure: a meta-analysis of individual data from 61 prospective studies with 55000 vascular deaths. Lancet. 2007; 370(6902):1829-1839.

445. Kastelein JJP, van der Steeg WA, Holme I, et al. Lipids, apolipoproteins, and their ratios in relation to cardiovascular events with statin treatment. Circulation. 2008;117(23):3002-3009.

446. Cui Y, Blumenthal RS, Flaws JA, et al. Non-high-density lipoprotein cholesterol level as a predictor of cardiovascular disease mortality. Arch Intern Med. 2001;161(11):1413-1419.

447. Pischon T, Girman CJ, Sacks FM, Rifai N, Stampfer MJ, Rimm EB. Non-high-density lipoprotein cholesterol and apolipoprotein B in the prediction of coronary heart disease in men. Circulation. 2005; 112(22):3375-3383.

448. El Harchaoui K, van der Steeg WA, Stroes ES, et al. Value of lowdensity lipoprotein particle number and size as predictors of coronary artery disease in apparently healthy men and women: the EPICNorfolk Prospective Population Study. J Am Coll Cardiol. 2007;49(5): 547-553.

449. Mora S, Otvos JD, Rifai N, Rosenson RS, Buring JE, Ridker PM. Lipoprotein particle profiles by nuclear magnetic resonance compared with standard lipid and apolipoproteins in predicting incident cardiovascular disease in women. Circulation. 2009;119(7):931-939.

450. Kathiresan S, Otvos JD, Sullivan LM, et al. Increased small low-density lipoprotein particle number. A prominent feature of the metabolic syndrome in the Framingham Heart Study. Circulation. 2006;113(1):20-29.

451. Otvos JD, Collins D, Freedman SD, et al. Low-density lipoprotein and high-density lipoprotein particle subclasses predict coronary events and are favorably changed by gemfibrozil therapy in the Veterans Affairs High-Density Lipoprotein Intervention Trial. Circulation. 2006;113(12):1556-1563.

452. Hsia J, Otvos JD, Rossouw JE, et al; for the Women's Health Initiative Research Group. Lipoprotein particle concentrations may explain the absence of coronary protection in the women's health initiative hormone trials. Arterioscler Thromb Vasc Biol. 2008;28(9): 1666-1671.

453. Adiels M, Borén J, Caslake MJ, et al. Overproduction of VLDL1 driven by hyperglycemia is a dominant feature of diabetic dyslipidemia. Arterioscler Thromb Vasc Biol. 2005;25(8):1697-1703.

454. Brunzell JD, Davidson M, Furberg CD, et al. lipoprotein management in patients with cardiometabolic risk. consensus conference report from the American Diabetes Association and the American College of Cardiology Foundation. J Am Coll Cardiol. 2008;51(15): $1512-1524$.

455. Contois JH, McConnell JP, Sethi AA, et al. Apolipoprotein B and cardiovascular disease risk: position statement from the AACC lipoproteins and vascular diseases division working group on best practices. Clin Chem. 2009;55(3):407-419.

456. Mora S, Szklo M, Otvos JD, et al. LDL particle subclasses, LDL particle size, and carotid atherosclerosis in the Multi-Ethnic Study of Atherosclerosis (MESA). Atherosclerosis. 2007;192(1):211-217.

457. Shimabukuro M, Higa M, Tanaka H, Shimabukuro T, Yamakawa K, Masuzaki H. Distinct effects of pitavastatin and atorvastatin on lipoprotein subclasses in patients with Type 2 diabetes mellitus. Diabet Med. 2011 Jan 18. DOI:10.1111/j.1464-5491.2011.03240.x. [Epub ahead of print].

458. Rosenson RS, Otvos JD, Hsia J. Effects of rosuvastatin and atorvastatin on low-density and high-density lipoprotein particle concentrations in patients with the metabolic syndrome: a randomized, double-blind, controlled study. Diabetes Care. 2009;32(6):1087-1091.

459. Dayspring T, Dall T, Abuhajir M. Moving beyond LDL-C: incorporating lipoprotein particle numbers and geometric parameters to improve clinical outcomes. Research Reports Clin Cardiol. 2010;1: 1-10.

460. Liu J, Sempos CT, Donahue RP, Dorn J, Trevisan M, Grundy SM. Nonhigh-density lipoprotein and very-low-density lipoprotein cholesterol and their risk predictive values in coronary heart disease. Am J Cardiol. 2006;98(10):1363-1368.
461. Takahashi O, Glasziou PP, Perera R, et al. Lipid re-screening: what is the best measure and interval? Heart. 2010;96(6):448-452.

462. Glasziou PP, Irwig L, Heritier S, et al. Monitoring cholesterol levels: measurement error or true change? Ann Intern Med. 2008;148(9): 656-661.

463. McQueen MJ, Hawken S, Wang X, et al. Lipids, lipoproteins, and apolipoproteins as risk markers of myocardial infarction in 52 countries (the INTERHEART study): a case-control study. Lancet. 2008;372(9634):224-233.

464. Nordestgaard BG, Chapman MJ, Ray K, et al; for the European Atherosclerosis Society Consensus Panel. Lipoprotein(a) as a cardiovascular risk factor: current status. Eur Heart J. 2010;31(23): 2844-2853.

465. Barter PJ, Ballantyne CM, Carmena R, et al. Apo B versus cholesterol in estimating cardiovascular risk and in guiding therapy: report of the thirty-person/ten country panel. J Intern Med. 2006;259(3): 247-258.

466. Cromwell WC, Otvos JD, Keyes MJ, et al. LDL particle number and risk of future cardiovascular disease in the Framingham offspring study - implications for LDL management. J Clin Lipidol. 2007; 1(6):583-592.

467. Libby P. Inflammation in atherosclerosis. Nature. 2002;420(6917): 868-874.

468. Blake GJ, Ridker PM. Inflammatory bio-markers and cardiovascular risk prediction. J Intern Med. 2002;252(4):283-294.

469. Esposito K, Giugliano D. Diet and inflammation: a link to metabolic and cardiovascular diseases. Eur Heart J. 2006;27(1):15-20.

470. Ross R. The pathogenesis of atherosclerosis-a perspective for the 1990s. Nature. 1993;362(6423):801-809.

471. Libby P, Okamoto Y, Rocha VZ, Folco E. Inflammation in atherosclerosis: transition from theory to practice. Circ J. 2010;74(2): 213-220.

472. Hansson GK, Libby P, Schönbeck U, Yan ZQ. Innate and adaptive immunity in the pathogenesis of atherosclerosis. Circ Res. 2002; 91(4):281-291.

473. Hansson GK. Inflammation, atherosclerosis, and coronary artery disease. N Engl J Med. 2005;352(16):1685-95.

474. Libby P, Nahrendorf M, Pittet MJ, Swirski FK. Diversity of denizens of the atherosclerotic plaque: not all monocytes are created equal. Circulation. 2008;117(25):3168-3170.

475. Selvin E, Paynter NP, Erlinger TP. The effect of weight loss on c-reactive protein: a systematic review. Arch Intern Med. 2007;167(1):31-39.

476. Koenig W, Sund M, Frohlich M, et al. C-reactive protein, a sensitive marker of inflammation, predicts future risk of coronary heart disease in initially healthy middle-aged men: results from the MONICA (Monitoring Trends and Determinants in Cardiovascular Disease) Augsburg Cohort Study, 1984 to 1992. Circulation. 1999;(2):237-242.

477. Ridker PM, Buring JE, Cook NR, Rifai N. C-Reactive protein, the metabolic syndrome, and risk of incident cardiovascular events. An 8-year follow-up of 14719 initially healthy American women. Circulation. 2003;107(3):391-397.

478. Jialal I. The role of the laboratory in the diagnosis of the metabolic syndrome. Am J Clin Pathol. 2009;132(2):161-162.

479. Pitsavos C, Panagiotakos DB, Tzima N, et al. Diet, exercise, and C-reactive protein levels in people with abdominal obesity: the ATTICA Epidemiological Study. Angiology. 2007;58(2):225-233.

480. Esmaillzadah A, Kimiagar M, Mehrabi Y, Azadbakht L, Hu FB, Willett WC. Fruit and vegetable intakes, C-reactive protein, and the metabolic syndrome. Am J Clin Nutr. 2006;84(6):1489-2497.

481. Micallef MA, Munro IA, Garg ML. An inverse relationship between plasma n-3 fatty acids and C-reactive protein in healthy individuals. Eur J Clin Nutr. 2009;63(9):1154-1156.

482. Imhof A, Froehlich M, Brenner H, et al. Effect of alcohol consumption on systemic markers of inflammation. Lancet. 2001;357(9258):763-767.

483. Albert MA, Glynn RJ, Ridker PM. Alcohol consumption and plasma concentration of C-reactive protein. Circulation. 2003;107(3): $443-447$. 
484. Greenfield JR, Samaras K, Jenkins AB, et al. Obesity is an important determinant of baseline serum $\mathrm{C}$-reactive protein concentration in monozygotic twins, independent of genetic influences. Circulation. 2004;109(24):3022-3028.

485. Jiao P, Xu H. Adipose inflammation: cause or consequence of obesity-related insulin resistance. Diabetes Metab Syndr Obes. 2008;1:25-31.

486. Schuster DP. Obesity and the development of type 2 diabetes: the effects of fatty tissue inflammation. Diabetes Metab Syndr Obes. 2010;3:253-262.

487. Wang A, Nakayama T. Inflammation, a link between obesity and cardiovascular disease. Mediators Inflamm. 2010:535918.

488. Lin $\mathrm{Xu} \mathrm{L}$, Jiang CQ, Lam $\mathrm{TH}$, et al. The metabolic syndrome is associated with subclinical atherosclerosis independent of insulin resistance: the Guangzhou Biobank Cohort Study-CVD. Clin Endocrinol (Oxf). 2010;73(2):181-188.

489. Esposito K, Pontillo A, Di Palo C, et al. Effect of weight loss and lifestyle changes on vascular inflammatory markers in obese women: a randomized trial. JAMA. 2003;289(14):1799-1804.

490. Garg R, Tripathy D, Dandona P. Insulin resistance as a proinflammatory state: mechanisms, mediators, and therapeutic interventions. Curr Drug Targets. 2003;4(6):487-492.

491. Esposito K, Marfella R, Ciotola M, et al. Effect of a Mediterraneanstyle diet on endothelial dysfunction and markers of vascular inflammation in the metabolic syndrome: a randomized trial. JAMA. 2004;292(12):1440-1446.

492. Belalcazar LM, Reboussin DM, Haffner SM, et al; for The Look AHEAD Research Group. A 1-year lifestyle intervention for weight loss in individuals with type 2 diabetes reduces high C-reactive protein levels and identifies metabolic predictors of change. Diabetes Care. 2010;33(11):2297-2303.

493. Wassel CL, Barrett-Connor E, and Laughlin G. Association of circulating C-reactive protein and interleukin-6 with longevity into the 80s and 90s: The Rancho Bernardo Study. J Clin Endocrinol Metab. 2010;95(10):4748-4755.

494. Kravitz BA, Corrada MM, Kawas CH. High levels of serum C-reactive protein are associated with greater risk of all-cause mortality, but not dementia, in the oldest-old: results from the 90+ Study. JAm Ger Soc. 2009;57(4):641-646.

495. Currie CJ, Poole CD, Conway P. Evaluation of the association between the first observation and the longitudinal change in $\mathrm{C}$-reactive protein, and all-cause mortality. Heart. 2008;94(4):457-462.

496. Marsik C, Kazemi-Shirazi L, Schickbauer T, et al. C-reactive protein and all-cause mortality in a large hospital-based cohort. Clin Chem. 2008;54(2):343-349.

497. Kaptoge S, Di Angelantonio E, Lowe G, et al. The Emerging Risk Factors Collaboration. C-reactive protein concentration and risk of coronary heart disease, stroke, and mortality: an individual participant meta-analysis. Lancet. 2010;375(9709):132-140.

498. Kangasniemi O-P, Biancari F, Luukkonen J, et al. Preoperative $\mathrm{C}$-reactive protein is predictive of long-term outcome after coronary artery bypass surgery. Eur J Cardiothorac Surg. 2006;29(6): 983-985.

499. Ishii H, Toriyama T, Aoyama T, et al. Prognostic values of C-reactive protein levels on clinical outcome after implantation of sirolimuseluting stents in patients on hemodialysis. Circ Cardiovasc Interv. 2009;2(6):513-518.

500. Otake H, Shite J, Shinke T, et al. Relation between plasma adiponectin, high sensitivity C-reactive protein, and coronary plaque components in patients with acute coronary syndrome. Am J Cardiol. 2008; 101(1):1-7.

501. Morrow DA, de Lemos JA, Sabatine MS, et al. Clinical relevance of C-reactive protein during follow-up of patients with acute coronary syndromes in the Aggrastat-to-Zocor Trial. Circulation. 2006;114(4):281-288.

502. Nissen SE. Does intensive statin therapy lower mortality and cardiovascular event risk in patients with acute coronary syndrome? Nat Clin Pract Cardiovasc Med. 2005;2(1):10-11.
503. Radjesh J, Bisoendial RJ, Boekholdt SM, Vergeer M, Stroes ESG, Kastelein JJP. C-reactive protein is a mediator of cardiovascular disease. Eur Heart J. 2010;31(17):2087-2091.

504. Weber M, Bhatt DL, Brennan DM, et al; for the CHARISMA Investigators. High-sensitivity $\mathrm{C}$-reactive protein and clopidogrel treatment in patients at high risk of cardiovascular events: a substudy from the CHARISMA trial. Heart. 2011;97(8):626-631.

505. Hansson GK, Libby P. The immune response in atherosclerosis: a double-edged sword. Nat Rev Immunol. 2006;6(7):508-519.

506. Packard RR, Libby P. Inflammation in atherosclerosis: from vascular biology to biomarker discovery and risk prediction. Clin Chem. 2008; 54(1):24-38.

507. Takeuchi O, Akira S. Pattern recognition receptors and inflammation. Cell. 2010;140(6):805-820.

508. Chang M-K, Binder CH, Torzewski M, Witztum JL. C-reactive protein binds to both oxidized LDL and apoptotic cells through recognition of a common ligand: Phosphorylcholine of oxidized phospholipids. Proc Natl Acad Sci U S A. 2002;99(20):13043-13048.

509. Bu DX, Lichtman AH. T cells and blood vessels. costimulation turns up the pressure. Circulation. 2010;122(24):2495-2498.

510. Lumeng CN, Maillard I, Saltiel AR. T-ing up inflammation in fat. Nat Med. 2009;15(8):846-847.

511. Vinh A, Chen W, Blinder Y, et al. Inhibition and genetic ablation of the B7/CD28 T-cell costimulation axis prevents experimental hypertension. Circulation. 2010;122(24):2529-2537.

512. Rizzo M, Corrado E, Coppola G, et al. The predictive role of C-reactive protein in patients with hypertension and subclinical atherosclerosis. Intern Med J. 2009;39(8):539-545.

513. Novo S, Russo R, Toia P, et al. Subclinical atherosclerosis, inflammation and events. Journal ESC Council Clin Cardiol. 2011;19(16): $1-3$.

514. Van Royen N, Piek JJ, Schaper W, Fulton WF. A critical review of clinical arteriogenesis research. J Am Coll Cardiol. 2009;55(1):17-25.

515. De Groot D, Hoefer IE, Grundmann S, et al. Arteriogenesis requires toll-like receptor 2 and 4 expression in bone-marrow derived cells. J Mol Cell Cardiol. 2011;50(1):25-32.

516. Monaco C. Innate immunity meets arteriogenesis: The versatility of toll-like receptors. J Mol Cell Cardiol. 2011;50(1):9-12.

517. De Lorgeril M, Salen P, Martin JL, Monjaud I, Boucher P, Mamelle N. Mediterranean dietary pattern in the randomized trial: prolonged survival and possible reduced cancer rate. Arch Int Med. 1998;158(11): 1181-1187.

518. De Lorgeril M, Salen P, Martin JL, Monjaud I, Delaye J, Mamelle N. Mediterranean diet, traditional risk factors, and the rate of cardiovascular complications after myocardial infarction - final report of the Lyon Diet Heart Study. Circulation. 1999;99(6):779-785.

519. Stamler J, Stamler R, Neaton JD, et al. Low risk-factor profile and long-term cardiovascular and noncardiovascular mortality and life expectancy: findings for 5 large cohorts of young adult and middleaged men and women. JAMA. 1999;282(21):2012-2018.

520. De Lorgeril M, Salen P. Diet as preventive medicine in cardiology. Curr Opin Cardiol. 2000;15(5):364-370.

521. Knoops KT, de Groot LC, Kromhout D, et al. Mediterranean diet, lifestyle factors, and 10-year mortality in elderly European men and women: the HALE project. JAMA. 2004;292(12):1433-1439.

522. Trichopoulou A, Costacou T, Bamia C, Trichopoulos D. Adherence to a Mediterranean diet and survival in a Greek population. $N$ Engl $J$ Med. 2003;348(26):2599-2608.

523. Mitrou PN, Kipnis V, Thiébaut ACM, et al. Mediterranean dietary pattern and prediction of all-cause mortality in a US population: results from the NIH-AARP Diet and Health Study. Arch Intern Med. 2007; 167(22):2461-2468.

524. Crowe FL, Roddam AW, Key TJ, and European Prospective Investigation into Cancer and Nutrition (EPIC)-Heart Study Collaborators. Fruit and vegetable intake and mortality from ischaemic heart disease: results from the European Prospective Investigation into Cancer and Nutrition (EPIC)-Heart study. Eur Heart J. 2011;32(10): 1235-1243. 
525. Capewell S, Lloyd-Jones DM. Optimal cardiovascular prevention strategies for the 21st century. JAMA. 2010;304(18):2057-2058.

526. Wilson JT, Lloyd-Jones DM. Biomarkers for coronary heart disease clinical risk prediction: A critical appraisal. Prev Cardiol. 2010;13(4):160-165.

527. Robinson JG, Wang S, Smith BJ, Jacobson TA. Meta-analysis of the relationship between non-high-density lipoprotein cholesterol reduction and coronary heart disease risk. J Am Coll Cardiol. 2009; 53(4):316-322.

528. Blaha M, Blumenthal R, Brinton E, Jacobson T, On behalf of the National Lipid Association Taskforce on Non-HDL Cholesterol. The importance of non-HDL cholesterol reporting in lipid management. J Clin Lipidol. 2008;2(4):267-273.

529. Robinson JG. Are you targeting non-high-density lipoprotein cholesterol? J Am Coll Cardiol. 2010;55(1):42-44.

530. Wilkins JT, Lloyd-Jones DM. Short-term versus lifetime risk assessment for cardiovascular disease: Pros, cons, and clinical implications. Curr Cardiovasc Risk Rep. 2009;3(2):144-149.

531. Pencina MJ, D’Agostino RB Sr, Larson MG, Massaro JM, Vasan RS. Predicting the 30-year risk of cardiovascular disease: the Framingham Heart Study. Circulation. 2009;119(24):3078-3084.

532. Ridker PM, Danielson E, Fonseca FA, et al; JUPITER Study Group. Rosuvastatin to prevent vascular events in men and women with elevated C-reactive protein. N Engl J Med. 2008;359(21):2195-2207.

533. Mancia G, Laurent S, Agabiti-Rosei E, et al. Reappraisal of European guidelines on hypertension management: a European Society of Hypertension Task Force document. J Hypertension. 2009;27(11):2121-2158.

534. Koenig W, Ridker PM. Rosuvastatin for primary prevention in patients with European systematic coronary risk evaluation risk $>5 \%$ or Framingham risk $>20 \%$ : Post hoc analyses of the JUPITER trial requested by the European health authorities. Eur Heart J. 2011; 32(1):75-83.

535. Choudhry NK, Patrick AR, Glynn RJ, Avorn J. The cost-effectiveness of C-reactive protein testing and rosuvastatin treatment for patients with normal cholesterol levels. J Am Coll Cardiol. 2011;57(7):784-791.

536. Hlatky M. The cost-effectiveness of rosuvastatin therapy: JUPITER (Justification for the Use of statins in Prevention: An Intervention Trial Evaluating Rosuvastatin). J Am Coll Cardiol. 2011;57(7): 792-793.

537. De Lorgeril M, Salen P, Abramson J; et al. Cholesterol lowering, cardiovascular diseases, and the rosuvastatin-JUPITER controversy: a critical reappraisal. Arch Intern Med. 2010;170(12):1032-1036.

538. Carrera-Bastos P, Fontes M, O'Keefe JH, Lindeberg S, Cordain L. The Western Diet and lifestyle and diseases of civilization. Res Reports Clin Cardiol. 2011;2:15-35.

539. Mookadam F, Moustafa SE, Lester SJ, Warsame T. Subclinical atherosclerosis: Evolving role of carotid intima-media thickness. Prev Cardiol. 2010;13(4):186-197.

540. Liviakis L, Pogue B, Paramsothy P, Bourne A, Gill EA. Carotid intima-media thickness for the practicing lipidologist. J Clin Lipidol. 2010;4(1):24-35.

541. Goldberger ZD, Valle JA, Dandekar VK, et al. Are changes in carotid intima-media thickness related to risk of nonfatal myocardial infarction? A critical review and meta-regression analysis. Amer Heart J. 2010;160(4):701-714.

542. Nambi V, Chambless L, Folsom AR, et al. Carotid intima-media thickness and presence or absence of plaque improves prediction of coronary heart disease risk: the ARIC (Atherosclerosis Risk In Communities) study. J Am Coll Cardiol. 2010;55(15):1600-1607.

543. Gerber TC, Taylor AJ. Carotid intima-media thickness: can it close the "detection gap" for cardiovascular risk? Mayo Clin Proc. 2009; 84(3):218-220.

544. Lester SJ, Eleid MF, Khandheria BK, Hurst RT. Carotid intima-media thickness and coronary artery calcium score as indications of subclinical atherosclerosis. Mayo Clin Proc. 2009;84(3):229-233.

545. Stein JH, Johnson HM. Carotid intima-media thickness, plaques, and cardiovascular disease risk: implications for preventive cardiology guidelines. J Am Coll Cardiol. 2010;55(15):1608-1610.
546. Sibal L, Agarwal SC, Home PD. Carotid intima-media thickness as a surrogate marker of cardiovascular disease in diabetes. Diab Metab Syndr Obesity. 2011:4:23-34.

547. Sharma K, Blaha MJ, Blumenthal RS, Musunuru K. Clinical and Research applications of carotid intima-media thickness. Am J Cardiol. 2009;103(9):1316-1320.

548. Costanzo P, Perrone-Filardi P, Vassallo E, et al. Does carotid intimamedia thickness regression predict reduction of cardiovascular events? A meta-analysis of 41 randomized trials. J Am Coll Cardiol. 2010;56(24):2006-2020.

549. Stein JH, Korcarz CE, Hurst RT, et al. Use of carotid ultrasound to identify subclinical vascular disease and evaluate cardiovascular disease risk: a consensus statement from the American Society of Echocardiography Carotid Intima-Media Thickness Task Force. J Am Soc Echocardiogr. 2008;21(2):93-111.

550. United States Preventive Services Task Force. Using nontraditional risk factors in coronary heart disease risk assessment: US Preventive Services Task Force Recommendation Statement. Ann Intern Med. 2009;151(7):474-482.

551. Polonsky TS, McClelland RL, Jorgensen NW, et al. Coronary artery calcium score and risk classification for coronary heart disease prediction. JAMA. 2010;303(16):1610-1616.

552. Detrano R, Guerci AD, Carr JJ, et al. Coronary calcium as a predictor of coronary events in four racial or ethnic groups. N Engl J Med. 2008; 358(13):1336-1345.

553. Shah PK. The SHAPE Paradigm: a commentary. Circ Cardiovasc Qual Outcomes. 2010;3(1):106-109.

554. Patel A, Budoff M. Screening for heart disease: C-reactive protein versus coronary artery calcium. Expert Rev Cardiovasc Ther. 2010; 8(1):125-131.

555. Erbel R, Möhlenkamp S, Moebus S, et al; for the Heinz Nixdorf Recall Study Investigative Group. Coronary risk stratification, discrimination, and reclassification improvement based on quantification of subclinical coronary atherosclerosis: The Heinz Nixdorf Recall Study. J Am Coll Cardiol. 2010;56(17):1397-1406.

556. Elias-Smale SE, Proença RV, Koller MT, et al. Coronary calcium score improves classification of coronary heart disease risk in the elderly: The Rotterdam Study. J Am Coll Cardiol. 2010;56(17):1407-1414.

557. Duprez D. Coronary artery calcification, an improvement in risk classification: need for reappraisal? J Am Coll Cardiol. 2010;56(17): 1415-1417.

558. Rumberger JA. Using noncontrast cardiac CT and coronary artery calcification measurements for cardiovascular risk assessment and management in asymptomatic adults. Vasc Health Risk Manag. 2010; 6:579-591.

559. Piers LH, Salachova F, Slart RHJA, et al. The role of coronary artery calcification score in clinical practice. BMC Cardiovascular Disorders. 2008;8:38.

560. Raggi P, Gongora MC, Gopal A, Callister TQ, Budoff M, Shaw LJ. Coronary artery calcium to predict all-cause mortality in elderly men and women. J Am Coll Cardiol. 2008;52(1):17-23.

561. Sharma RK, Sharma RK, Voelker DJ, et al. Cardiac risk stratification: role of the coronary calcium score. Vasc Health Risk Manag. 2010;6:603-611.

562. Naghavi M, Falk E, Hecht HS, Shah PK, For the SHAPE Task Force. The First SHAPE (Screening for Heart Attack Prevention and Education) Guideline. Crit Pathw Cardiol. 2006;5(4):187-190.

563. Naghavi M. Screening for early detection and prevention of heart attack. Presented at the American College of Cardiology 59th Annual Scientific Session, March, 2010. Available at: www.shapesociety.org/ images/downloadshapepresentation.jpg. Accessed March 24, 2011.

564. Naghavi M, editor. Asymptomatic Atherosclerosis: Pathophysiology, Detection and Treatment. New York: Humana Press/Springer-Verlag; 2011. ISBN 978-1-60327-178-3.

565. Nasir K, Michos ED, Blumenthal RS, Raggi P. Detection of highrisk young adults and women by coronary calcium and National Cholesterol Education Program Panel III Guidelines. J Am Coll Cardiol. 2005;46(10):1931-1936. 
566. Shaw LJ, Raggi P, Berman DS, Callister TQ. Coronary artery calcium as a measure of biologic age. Atherosclerosis. 2006;188(1):112-119.

567. Harvey S, Hecht HS. A zero coronary artery calcium score: priceless. J Am Coll Cardiol. 2010;55(11):1118-1120.

568. Kabul P, Douglas PS. Atherosclerosis imaging: prognostically useful or merely more of what we know? Circ Cardiovasc Imaging. 2009; 2(2):150-160.

569. Richard Conti C. Is calcium scoring of the coronary arteries necessary for proper management of asymptomatic subjects with classic risk factors? Clin Cardiol. 2010;33(11):658-659.

570. Budoff MJ, Gupta M. Radiation exposure from cardiac imaging procedures: do the risks outweight the benefits? $J$ Am Coll Cardiol. 56(9):712-714

571. Chen J, Einstein AJ, Fazel R, et al. cumulative exposure to ionizing radiation from diagnostic and therapeutic cardiac imaging procedures: a population-based analysis. $\mathrm{J} \mathrm{Am} \mathrm{Coll} \mathrm{Cardiol}$. 2010;56(9):702-711.

572. Gibbons RJ, Gerber TC. Calcium scoreing with computed tomography: what is the radiation risk? Arch Int Med. 2009;169(13):1185-1187.

573. Mccullough PA, Chinnaiyan KM. Annual progression of coronary calcification in trials of preventive therapies: a systematic review. Arch Int Med. 2009;169(22):2064-2070.

574. O'Malley PG. A double take on serial measurement of coronary artery calcification. Arch Int Med. 2009;169(22):2051-2052.

575. See R, Lindsey JB, Patel MJ, et al. Application of the screening for Heart Attack Prevention and Education Task Force Recommendations to an urban population: observations from the Dallas Heart Study. Arch Intern Med. 2008;168(10):1055-1062.

576. Pletcher MJ, Greenland P. Coronary calcium scoring and cardiovascular risk: the SHAPE of things to come. Arch Intern Med. 2008;168(10):1027-1028.

577. Ioannidis JPA, Tzoulaki I. What makes a good predictor? the evidence applied to coronary artery calcium score. JAMA. 2010;303(16):1646-1647.

578. Einstein A, Weiner S, Bernheim A, et al. Multiple testing, cumulative radiation dose, and clinical indications in patients undergoing myocardial perfusion imaging. JAMA. 2010;304(19):2137-2144.

579. Eisenberg MJ, Afilalo JA, Lawler PR, Abrahamowicz M, Richard H, Pilote L. Cancer risk related to low-dose ionizing radiation from cardiac imaging in patients after acute myocardial infarction. CMAJ. 2011;182(4):430-436.

580. Dorfman AL, Fazel R, Einstein AJ, et al. Use of medical imaging procedures with ionizing radiation in children: a population-based study. Arch Pediatr Adolesc Med. 2011;165(5):458-464.

581. Fazel R, Krumholz HM, Wang Y, et al. Exposure to low-dose ionizing radiation from medical imaging procedures. $N$ Engl $J$ Med. 2009;361(9):849-857.

582. Sodickson A, Baeyens PF, Andriole KP, et al. Recurrent CT, cumulative radiation exposure, and associated radiation-induced cancer risks from CT of adults. Radiology. 2009;251(1):175-184.

583. Mark DB, Berman DS, Budoff MJ, et al. ACCF/ACR/AHA/NASCI/ SAIP/SCAI/SCCT 2010. Expert consensus document of coronary computed tomographic angiography: a report of the American College of Cardiology Foundation task force on expert consensus documents. $J$ Am Coll Cardiol. 2010;55(23):2663-2699.

584. Sarwar A, Shaw LJ, Shapiro MD, et al. diagnostic and prognostic value of absence of coronary artery calcification. JACC Cardiovasc Imaging. 2009;2(6):675-688.

585. Garcia MJ, Fuster V. An ounce of prevention with a calcium score scan? JACC Cardiovasc Imaging. 2009;2(6):689-691.

586. Cademartiri F, Maffei E, Palumbo A, et al. Coronary calcium score and computed tomography coronary angiography in high-risk asymptomatic subjects: assessment of diagnostic accuracy and prevalence of nonobstructive coronary artery disease. Eur Radiol. 2010;20(4):846-854.

587. Gottlieb I, Miller JM, Arbab-Zadeh A, et al. The absence of coronary calcification does not exclude obstructive coronary artery disease or the need for revascularization in patients referred for conventional coronary angiography. J Am Coll Cardiol. 2010;55(7):627-634.
588. Redberg RF. What is the prognostic value of a zero calcium score? J Am Coll Cardiol. 2010;55(7):635-636.

589. Baumann BM, Chen EH, Mills AM, et al. Patient perceptions of computed tomographic imaging and their understanding of radiation risk and exposure. Ann Emerg Med. 2010 Dec 10. [Epub ahead of print].

590. Berrington de Gonzalez A, Mahesh M, Kim KP, et al. Projected cancer risks from computed tomographic scans performed in the United States in 2007. Arch Intern Med. 2009;169(22):2071-2077.

591. Fernandez-Friera L, Garcia-Alvarez A, Bagheriannejad-Esfahani F, et al. diagnostic value of coronary artery calcium scoring in lowintermediate risk patients evaluated in the emergency department for acute coronary syndrome. Am J Cardiol. 2011;107(1):17-23.

592. Nabi F, Chang SM, Pratt CM, et al. Coronary artery calcium scoring in the emergency department: identifying which patients with chest pain can be safely discharged home. Ann Emerg Med. 2010; 56(3):220-229.

593. Budoff MJ. Coronary calcium remains an effective filter for invasive angiography. J Am Coll Cardiol. 2010;56(7):613-614.

594. Orringer CE. The absence of coronary calcium: clinical and therapeutic implications for the clinical lipidologist. J Clin Lipidol. 2010;4(6): 472-477.

595. Redberg RF. Reply. J Am Coll Cardiol. 2010;56(7):615-615.

596. McEvoy JW, Blaha MJ, DeFilippis AP, et al. Coronary artery calcium progression: an important clinical measurement? A review of published reports. J Am Coll Cardiol. 2010;56(20):1613-1622.

597. Heart Protection Study Collaborative Group. Statin cost-effectiveness in the United States for people at different vascular risk levels. Circ Cardiovasc Qual Outcomes. 2009;2(2):65-72.

598. Hayward RA, Krumholz HM, Zulman DM, Timbie JW, Vijan S. Optimizing statin treatment for primary prevention of coronary artery disease. Ann Intern Med. 2010;152(2):69-77.

599. Ridker PM, Rifai N, Clearfield M, et al. Measurement of C-reactive protein for the targeting of statin therapy in the primary prevention of acute coronary events. $N$ Engl J Med. 2001;344(26): 1959-1965.

600. Mahoney EM. Targeting patients for statin therapy for the primary prevention of vascular events: what is the best approach? Circulation. 2010;122(15):1446-1448.

601. Hayward RA, Hofer TP, Vijan S. Narrative review: lack of evidence for recommended low-density lipoprotein treatment targets: a solvable problem. Ann Intern Med. 2006;145(7):520-530.

602. Li M, Ong KL, Tse HF, Cheung BM. Utilization of lipid lowering medications among adults in the United States 1999-2006. Atherosclerosis. 2010;208(2):456-460.

603. Lewis LS. Strategies for statins: The CASE for fire and forget. $B M J$ 2008;336(7641):406.

604. Lewis LS. The most cost-effective statin strategy? BMJ. 2010;341:c3531. Available at: http://www.bmj.com/content/341/bmj.c3531/reply.

605. Sharma M, Ansari MT, Abou-Setta AM, et al. Systematic review: comparative effectiveness and harms of combination therapy and monotherapy for dyslipidemia. Ann Int Med. 2009;151(9):622-630.

606. Krumholz HM, Hayward RA. Shifting views on lipid lowering therapy. BMJ. 2010;341:c3531.

607. Knott L. Patient UK. Primary prevention of cardiovascular disease. Updated June 28, 2010. Available at: http://www.patient.co.uk/doctor/ Primary-Prevention-of-Cardiovascular-Disease-(CVD).htm. Accessed March 24, 2011.

608. O’Keefe Jr JH, Cordain L, Harris WH, Moe RM, Vogel R. Optimal low-density lipoprotein is 50 to $70 \mathrm{mg} / \mathrm{dl}$ : lower is better and physiologically normal. J Am Coll Cardiol. 2004;43(11):2142-2146.

609. Hochholzer W, Giugliano RP. Lipid lowering goals: back to nature? Ther Adv Cardiovasc Dis. 2010;4(3):185-191.

610. O'Keefe Jr H, Cordain L. Cardiovascular disease resulting from a diet and life-style at odds with our Paleolithic genome: how to become a 21st-century hunter-gatherer. Mayo Clin Proc. 2004;79(1): 101-108. 
611. Chen Z, Peto R, Collins R, MacMahon S, Lu J, Li W. Serum cholesterol concentration and coronary heart disease in population with low cholesterol concentrations. BMJ. 1991;303(6797):276-282.

612. Dietschy JM, Turley SD. thematic review series: brain lipids. Cholesterol metabolism in the central nervous system during early development and in the mature animal. J Lipid Res. 2004;45(8): 1375-1397.

613. Drexel H, Aczel S, Marte T, Vonbank A, Saely CH. Factors predicting cardiovascular events in statin-treated diabetic and non-diabetic patients with coronary atherosclerosis. Atherosclerosis. 2010; 208(2):484-489.

614. Wald NJ, Law MR. A strategy to reduce cardiovascular disease by more than 80\%. BMJ. 2003;326(7404):1419.

615. Yusuf S, Pais P, Afzal R, et al; the Indian Polycap Study (TIPS). Effects of a polypill (Polycap) on risk factors in middle-aged individuals without cardiovascular disease (TIPS): a phase II, double-blind, randomised trial. Lancet. 2009;373(9672):1341-1351.

616. Cannon CP. Can the polypill save the world from heart disease? Lancet. 2009;373(9672):1313-1314.

617. Soliman EZ, Mendis S, Dissanayake WP, et al. A Polypill for primary prevention of cardiovascular disease: A feasibility study of the World Health Organization. Trials. 2011;12:3.

618. Fuster V, Sanz G. A polypill for secondary prevention: time to move from intellectual debate to action. Nat Clin Pract Cardiovasc Med. 2007;4(4):173.

619. Fuster V. Prime time for a polypill after myocardial infarction? Nat Clin Pract Cardiovasc Med. 2009;6(2):83.

620. Wald NJ, Wald DS. The polypill concept. Heart. 2010;96(1):1-4.

621. Maciosek MV, Coffield AB, Flottemesch TJ, Edwards NM, and Solberg LI. Greater Use Of Preventive Services In US Health Care Could Save Lives At Little Or No Cost. Health Affairs. 2010;29(9): 1656-1660.

622. Webster R, Heeley E. Perceptions of risk: understanding cardiovascular disease. Risk Manag Healthc Policy. 2010;3:49-60.

623. Mosca L, Mochari-Greenberger H, Dolor RJ, Newby LK, Robb KJ. Twelve-year follow-up of American women's awareness of cardiovascular disease risk and barriers to heart health. Circ Cardiovasc Qual Outcomes. 2010;3(2):120-127.

624. Pancioli AM, Broderick J, Kothari R, et al. Public perception of stroke warning signs and knowledge of potential risk factors. JAMA. 1998;279(16):1288-1292.

625. Ritchie JE, Herscovitch F, Norfor JB. Beliefs of blue collar workers regarding coronary risk behaviours. Health Educ Res. 1994;9(1): 95-103.
626. Norcross JC, Krebs PM, Prochaska JO. Stages of change. J Clin Psychol. 2011;67(2):143-154.

627. Janz NK, Becker MH. The Health Belief Model: a decade later. Health Educ Q. 1984;11(1):1-47.

628. Weinstein ND. Unrealistic optimism about susceptibility to health problems: conclusions from a community-wide sample. J Behav Med. 1987;10(5):481-500.

629. Mosca L, Benjamin EJ, Berra K, et al. Effectiveness-based guidelines for the prevention of cardiovascular disease in women - 2011 update. A guideline from the American Heart Association. Circulation. 2011; 123(11):1243-1262.

630. Robins SJ, Lyass A, Zachariah JP, Massaro JM, Vasan RS. Insulin resistance and the relationship of a dyslipidemia to coronary heart disease: the Framingham Heart Study. Arterioscler Thromb Vasc Biol. 2011;31(5):1208-1214.

631. Möhlenkamp S, Lehmann N, Moebus S, et al; for the Heinz Nixdorf Recall Study Investigators. Quantification of coronary atherosclerosis and inflammation to predict coronary events and all-cause mortality. J Am Coll Cardiol. 2011;57(13):1455-1464.

632. Hamm CW, Nef HM, Rolf A, Möllmann H. Calcium and C-reactive protein: hot enough to predict the future? J Am Coll Cardiol. 2011; 57(13):1465-1467.

633. Chironi G, Simon A, Megnien J-L, et al. Coronary calcium data have limited impact on CHD risk-classification. Int J Cardiol. 2010; advance publication. DOI: 10.1016/j.ijcard.2010.05.024. In press.

634. Rubin J, Chang H-J, Nasir K, et al. Association between high-sensitivity C-reactive protein and coronary plaque subtypes assessed by 64-slice coronary computed tomography angiography in an asymptomatic population. Circ Cardiovasc Imaging. 2011:4:201-209.

635. Ridker PM. High-sensitivity C-reactive protein, vascular imaging, and vulnerable plaque. More evidence to support trials of antiinflammatory therapy for cardiovascular risk reduction. Circ Cardiovasc Imaging. 2011;4:195-197.

636. deGoma EM, Rader DJ. Novel HDL-directed pharmacotherapeutic strategies. Nat Rev Cardiol. 2011;8:266-277.

637. Bruckert E, Labreuche J, Amarenco P. Meta-analysis of the effect of nicotinic acid alone or in combination on cardiovascular events and atherosclerosis. Atherosclerosis. 2010;210(2):353-361.
Drug Design, Development and Therapy

\section{Publish your work in this journal}

Drug Design, Development and Therapy is an international, peerreviewed open-access journal that spans the spectrum of drug design and development through to clinical applications. Clinical outcomes, patient safety, and programs for the development and effective, safe, and sustained use of medicines are a feature of the journal, which

\section{Dovepress}

has also been accepted for indexing on PubMed Central. The manuscript management system is completely online and includes a very quick and fair peer-review system, which is all easy to use. Visit http://www.dovepress.com/testimonials.php to read real quotes from published authors. 